BULLETIN (New Series) OF THE

AMERICAN MATHEMATICAL SOCIETY

Volume 39, Number 4, Pages 475-531

S 0273-0979(02)00951-5

Article electronically published on July 8, 2002

\title{
A CENTURY OF COMPLEX TAUBERIAN THEORY
}

\author{
J. KOREVAAR
}

\begin{abstract}
Complex-analytic and related boundary properties of transforms give information on the behavior of pre-images. The transforms may be power series, Dirichlet series or Laplace-type integrals; the pre-images are series (of numbers) or functions.

The chief impulse for complex Tauberian theory came from number theory. The first part of the survey emphasizes methods which permit simple derivations of the prime number theorem, associated with the labels LandauWiener-Ikehara and Newman. Other important areas in complex Tauberian theory are associated with the names Fatou-Riesz and Ingham. Recent refinements have been motivated by operator theory and include local $H^{1}$ and pseudofunction boundary behavior of transforms. Complex information has also led to better remainder estimates in connection with classical Tauberian theorems. Applications include the distribution of zeros and eigenvalues.
\end{abstract}

\section{INTRODUCTION}

Tauberian theorems involve a class of objects $S$ (functions, series, sequences) and a transformation $\mathcal{T}$. The transformation is an 'averaging' operation. It must have a continuity property: certain limit behavior of the original $S$ implies related limit behavior of the image $\mathcal{T} S$. The aim of a Tauberian is to reverse the averaging: to go from a limit property of $\mathcal{T} S$ to a limit property of $S$, or another transform of $S$. The converses typically require an additional condition, a 'Tauberian condition', on $S$ and perhaps a condition on the transform $\mathcal{T} S$.

We mention some classical examples; cf. Hardy's book [1949. By Abel's theorem [1826], convergence $\sum_{0}^{\infty} a_{n}=A$, or $s_{n}=\sum_{k \leq n} a_{k} \rightarrow A$, implies that the transform

$$
f(x)=\sum a_{n} x^{n}=\frac{\sum s_{n} x^{n}}{\sum x^{n}} \text { has limit } A \text { as } x \nearrow 1 .
$$

Tauber 1897] proved that the condition $n a_{n} \rightarrow 0$ is sufficient for a converse. An optimal order condition is that $\left\{n a_{n}\right\}$ be bounded (Littlewood [1911]). Subsequently, Hardy and Littlewood proved numerous other converse theorems, and they coined the name Tauberian theorems. Tauber himself concentrated on the mathematics of insurance. Retired from his positions in Vienna, he became a victim of the Holocaust; cf. Binder [1984].

Here is another Tauberian theorem for power series $f(x)=\sum_{0}^{\infty} a_{n} x^{n}$ which converge for $|x|<1$. By a continuity theorem of Frobenius [1880, the asymptotic

Received by the editors June 28, 2001, and, in revised form, February 22, 2002.

2000 Mathematics Subject Classification. Primary 40E05; Secondary 11M45, 30B50, 44A10, $47 \mathrm{~A} 10$.

(C)2002 American Mathematical Society 
relation $s_{n}=\sum_{k \leq n} a_{k} \sim A n$ implies that

$$
f(x)=(1-x) \sum_{0}^{\infty} s_{n} x^{n} \sim \frac{A}{1-x} \quad \text { as } x \nearrow 1 .
$$

Hardy and Littlewood [1914 showed that the converse is valid under the Tauberian condition $a_{n} \geq 0$.

The situation is more delicate in the case of (classical) Dirichlet series $f^{*}(x)=$ $\sum_{1}^{\infty} a_{n} / n^{x}$, which are important in number theory. If such a series is absolutely convergent for $x>1$ and $s_{n} \sim A n$, then

$$
f^{*}(x) \sim \frac{A}{x-1} \quad \text { as } x \searrow 1
$$

However, in this case one needs much more for a converse than the condition $a_{n} \geq 0$. Here it is desirable to consider the behavior of the sum function $f^{*}$ for complex $x$.

Tauberian theorems in which complex-analytic or related boundary properties of the transform play an important role are called complex Tauberians. The first results of that kind were Tauberian theorems avant la lettre. For power series there was Fatou's theorem [1905], [1906]: If the transform $f(z)=\sum_{0}^{\infty} a_{n} z^{n}$ of the series $\sum_{0}^{\infty} a_{n}$ exists for $|z|<1$ and is analytic at the point $z=1$, then the condition $a_{n} \rightarrow 0$ already implies the convergence of $\sum_{0}^{\infty} a_{n}$. Cf. Littlewood's condition above! Marcel Riesz [1909], [1911, [1916] proved complex results for general Dirichlet series $\sum_{0}^{\infty} a_{n} e^{-\mu_{n} z}$. Both power series and Dirichlet series can be considered as special cases of Laplace-Stieltjes integrals

$$
F(z)=\mathcal{L} d S(z)=\int_{0-}^{\infty} e^{-z t} d S(t),
$$

which play an important role in Tauberian theory.

Number theoretic questions have inspired a great deal of complex Tauberian theory for Dirichlet series and Laplace transforms. The early proofs of the prime number theorem (PNT), by Hadamard [1896] and de la Vallée Poussin [1896], required analytic information about the Riemann zeta function on and to the left of the line $\operatorname{Re} z=1$. The best estimates known today for the prime counting function $\pi(u)$ still make use of such information. And, for one of the quickest proofs of just the prime number theorem:

$$
\pi(u) \sim \frac{u}{\log u} \quad \text { as } u \rightarrow \infty,
$$

Littlewood 1971] went all the way across the so-called critical strip $0<x=\operatorname{Re} z<$ 1 !

Since 1900 one has aimed for simple proofs of the PNT which use as little about the zeta function as possible. The so-called elementary proofs by Selberg [1949] and Erdős [1949] avoid the zeta function altogether, but they do not qualify as simple. Of numerous expositions of these proofs, we mention Levinson [1969] and Diamond 1982]. See also the general surveys by Lavrik [1984], Bateman and Diamond [1996], Apostol [2000], Tenenbaum and Mendès France [2000], and the book by Narkiewicz 2000.

Besides simple analytic properties, the minimal information about the zeta function required for a complex proof of the PNT is the nonvanishing of $\zeta(z)$ on the line $\operatorname{Re} z=1$. (The latter property is a consequence of the PNT; cf. Ingham [1932] or Corollary 3.2.) For his relatively simple proof, Landau [1908], [1909] had to 
know in addition that $\zeta^{\prime}(z) / \zeta(z)$ does not grow faster than a power of $z$ as $z \rightarrow \infty$ in the half-plane $\operatorname{Re} z \geq 1$; cf. Section 2. Hardy and Littlewood [1918] could relax this to an exponential order condition, but the real breakthrough came with Wiener's Tauberian theory [1928], [1932], 1933] (which we do not discuss here) and the work of his student Ikehara 1931. Applying Wiener's early [1928. Tauberian theory, Ikehara could dispense with the growth condition in the complex approach. The resulting 'Wiener-Ikehara theorem' has long provided the preferred way to the PNT. We discuss two proofs of the theorem. The traditional proof in Section 4 is based on an approximate identity, and this method will be important for us later on. The elegant newer proof by Graham and Vaaler 1981 in Section 5, which adds precision, is based on one-sided $L^{1}$ approximation by Fourier transforms of functions with bounded support.

A very attractive approach to the PNT is due to Newman [1980. He could prove a useful Tauberian theorem for Dirichlet series with bounded coefficients by simple contour integration. Substituting Laplace transforms of bounded functions, we describe Newman's way to the PNT in Sections 6 - 8; cf. Korevaar 1982 and Zagier [1997. This material is largely independent of Sections $2-5$.

In the 1930's the Wiener-Ikehara method was refined and extended by a number of authors, most notably Ingham [1935], [1936]. His work contains the Tauberians just referred to, as well as stronger one-sided versions, and includes refinements of Fatou's theorem. Sections $9-12$ cover such results, with partly new proofs.

Sections $13-15$ are devoted to newer developments in the Fatou-Riesz and Laplace transform area, which were motivated by operator theory and semigroups. In this introduction we only signal seminal papers by Katznelson and Tzafriri [1986], Allan, O'Farrell and Ransford [1987], and Arendt and Batty [1988]. Among other things, the refinements involve local $H^{1}$ and pseudofunction boundary behavior of transforms. Their development was aided by Newman's contour integration method as formulated in Section 7. For a detailed treatment of the numerous results and applications, see the book by Arendt, Batty, Hieber and Neubrander 2001].

There is a large body of Tauberian remainder theory involving conditions in the complex domain. We present notable examples where 'complex' information gives much stronger results than exclusively 'real' information. Sharp remainder estimates for the theorems of Fatou and Riesz were first discussed in Korevaar 1954b; cf. Section 17. Remainder estimates in Hardy-Littlewood theorems for power series under real conditions were obtained by Freud [1951-4] and Korevaar [1951], 1953, 1954a, 1954b; in Section 18 we discuss stronger estimates under complex conditions, due to Postnikov [1953] and Subhankulov 1960, 1964. We finally consider Tauberian results for the Stieltjes transform, due to Malliavin 1962 and Pleijel 1963, which have applications to the zero distribution of entire functions and the distribution of eigenvalues. For other aspects of remainder theory, see the books by Ganelius [1971], Postnikov [1979], and especially Subhankulov [1976, Russian].

Analyticity of transforms in a strip plays a role in certain Tauberian theorems involving regular variation; see Bingham, Goldie and Teugels [1987]. Multidimensional complex Tauberians have been treated by Vladimirov, Drozhzhinov and Zav'yalov [1986]. Several authors have added something to 'real' Hardy-Littlewood theorems by complex variable proofs. In this context we mention Delange [1952, Jurkat [1956], [1957], and Halász [1967]; cf. Landau and Gaier [1986, appendix II]. 
Acknowledgements. The author wishes to thank Harold Diamond for his comments and Jan Wiegerinck for $\mathrm{T}_{\mathrm{E}} \mathrm{Xnical}$ assistance.

\section{LANDAu-Type TAuberian For Dirichlet SeRIES}

Landau [1908, [1909, section 66] derived the prime number theorem from a Tauberian result of the following type.

Theorem 2.1. Let $f$ be given for $\operatorname{Re} z>1$ by a convergent Dirichlet series,

$$
f(z)=\sum_{n=1}^{\infty} \frac{a_{n}}{n^{z}}
$$

with $a_{n} \geq 0$ for all $n$. Suppose that for some constant $A$, the analytic function

$$
g(z)=f(z)-\frac{A}{z-1}, \quad \operatorname{Re} z>1
$$

has an analytic or just continuous extension (also called $g$ ) to the closed half-plane $\operatorname{Re} z \geq 1$. Finally, suppose that there is a constant $M$ such that

$$
g(z)=\mathcal{O}\left(|z|^{M}\right) \quad \text { for } \operatorname{Re} z \geq 1 .
$$

Then

$$
\frac{1}{n} s_{n}=\frac{1}{n} \sum_{1}^{n} a_{k} \rightarrow A \quad \text { as } n \rightarrow \infty .
$$

Proof. One may start with the formula

$$
\frac{1}{2 \pi i} \int_{\alpha-i \infty}^{\alpha+i \infty} \frac{e^{t z}}{z(z+1)} d z= \begin{cases}1-e^{-t} & \text { for } t \geq 0 \\ 0 & \text { for } t<0\end{cases}
$$

$(\alpha>0)$, which is an easy application of the residue theorem. We now set $\sum_{k<v} a_{k}=$ $s(v)$ (so that $s(v)=0$ for $v<1$ ) and take $\alpha>1, u>0$. Then by absolute convergence,

$$
\begin{gathered}
\frac{1}{2 \pi i} \int_{\alpha-i \infty}^{\alpha+i \infty} f(z) \frac{u^{z}}{z(z+1)} d z=\frac{1}{2 \pi i} \int_{\alpha-i \infty}^{\alpha+i \infty} \sum_{1}^{\infty} \frac{a_{n}}{n^{z}} \frac{u^{z}}{z(z+1)} d z \\
=\sum_{n \leq u} a_{n}(1-n / u)=\int_{0}^{u}(1-v / u) d s(v)=\frac{1}{u} \int_{0}^{u} s(v) d v .
\end{gathered}
$$

Again by the residue theorem,

$$
\frac{1}{2 \pi i} \int_{\alpha-i \infty}^{\alpha+i \infty} \frac{1}{z-1} \frac{u^{z}}{z(z+1)} d z= \begin{cases}(u-1)^{2} /(2 u) & \text { for } u \geq 1 \\ 0 & \text { for } 0<u<1 .\end{cases}
$$

Subtracting $A$ times the last formula from the preceding and multiplying by $u$, one obtains from (2.2) that

$$
\frac{1}{2 \pi i} \int_{\alpha-i \infty}^{\alpha+i \infty} g(z) \frac{u^{z+1}}{z(z+1)} d z= \begin{cases}\int_{0}^{u} s(v) d v-\frac{1}{2} A(u-1)^{2} & \text { for } u \geq 1 \\ 0 & \text { for } 0<u<1\end{cases}
$$

cf. Ingham [1932]. We now introduce the $k$-times iterated integral

$$
s^{(-k)}(u)=\int_{0}^{u} d v_{1} \int_{0}^{v_{1}} d v_{2} \cdots \int_{0}^{v_{k-1}} s\left(v_{k}\right) d v_{k} .
$$


Integrating $k-1$ times with respect to $u$ from 0 on in (2.5) and dividing by $u^{k+1}$, one finds that for $u \geq 1$ and $\alpha>1$,

$$
\frac{s^{(-k)}(u)}{u^{k+1}}-\frac{A}{(k+1) !}\left(1-\frac{1}{u}\right)^{k+1}=\frac{1}{2 \pi i} \int_{\alpha-i \infty}^{\alpha+i \infty} \frac{u^{z-1} g(z) d z}{z(z+1) \ldots(z+k)} .
$$

We finally use the hypothesis (2.3). Taking $k>M$ and invoking dominated convergence, one may let $\alpha$ go to 1 in (2.6). The resulting right-hand side with $z=1+i y$ is an absolutely convergent Fourier integral, involving the exponential $\exp (i y \log u)$. By the Riemann-Lebesgue lemma, this right-hand side will tend to 0 as $u \rightarrow \infty$. One thus obtains the asymptotic relation

$$
s^{(-k)}(u) \sim \frac{A}{(k+1) !} u^{k+1} .
$$

By the monotonicity of $s(\cdot)$, one may differentiate $k$ times to conclude that

$$
s(u) \sim A u
$$

which implies (2.4). (For such differentiation, cf. Landau and Gaier 1986].)

Derivation of the PNT. For Re $z>1$, logarithmic differentiation of the Euler product for the zeta function, $\zeta(z)=\prod_{p} 1 /\left(1-p^{-z}\right)$ over the primes $p$, gives the formula

$$
f(z)=\sum_{1}^{\infty} \frac{\Lambda(n)}{n^{z}}=-\frac{\zeta^{\prime}(z)}{\zeta(z)},
$$

where $\Lambda(n)=\log p$ if $n=p^{\beta}(\beta \geq 1)$ and $\Lambda(n)=0$ if $n$ is not a prime power. One knows that $\zeta(z)$ can be continued analytically across every point of the line $\operatorname{Re} z=1$ except the point $z=1$, where $\zeta(z)$ has a simple pole with residue 1 . We also need the crucial fact that $\zeta(\cdot)$ is free of zeros for $\operatorname{Re} z=1$. It follows that $-\zeta^{\prime}(z) / \zeta(z)$ likewise is analytic at every point of the line $\operatorname{Re} z=1$ with the exception of a simple pole at $z=1$ with residue 1 . Thus

$$
g(z)=f(z)-\frac{1}{z-1}=-\frac{\zeta^{\prime}(z)}{\zeta(z)}-\frac{1}{z-1}
$$

has an analytic extension to the closed half-plane $\operatorname{Re} z \geq 1$. If one knows that this function $g$ indeed satisfies a growth condition (2.3), the conclusion from Theorem 2.1 , cf. (2.7), is that

$$
\psi(u) \stackrel{\text { def }}{=} \sum_{n \leq u} \Lambda(n)=\sum_{p^{\alpha} \leq n} \log p \sim u .
$$

This relation is equivalent to the PNT (1.2); cf. Section 8 for details.

For a proof of a suitable growth condition we refer to Landau [1909] or Ingham [1932]. However, Ikehara's article [1931] showed that no growth condition is necessary:

Theorem 2.2. Let $\left\{a_{n}\right\}$ and $f$ be as in Theorem 2.1, and let $g(z)=f(z)-A /(z-1)$ have a continuous extension to the closed half-plane $\operatorname{Re} z \geq 1$. Then

$$
s_{n}=\sum_{1}^{n} a_{k} \sim A n \quad \text { as } n \rightarrow \infty .
$$

The condition $a_{n} \geq 0$ may be relaxed to $a_{n} \geq-C$. [One may apply the above result to $f^{*}(z)=f(z)+C \zeta(z)$.] 


\section{LAPLACE INTEGRALS}

Instead of sums of (absolutely convergent) Dirichlet series, one may more generally consider functions $f$ which for $\operatorname{Re} z>1$ are given by an absolutely convergent Mellin-Stieltjes integral

$$
f(z)=\int_{1-}^{\infty} v^{-z} d s(v)
$$

If $s(\cdot)$ is nondecreasing or if $s(v)=\mathcal{O}(v)$, one may integrate by parts to obtain an ordinary Mellin transform; cf. the paragraph below. Alternatively, one may first substitute $s\left(e^{t}\right)=S(t)$ to obtain a Laplace-Stieltjes transform $\mathcal{L} d S$ as in (1.1).

It is convenient to insert a remark here on integration by parts in general LaplaceStieltjes integrals. Always taking $S(t)=0$ for $t<0$, we suppose that $S$ is locally of bounded variation on $[0, \infty)$ and such that the integral for $\mathcal{L} d S(z)$ is absolutely convergent for $\operatorname{Re} z=x>a \geq 0$. Under this condition

$$
\mathcal{L} d S(z)=\int_{0-}^{\infty} e^{-z t} d S(t)=z \int_{0}^{\infty} S(t) e^{-z t} d t=z \mathcal{L} S(z)
$$

for $x>a$ and the final integral will also be absolutely convergent. Indeed, writing $V(t)=\int_{0-}^{t}|d S(u)|$ and taking $0<B<\infty$ one has

$$
\int_{0-}^{B} e^{-x t}|d S(t)|=e^{-B x} V(B)+x \int_{0}^{B} V(t) e^{-x t} d t .
$$

Since the second term is $\geq 0$, the hypothesis implies that the last term has a finite limit for $B \rightarrow \infty$. Thus the second term also has a limit and it must be 0 , or else the last term would tend to $\infty$. It follows that $e^{-B x} S(B) \rightarrow 0$. The analog of (3.3) with $d S$ and $S$ instead of $|d S|$ and $V$ now gives (3.2).

We end with a continuity theorem in preparation for the Wiener-Ikehara theorem in Section 4. If $s(v)=A v$, the transform $f(z)$ in (3.1) is equal to $A /(z-1)$ for $x=\operatorname{Re} z>1$. What can one say if $s(v) \sim A v$ ?

Proposition 3.1. Suppose that $s(\cdot)$ is locally of bounded variation, $s(v)=0$ for $v<1$ and $s(v) \sim A v$ as $v \rightarrow \infty$. Then the analytic function $f(z)$ defined by (3.1) for $x=\operatorname{Re} z>1$ behaves like $A /(z-1)$ at least for so-called angular approach to the point $z=1$. There can be no pole type behavior for angular approach to any other point of the line $\operatorname{Re} z=1$.

Proof. Using integration by parts one finds that for $x=\operatorname{Re} z>1$,

$$
g(z)=f(z)-\frac{A}{z-1}=z \int_{1}^{\infty}\{s(v)-A v\} v^{-z-1} d v .
$$

The hypothesis $s(v) \sim A v$ implies that for any $\varepsilon>0$, there is a constant $B \geq 1$ such that $|s(v)-A v|<\varepsilon v$ for $v \geq B$. Then for $z$ in the angle given by $1<x<2$ and $|z-1|<C_{1}(x-1)$,

$$
\begin{aligned}
|g(z)| & \leq|z|\left\{\int_{1}^{B}|s(v)-A v| v^{-x-1} d v+\varepsilon \int_{B}^{\infty} v^{-x} d v\right\} \\
& \leq C_{2}+\frac{\varepsilon}{x-1} \leq C_{2}+\frac{C_{1} \varepsilon}{|z-1|} .
\end{aligned}
$$

It follows that $(z-1) g(z) \rightarrow 0$ and $(z-1) f(z) \rightarrow A$ as $z \rightarrow 1$ in the angle. 
We now take $z$ in an angle of the form $1<x<2$ and $\left|z-z_{0}\right|<C_{1}(x-1)$ where $z_{0}=1+i y_{0}$ with $y_{0} \neq 0$. Then $|g(z)|$ satisfies an inequality of the form (3.5) with denominator $\left|z-z_{0}\right|$ in the final term instead of $|z-1|$. In that case one finds that

$$
\left|\left(z-z_{0}\right) f(z)\right|=\left|\left(z-z_{0}\right) g(z)+A\left(z-z_{0}\right) /(z-1)\right|<C_{3} \varepsilon
$$

when $x$ is close to 1 . Conclusion: $\left(z-z_{0}\right) f(z) \rightarrow 0$ as $z \rightarrow z_{0}$ in the angle with vertex $z_{0}$.

Corollary 3.2. The prime number theorem implies that $\zeta(z)$ is free of zeros on the line $\operatorname{Re} z=1$.

Indeed, if $s(v)=\psi(v)$ in Proposition 3.1, then $f(z)=-\zeta^{\prime}(z) / \zeta(z)$ (Section 2). Now by the $\operatorname{PNT} \psi(v) \sim v$. Thus $f$ can have no pole on the line Re $z=1$ (different from the point 1 ), so that $\zeta$ can have no zero there.

\section{The Wiener-IKehara theorem}

We consider an integral form of Theorem 2.2:

Theorem 4.1. Let $s(v)$ vanish for $v<1$, be nonnegative and nondecreasing on $[1, \infty)$ and such that the Mellin-Stieltjes transform

$$
f(z)=\int_{1-}^{\infty} v^{-z} d s(v)=z \int_{1}^{\infty} s(v) v^{-z-1} d v, \quad z=x+i y
$$

exists for $\operatorname{Re} z=x>1$. Suppose that for some constant $A$, the analytic function

$$
g(z)=f(z)-\frac{A}{z-1}, \quad x>1
$$

has a continuous extension to the closed half-plane $x \geq 1$. Then

$$
s(u) / u \rightarrow A \quad \text { as } u \rightarrow \infty .
$$

For the integration by parts in (4.1), cf. Section 3. In the theorem above it is sufficient to have $s(v)+C v$ nonnegative and nondecreasing for some constant $C$.

It is convenient to deal with a corresponding (slightly more general) result for Laplace-Stieltjes transforms. For the transition we set $v=e^{t}, s\left(e^{t}\right)=S(t)$.

Theorem 4.2. Let $S(t)$ vanish for $t<0$, be nonnegative and nondecreasing for $t \geq 0$ and such that the Laplace-Stieltjes transform

$$
f(z)=\mathcal{L} d S(z)=\int_{0-}^{\infty} e^{-z t} d S(t)=z \int_{0}^{\infty} S(t) e^{-z t} d t, \quad z=x+i y
$$

exists for $\operatorname{Re} z=x>1$. Suppose that for some constant $A$, the analytic function

$$
g(z)=f(z)-\frac{A}{z-1}, \quad x>1
$$

has a boundary function $g(1+i y)$ in the following sense. For $x \backslash 1$, the function $g_{x}(i y)=g(x+i y)$ converges to $g(1+i y)$ uniformly or in $L^{1}$ on every finite interval $-\lambda<y<\lambda$. Then

$$
e^{-t} S(t) \rightarrow A \quad \text { as } t \rightarrow \infty
$$


Working with Wiener, Ikehara applied his mentor's early [1928] Tauberian theory to obtain a proof [1931]. Thus their joint effort succeeded in removing the earlier growth condition on $f$ or $g$ at $\infty$ which was imposed in Section 2. Most subsequent proofs and extensions have benefited from Wiener's version [1932] of the proof and from Bochner's simplification [1933. of it. See Landau 1932a, Ingham 1935, Wiener and Pitt [1939, as well as expositions in books by Wiener [1933, Doetsch [1937], 1950], Widder [1941], Chandrasekharan [1968], and Heins [1968].

We give two proofs for the Wiener-Ikehara theorem. The proof below is along traditional lines. The elegant newer proof in Section 5 gives an optimal result for the case where there is a good boundary function $g(1+i y)$ only on some finite interval $-\lambda<y<\lambda$. Less precise results for that case were obtained earlier by Heilbronn and Landau 1933a, [1933c; see also Landau [1932b].

Following Wiener 1932] and Bochner [1933], we base our first proof of Theorem 4.2 on a nonnegative approximate identity $\left\{K_{\lambda}\right\}, 0<\lambda \rightarrow \infty$ such that the Fourier transform $\hat{K}_{\lambda}$ has support in $[-\lambda, \lambda]$. It is convenient to use the historical example of the 'Fejér kernel':

$$
\begin{aligned}
& K_{\lambda}(t)=\lambda K(\lambda t)=\frac{1-\cos \lambda t}{\pi \lambda t^{2}}=\frac{\lambda}{2 \pi}\left(\frac{\sin \lambda t / 2}{\lambda t / 2}\right)^{2}, \\
& \hat{K}_{\lambda}(y)=\int_{\mathbb{R}} K_{\lambda}(t) e^{-i y t} d t= \begin{cases}1-|y| / \lambda & \text { for }|y| \leq \lambda, \\
0 & \text { for }|y|>\lambda .\end{cases}
\end{aligned}
$$

Proposition 4.3. Let $\sigma(t)$ vanish for $t<0$, be nonnegative for $t \geq 0$ and such that the Laplace transform

$$
F(z)=\mathcal{L} \sigma(z)=\int_{0}^{\infty} \sigma(t) e^{-z t} d t
$$

exists for $\operatorname{Re} z=x>0$. Let $\lambda>0$ be given. Suppose that for $x \searrow 0$, the function

$$
G(z)=F(z)-A / z, \quad z=x+i y
$$

converges to a boundary function $G(i y)$ in $L^{1}$ sense for $-\lambda<y<\lambda$. Then the integral

$$
\begin{aligned}
& \int_{\mathbb{R}} K_{\lambda}(u-t) \sigma(t) d t=\int_{-\infty}^{\lambda u} \sigma(u-v / \lambda) K(v) d v \\
& \text { exists and tends to } A \int_{\mathbb{R}} K(v) d v=A \quad \text { as } u \rightarrow \infty .
\end{aligned}
$$

Proof. For $x>0$ the convolution of $K_{\lambda}(t)$ and $\sigma(t) e^{-x t}$ has Fourier transform $\hat{K}_{\lambda}(y) F(x+i y)$, so that by Fourier inversion

$$
\int_{\mathbb{R}} K_{\lambda}(u-t) \sigma(t) e^{-x t} d t=\frac{1}{2 \pi} \int_{-\lambda}^{\lambda} \hat{K}_{\lambda}(y) F(x+i y) e^{i u y} d y .
$$

The special function $F_{1}(x+i y)=1 /(x+i y)$ is the Laplace transform of the function $\sigma_{1}$ which equals 1 for $t \geq 0$ and 0 for $t<0$. Hence by (4.11) for $\sigma_{1}$ and $F_{1}$,

$$
\int_{0}^{\infty} K_{\lambda}(u-t) e^{-x t} d t=\frac{1}{2 \pi} \int_{-\lambda}^{\lambda} \hat{K}_{\lambda}(y) F_{1}(x+i y) e^{i u y} d y
$$


One now subtracts $A$ times this formula from (4.11) and then replaces $F-A F_{1}$ by $G$. The result may be written as

$$
\begin{aligned}
\int_{0}^{\infty} K_{\lambda}(u-t) \sigma(t) e^{-x t} d t= & A \int_{0}^{\infty} K_{\lambda}(u-t) e^{-x t} d t \\
& +\frac{1}{2 \pi} \int_{-\lambda}^{\lambda} \hat{K}_{\lambda}(y) G(x+i y) e^{i u y} d y .
\end{aligned}
$$

By the hypotheses, the right-hand side of (4.13) has a finite limit as $x \searrow 0$; hence so does the left-hand side. Thus by the monotone convergence theorem, the nonnegative product $K_{\lambda}(u-t) \sigma(t)$ is integrable over $(0, \infty)$. Letting $x \searrow 0$, one obtains the 'basic relation' in the proofs by Wiener and Bochner,

$$
\int_{0}^{\infty} K_{\lambda}(u-t) \sigma(t) d t=A \int_{0}^{\infty} K_{\lambda}(u-t) d t+\frac{1}{2 \pi} \int_{-\lambda}^{\lambda} \hat{K}_{\lambda}(y) G(i y) e^{i u y} d y
$$

Application of the Riemann-Lebesgue lemma finally shows that the last term tends to 0 as $u \rightarrow \infty$. Substituting $u-t=v / \lambda$ and replacing $K_{\lambda}(v / \lambda)$ by $\lambda K(v)$, one concludes that

$$
\lim _{u \rightarrow \infty} \int_{-\infty}^{\lambda u} \sigma(u-v / \lambda) K(v) d v=A \lim _{u \rightarrow \infty} \int_{-\infty}^{\lambda u} K(v) d v=A .
$$

Proof of Theorem 4.2. We set $e^{-t} S(t)=\sigma(t)$. Then for $\operatorname{Re} z=x>0$,

$$
\begin{aligned}
& F(z) \stackrel{\text { def }}{=} \mathcal{L} \sigma(z)=\int_{0}^{\infty} S(t) e^{-(z+1) t} d t=\frac{f(z+1)}{z+1}, \\
& G(z) \stackrel{\text { def }}{=} F(z)-\frac{A}{z}=\frac{f(z+1)}{z+1}-\frac{A}{z}=\frac{g(z+1)-A}{z+1} ;
\end{aligned}
$$

cf. (4.4), (4.5). By the hypotheses of the theorem, $\sigma, F$ and $G$ will satisfy the conditions of Proposition 4.3; hence we have (4.10). Because $\left\{K_{\lambda}\right\}$ is an approximate identity, the left-hand side of (4.10) must be close to $\sigma(u)$ if $\sigma$ is well-behaved and $\lambda$ is large. But is our $\sigma$ nice enough?

At this point Wiener 1932 continued with 'Wiener theory', but Bochner's method (we give Landau's version) was more direct. By the monotonicity of $S$, one has $\sigma\left(v^{\prime}\right) \geq \sigma(v) e^{v-v^{\prime}}$ if $v^{\prime} \geq v$. For any $a>0$, (4.10) thus shows that

$$
\begin{aligned}
A & =\lim _{u \rightarrow \infty} \int_{-\infty}^{\lambda u} \sigma(u-v / \lambda) K(v) d v \geq \limsup _{u \rightarrow \infty} \int_{-a}^{a} \sigma(u-v / \lambda) K(v) d v \\
& \geq \limsup _{u \rightarrow \infty} \sigma(u-a / \lambda) e^{-2 a / \lambda} \int_{-a}^{a} K(v) d v .
\end{aligned}
$$

As a result,

$$
\limsup _{u \rightarrow \infty} \sigma(u) \leq \frac{e^{2 a / \lambda}}{\int_{-a}^{a} K(v) d v} A
$$

Taking $a=\sqrt{\lambda}$ one obtains an upper bound for the limsup of the form $C(\lambda) A$, where $C(\lambda)$ is close to 1 for large $\lambda$. Under the hypotheses of Theorem 4.2 we may indeed let $\lambda$ go to $\infty$ to conclude that

$$
\limsup _{u \rightarrow \infty} \sigma(u) \leq A .
$$


Letting $\lambda$ go to $\infty$ or not, we know now that $\sigma$ is bounded by some constant $M$. Taking $b>0$ and observing that $K(v) \leq 2 /\left(\pi v^{2}\right)<1 / v^{2}$, one obtains an estimate from below:

$$
\begin{aligned}
& \liminf _{u \rightarrow \infty} \sigma(u+b / \lambda) e^{2 b / \lambda} \int_{-b}^{b} K(v) d v \geq \liminf _{u \rightarrow \infty} \int_{-b}^{b} \sigma(u-v / \lambda) K(v) d v \\
& \geq \lim _{u \rightarrow \infty} \int_{\mathbb{R}} \cdots-\limsup _{u \rightarrow \infty} \int_{-\infty}^{-b} \cdots-\limsup _{u \rightarrow \infty} \int_{b}^{\infty} \cdots \\
& \geq A-2 M \int_{b}^{\infty}\left(1 / v^{2}\right) d v=A-2 M / b .
\end{aligned}
$$

This gives a lower bound for $\liminf _{u \rightarrow \infty} \sigma(u)$ which for large $\lambda$ and related large $b$ is as close to $A$ as one wishes. Conclusion:

$$
\liminf _{u \rightarrow \infty} \sigma(u) \geq A \text {. }
$$

For fixed $\lambda$ more precise analysis would give a lower bound for the lim inf of the form $c(\lambda) A$, where $c(\lambda)$ is close to 1 if $\lambda$ is large; cf. Landau 1932b. A sharp 'finite form' of the theorem is given in the next section.

Remarks 4.4. Many authors have obtained extensions of the Wiener-Ikehara theorem involving a variety of boundary behavior, among them Raikov [1938, Ingham [1941], Agmon [1953], Delange [1954], [1955], Subhankulov [1973], [1976, chapter 5], Graham and Vaaler [1981], Aramaki [1996], and Č́́žek 1999]. Among other things, Subhankulov allowed finite sums in (4.2) of the form $\sum_{m>1} A_{m} z /(z-1)^{m}$ instead of $A /(z-1)$; see also Č́žzek. Tenenbaum [1995] derived a form of the Wiener-Ikehara theorem with remainder.

In the special case of Dirichlet series (2.1) with multiplicative coefficients $a_{n}=$ $\phi(n)$ of absolute value $\leq 1$, Halász [1968] could use the behavior of $f(z)=f(x+i y)$ near the line $x=1$ to obtain very refined results on the partial sums $s_{n}=\sum_{k \leq n} a_{k}$. He could thus extend earlier number theoretic work on the mean values of arithmetic functions due to Delange and Wirsing; cf. Elliott [1979].

\section{NEWER APPROACH TO WIENER-IKEHARA}

The treatment below is due to Graham and Vaaler [1981. They were able to obtain a precise 'finite form' of the Wiener-Ikehara theorem with the aid of 'extremal majorants'. The majorants are optimal among (the restrictions to $\mathbb{R}$ of) entire functions of prescribed exponential type. For some simple step functions such majorants had been considered earlier by Beurling (unpublished) and Selberg; see his Collected papers, vol. 2 [1991, pp. 213-218, 225-226] and cf. Montgomery 1978, lemma 5] and [1994.

Extending this work, Graham and Vaaler obtained extremal majorants for a larger class of functions; cf. also Vaaler [1985, Holt and Vaaler [1996]. For the present application, the relevant function is

$$
E(t)= \begin{cases}e^{-t} & \text { for } t \geq 0 \\ 0 & \text { for } t<0\end{cases}
$$

Taking $\lambda>0$ we consider majorants and minorants for $E$ of type $\lambda$. By this we mean integrable majorants $M_{\lambda}=M_{\lambda, E}$ and minorants $m_{\lambda}=m_{\lambda, E}$ on $\mathbb{R}$ which 


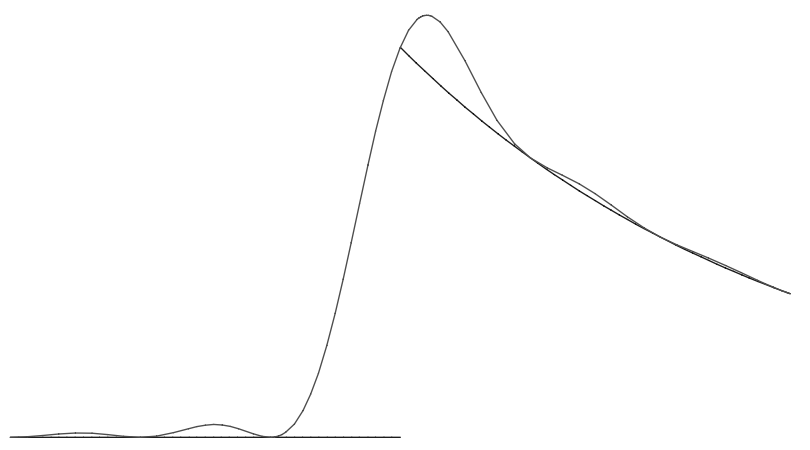

Figure 1. $E$ and $M_{\lambda}^{*}$

are the restrictions of entire functions of exponential type $\leq \lambda$. Equivalently, the Fourier transforms $\hat{M}_{\lambda}$ and $\hat{m}_{\lambda}$ have support in $[-\lambda, \lambda]$; cf. Paley and Wiener [1934].

Theorem 5.1. Let $S(t)$ vanish for $t<0$, be nonnegative and nondecreasing for $t \geq 0$ and such that the Laplace-Stieltjes transform

$$
f(z)=\mathcal{L} d S(z)=\int_{0-}^{\infty} e^{-z t} d S(t), \quad z=x+i y
$$

exists for $\operatorname{Re} z=x>1$. Suppose that there is a constant $A$ (necessarily $\geq 0$ ) such that the analytic function

$$
g(z)=f(z)-\frac{A}{z-1}, \quad z=x+i y, \quad x>1
$$

converges to a boundary function $g(1+i y)$ in $L^{1}(-\lambda<y<\lambda)$ as $x \backslash 1$. Then for any majorant $M_{\lambda}=M_{\lambda, E}$ and minorant $m_{\lambda}=m_{\lambda, E}$,

$$
A \int_{\mathbb{R}} m_{\lambda}(t) d t \leq \liminf _{t \rightarrow \infty} e^{-t} S(t) \leq \limsup _{t \rightarrow \infty} e^{-t} S(t) \leq A \int_{\mathbb{R}} M_{\lambda}(t) d t .
$$

Proposition 5.2. Among the majorants $M_{\lambda}=M_{\lambda, E}$ of $E$ is the function

$$
M_{\lambda}^{*}(t)=\left(\frac{\sin \lambda t / 2}{\lambda / 2}\right)^{2}\left\{\sum_{n=0}^{\infty} \frac{e^{-n \omega}}{(t-n \omega)^{2}}-\sum_{n=1}^{\infty} e^{-n \omega}\left(\frac{1}{t-n \omega}-\frac{1}{t}\right)\right\},
$$

where $\omega=2 \pi / \lambda$; see Figure 1. One has

$$
\int_{\mathbb{R}} M_{\lambda}^{*}(t) d t=\frac{\omega}{1-e^{-\omega}}=\frac{2 \pi / \lambda}{1-e^{-2 \pi / \lambda}} .
$$

Among the minorants $m_{\lambda}=m_{\lambda, E}$ is the function

$$
m_{\lambda}^{*}(t)=M_{\lambda}^{*}(t)-\left(\frac{\sin \lambda t / 2}{\lambda t / 2}\right)^{2},
$$

for which

$$
\int_{\mathbb{R}} m_{\lambda}^{*}(t) d t=\frac{\omega}{e^{\omega}-1}=\frac{2 \pi / \lambda}{e^{2 \pi / \lambda}-1} .
$$

Remarks 5.3. The proof of the proposition will show that $M_{\lambda}^{*}$ is an interpolant of $E$ on the sequence $\{n \omega\}$ in the strong sense that

$$
M_{\lambda}^{*}(n \omega)=E(n \omega), \quad \forall n \quad \text { and } \quad\left(M_{\lambda}^{*}\right)^{\prime}(n \omega)=E^{\prime}(n \omega), \quad \forall n \neq 0
$$


(Figure 1). Similarly $m_{\lambda}^{*}$ is a strong interpolant of the function $\tilde{E}$ which is equal to $E$ for $t \neq 0$, but equal to 0 for $t=0$.

For a class of upper semicontinuous $L^{1}$ functions $F$, including $F(t)=E(\omega t)$, Graham and Vaaler had obtained 'extremal majorants' of type $2 \pi$. The general form is

$$
M_{F}^{*}(t)=\left(\frac{\sin \pi t}{\pi}\right)^{2}\left\{\sum_{n=-\infty}^{\infty} \frac{F(n)}{(t-n)^{2}}+\sum_{n \neq 0} F^{\prime}(n)\left(\frac{1}{t-n}-\frac{1}{t}\right)\right\} .
$$

One may derive from their work that $M_{\lambda}^{*}$ in (5.5) is the unique extremal majorant for $E$ of type $\lambda$; it minimizes $\int_{\mathbb{R}}\left(M_{\lambda}-E\right)$ or, equivalently, minimizes $\hat{M}_{\lambda}(0)=$ $\int_{\mathbb{R}} M_{\lambda}$. Similarly $m_{\lambda}^{*}$ is the unique extremal minorant for $E$ of type $\lambda$; it minimizes $\int_{\mathbb{R}}\left(E-m_{\lambda}\right)$ or maximizes $\hat{m}_{\lambda}(0)$.

Theorem 5.4. Let $S$ and $f=\mathcal{L} d S$ be as in Theorem 5.1 and let $g(z)=f(z)-$ $A /(z-1)$. Let $\mu>0$ be the supremum of the numbers $\lambda$ such that $g(x+i y)$ converges to a boundary function $g(1+i y)$ in $L^{1}(-\lambda<y<\lambda)$ as $x \searrow 1$. Then

$$
\frac{2 \pi / \mu}{e^{2 \pi / \mu}-1} A \leq \liminf _{t \rightarrow \infty} e^{-t} S(t) \leq \limsup _{t \rightarrow \infty} e^{-t} S(t) \leq \frac{2 \pi / \mu}{1-e^{-2 \pi / \mu}} A
$$

where the bounds should be read as $A$ if $\mu=\infty$. The bounds in (5.11) are sharp.

Proof of Theorem 5.1. Aiming for an upper bound for $e^{-t} S(t)=e^{-t} \int_{0-}^{t} d S(u)$, we initially insert a factor $e^{-(x-1) u}$ with $x>1$ into the integral to facilitate the analysis. By the definitions of $E$ and a majorant $M_{\lambda}$ of $E$,

$$
\begin{aligned}
& e^{-t} \int_{0-}^{t} e^{-(x-1) u} d S(u)=\int_{0-}^{t} e^{-(t-u)} e^{-x u} d S(u) \\
& =\int_{0-}^{\infty} E(t-u) e^{-x u} d S(u) \leq \int_{0-}^{\infty} M_{\lambda}(t-u) e^{-x u} d S(u) .
\end{aligned}
$$

The Fourier transform $\hat{M}_{\lambda}$ is continuous and has support in $[-\lambda, \lambda]$. Since $M_{\lambda}$ is smooth it is pointwise equal to the inverse Fourier transform of $\hat{M}_{\lambda}$ :

$$
M_{\lambda}(t-u)=\frac{1}{2 \pi} \int_{-\lambda}^{\lambda} \hat{M}_{\lambda}(y) e^{i(t-u) y} d y .
$$

Substitution in (5.12), inversion of the order of integration and the definition $\mathcal{L} d S=$ $f$ now give

$$
\begin{aligned}
\int_{0-}^{\infty} M_{\lambda}(t-u) e^{-x u} d S(u) & =\frac{1}{2 \pi} \int_{-\lambda}^{\lambda} \hat{M}_{\lambda}(y) e^{i t y} d y \int_{0-}^{\infty} e^{-(x+i y) u} d S(u) \\
& =\frac{1}{2 \pi} \int_{-\lambda}^{\lambda} \hat{M}_{\lambda}(y) f(x+i y) e^{i t y} d y
\end{aligned}
$$

Observe that the 'singular part' of $f(x+i y)$ for $x \searrow 1$ and $-\lambda<y<\lambda$ is $A /(x+i y-1)$, which is equal to $\int_{0}^{\infty} e^{-(x+i y) u} A e^{u} d u$. In the special case where $S(u)=A e^{u}$ for $u \geq 0$, identity (5.13) shows that

$$
\frac{1}{2 \pi} \int_{-\lambda}^{\lambda} \hat{M}_{\lambda}(y) \frac{A}{x+i y-1} e^{i t y} d y=\int_{0}^{\infty} M_{\lambda}(t-u) e^{-x u} A e^{u} d u .
$$


Combining (5.12) - (5.14) with the relation $f=g+$ singular part, one thus obtains the inequality

$$
\begin{aligned}
& e^{-t} \int_{0-}^{t} e^{-(x-1) u} d S(u) \\
& \leq \frac{1}{2 \pi} \int_{-\lambda}^{\lambda} \hat{M}_{\lambda}(y) g(x+i y) e^{i t y} d y+A \int_{0}^{\infty} M_{\lambda}(t-u) e^{-(x-1) u} d u
\end{aligned}
$$

At this point one can take the limit as $x \searrow 1$ because $g(x+i y)$ has 'good' boundary behavior for $-\lambda<y<\lambda$ and $M_{\lambda}$ is in $L^{1}$. The result is

$$
e^{-t} S(t) \leq \frac{1}{2 \pi} \int_{-\lambda}^{\lambda} \hat{M}_{\lambda}(y) g(1+i y) e^{i t y} d y+A \int_{0}^{\infty} M_{\lambda}(t-u) d u
$$

One may finally let $t$ go to $\infty$. The first integral in (5.15) involves the integrable function $\hat{M}_{\lambda}(y) g(1+i y)$ and the factor $e^{i t y}$; hence by the Riemann-Lebesgue lemma, it has limit 0 . The final term is equal to

$$
A \int_{-\infty}^{t} M_{\lambda}(v) d v, \quad \text { with limit } A \int_{\mathbb{R}} M_{\lambda}(v) d v .
$$

Thus for $t \rightarrow \infty$, (5.15) gives the desired upper bound for the limsup in (5.4):

$$
\limsup _{t \rightarrow \infty} e^{-t} S(t) \leq A \int_{\mathbb{R}} M_{\lambda}(v) d v
$$

The proof for the lower bound of the liminf is similar.

We will deal with Proposition 5.2 later.

Derivation of Theorem 5.4. By Theorem 5.1 applied to the majorant $M_{\lambda}^{*}$ in (5.5) and by (5.6),

$$
\limsup _{t \rightarrow \infty} e^{-t} S(t) \leq A \int_{\mathbb{R}} M_{\lambda}^{*}(t) d t=\frac{2 \pi / \lambda}{1-e^{-2 \pi / \lambda}} A .
$$

By the hypothesis this holds for every $\lambda<\mu$ and thus one obtains the (smaller) upper bound in (5.11). The proof for the lower bound of the lim inf is similar.

To show that the bounds are sharp, Graham and Vaaler used the measure $d S$ defined by point masses $A(2 \pi / \mu) e^{2 \pi k / \mu}$ at the points $2 \pi k / \mu$ for $k=1,2, \ldots$ In this case $f=\mathcal{L} d S$ has simple poles with residue $A$ at the points $z=1+n \mu i, n \in \mathbb{Z}$, so that the hypotheses of Theorem 5.4 are satisfied. At the same time, the first and the last inequality in (5.11) will be equalities.

Proof of Proposition 5.2. It will suffice to consider majorants.

(i) The change of scale $t \Rightarrow \omega t$ replaces the problem to majorize $E(t)$ by functions $M_{\lambda}(t)$ of type $\lambda$ by the equivalent problem to majorize $F(t)=E(\omega t)$ by functions of type $\omega \lambda=2 \pi$. We thus set out to show that

$$
\begin{aligned}
& M^{*}(t)=\left(\frac{\sin \pi t}{\pi}\right)^{2} Q(t), \quad \text { with } \\
& Q(t)=\sum_{n=0}^{\infty} \frac{e^{-n \omega}}{(t-n)^{2}}+\sum_{n=1}^{\infty}(-\omega) e^{-n \omega}\left(\frac{1}{t-n}-\frac{1}{t}\right),
\end{aligned}
$$

is a majorant for the function $E(\omega t)$ which is equal to $e^{-\omega t}$ for $t \geq 0$ and equal to 0 for $t<0$. 
(ii) To this end, cf. Graham and Vaaler, we introduce the auxiliary function

$$
R(v)=\frac{v}{1-e^{-v}}, \quad \text { for which } 0<R^{\prime}(v)<1, \quad \forall v \in \mathbb{R} .
$$

Straightforward calculation gives the following formulas for $t<0$ :

$$
\begin{aligned}
& \int_{0}^{\infty} R(v+\omega) e^{t v} d v=e^{-\omega t} \int_{\omega}^{\infty} R(u) e^{t u} d u=\sum_{n=0}^{\infty} e^{-n \omega}\left(\frac{1}{(t-n)^{2}}-\frac{\omega}{t-n}\right), \\
& \int_{0}^{\infty} R(\omega) e^{t v} d v=-\frac{R(\omega)}{t}=-\sum_{n=0}^{\infty} e^{-n \omega} \frac{\omega}{t}, \\
& \int_{0}^{\infty}\{R(v+\omega)-R(\omega)\} e^{t v} d v=Q(t)
\end{aligned}
$$

cf. (5.17). By (5.18) $0<R(v+\omega)-R(\omega)<v$ for $v>0$; hence

$$
0<Q(t)<\int_{0}^{\infty} v e^{t v} d v=\frac{1}{t^{2}}, \quad 0 \leq M^{*}(t) \leq\left(\frac{\sin \pi t}{\pi t}\right)^{2} \quad \text { for } t<0 .
$$

Similarly for $t$ positive (but different from an integer),

$$
\begin{aligned}
& \int_{-\infty}^{0} R(v+\omega) e^{t v} d v=e^{-\omega t}\left(\int_{-\infty}^{0}+\int_{0}^{\omega}\right) R(u) e^{t u} d u \\
& =e^{-\omega t} \sum_{n=-\infty}^{\infty} \frac{1}{(t-n)^{2}}-\sum_{n=0}^{\infty} e^{-n \omega}\left(\frac{1}{(t-n)^{2}}-\frac{\omega}{t-n}\right), \\
& \int_{-\infty}^{0} R(\omega) e^{t v} d v=\frac{R(\omega)}{t}=\sum_{n=0}^{\infty} e^{-n \omega} \frac{\omega}{t}
\end{aligned}
$$

Combination of these formulas with the identity

$$
\sum_{n=-\infty}^{\infty} \frac{1}{(t-n)^{2}}=\left(\frac{\pi}{\sin \pi t}\right)^{2}
$$

shows that for nonintegral $t>0$

$$
\int_{-\infty}^{0}\{R(\omega)-R(v+\omega)\} e^{t v} d t=Q(t)-e^{-\omega t}\left(\frac{\pi}{\sin \pi t}\right)^{2} .
$$

Using the inequality $0<R(\omega)-R(v+\omega)<|v|$ for $v<0$, cf. (5.18), and multiplying through by $\{(\sin \pi t) / \pi\}^{2}$, one concludes that

$$
0 \leq M^{*}(t)-e^{-\omega t} \leq\left(\frac{\sin \pi t}{\pi t}\right)^{2} \text { for nonintegral } t>0 .
$$

By continuity this holds also for positive integers $t$.

(iii) Formulas (5.19), (5.20) show that $M^{*}(t)$ majorizes $E(\omega t)$ for all $t \neq 0$; at the origin both functions are equal to 1 . The same formulas show that $M^{*}(t)$ is a strong interpolant of $E(\omega t)$ on the integers in the sense of (5.9) (where one now has to substitute $\lambda=2 \pi, \omega=1)$.

(iv) Replacing $t$ by $t / \omega$ one concludes that $M_{\lambda}^{*}(t)=M^{*}(t / \omega)$ in formula (5.5) is a majorant for $E(t)$. It is easy to verify that $M_{\lambda}^{*}$ is in $L^{1}$. It is the restriction of an entire function of exponential type $\leq \lambda$ and hence its Fourier transform will have support in $[-\lambda, \lambda]$. The latter may be confirmed by direct computation of the 
transform. The value of $\int_{\mathbb{R}} M_{\lambda}^{*}(t) d t$ in (5.6) may be verified with the aid of the formulas

$$
\int_{\mathbb{R}}\left(\frac{\sin \lambda t / 2}{\lambda / 2}\right)^{2} \frac{d t}{(t-n \omega)^{2}}=\frac{2}{\lambda} \int_{\mathbb{R}} \frac{\sin ^{2} v}{v^{2}} d v=\frac{2 \pi}{\lambda}, \quad \int_{\mathbb{R}}\left(\frac{\sin \lambda t / 2}{\lambda / 2}\right)^{2} \frac{d t}{t-n \omega}=0 .
$$

\section{NeWman's Way to the PNT. Work of Ingham}

Newman [1980] (cf. [1998]) used contour integration to obtain a suitable auxiliary theorem for Dirichlet series:

Theorem 6.1. Let $f$ be given for $\operatorname{Re} z>1$ by a Dirichlet series $\sum_{1}^{\infty} a_{n} / n^{z}$ with $\left|a_{n}\right| \leq C$ for all $n$. Suppose that $f(z)$ has an analytic extension to a neighborhood of every point of the line $\operatorname{Re} z=1$. Then

$$
\text { the series } \sum_{1}^{\infty} \frac{a_{n}}{n} \text { is convergent. }
$$

If not $f(z)$ itself, but $g(z)=f(z)-A /(z-1)$ has an analytic extension to the closed half-plane $\operatorname{Re} z \geq 1$, or, equivalently, if

$$
h(z)=f(z)-A \zeta(z)=\sum_{1}^{\infty} \frac{a_{n}-A}{n^{z}}
$$

has such an extension, the conclusion is that

$$
\text { the series } \sum_{1}^{\infty} \frac{a_{n}-A}{n} \text { is convergent; }
$$

cf. the weaker conclusion in Theorem 2.2 (under a weaker hypothesis). Newman described two ways in which the PNT can be deduced from Theorem 6.1. The simplest was to consider the reciprocal of the zeta function. As we know, $\zeta(z)$ is analytic and different from 0 throughout the closed half-plane $\operatorname{Re} z \geq 1$, except that it has a simple pole at the point $z=1$; cf. Section 2. Thus $f(z)=1 / \zeta(z)$ is analytic for $\operatorname{Re} z \geq 1$. It can be expanded in a Dirichlet series as follows:

$$
f(z)=\frac{1}{\zeta(z)}=\prod_{p \text { prime }}\left(1-\frac{1}{p^{z}}\right)=\sum_{1}^{\infty} \frac{\mu(n)}{n^{z}} .
$$

The expansion coefficients $\mu(n)$ define the so-called Möbius function. Since $|\mu(n)| \leq$ 1 for all $n$, Theorem 6.1 shows that the series $\sum \mu(n) / n$ is convergent. From this fact the PNT may be obtained by appropriate manipulation, as was first shown by Landau [1912].

A more direct proof of the PNT may be obtained from an analog of Theorem 6.1 for Laplace integrals (Korevaar [1982]; cf. Zagier [1997, Lang [1999]). Set

$$
s(v)=\sum_{n \leq v} a_{n}, \quad v=e^{-t}, \quad e^{-t} s\left(e^{t}\right)=\alpha(t),
$$

so that boundedness of the sequence $\left\{a_{n}\right\}$ implies boundedness of the function $\alpha$. Furthermore, formal integration by parts suggests that convergence of the series 
$\sum_{1}^{\infty} a_{n} / n$ corresponds to convergence of the improper integral $\int_{0}^{\infty} \alpha(t) d t$ :

$$
\sum_{1}^{\infty} \frac{a_{n}}{n}=\int_{1-}^{\infty} \frac{d s(v)}{v}=\int_{1}^{\infty} \frac{s(v)}{v^{2}} d v=\int_{0}^{\infty} \alpha(t) d t
$$

Finally, the original Dirichlet series will correspond to a Laplace integral involving $\alpha$; see Sections 7, 8 for precise results. Going back to Dirichlet series, the condition on $\left\{a_{n}\right\}$ in Theorem 6.1 can now be relaxed to $a_{n} \geq-C$ provided $s(v)=\mathcal{O}(v)$; see Theorem 8.1. As a special case we obtain the convergence (and the sum) of the series $\sum\{\Lambda(n)-1\} / n$; the PNT is an easy consequence. Related Tauberian results for Dirichlet series have been given by Delange [1997.

With more work, the condition $\left|a_{n}\right| \leq C$ in Theorem 6.1 may actually be replaced by the single condition $a_{n} \geq-C$, provided the Dirichlet series is known to converge for $\operatorname{Re} z>1$; see Theorem 9.2.

Let us return for a moment to Theorem 6.1 with $f(z)=1 / \zeta(z)$. Translation in the vertical direction will show that the Dirichlet series $\sum_{1}^{\infty} \mu(n) / n^{1+i y}$ converges to $1 / \zeta(1+i y)$ for every real $y$. This is interesting because the Dirichlet series $\sum_{1}^{\infty} 1 / n^{1+i y}$ for $\zeta(1+i y)$ itself is divergent for every real $y$; cf. Ingham [1932]. The example

$$
f(z)=\zeta(z-i b)=\sum_{1}^{\infty} a_{n} n^{i b} / n^{z}, \quad \operatorname{Re} z>1
$$

with real $b \neq 0$ thus shows that in Theorem 6.1 it is not enough if $f(z)$ has an analytic extension to a region $\operatorname{Re} z \geq 1, z \neq 1+i b$. A pole at just one point of the line $\operatorname{Re} z=1$ is too much, but a slightly weaker singularity would be permissible; cf. Remarks 7.2.

In [1935] Ingham proved a number of complex Tauberian theorems for LaplaceStieltjes integrals which imply results for general Dirichlet series and ordinary Laplace transforms. Theorem 6.1 (also with the one-sided condition) is contained in Ingham's Theorem III and its specialization Theorem $3(l)$ for Dirichlet series. His results for Laplace transforms are essentially included in Theorems 7.1, 9.2 and 10.1 below. For his proofs, Ingham extended the method which Bochner 1933 and Heilbronn and Landau [1933a], [1933c, cf. Landau [1932a], 1932b], had used for the Wiener-Ikehara theorem. Besides the Fejér kernel (4.7), Ingham used the 'Jackson kernel', which is the (suitably normalized) square of the Fejér kernel. Cf. also Karamata [1936]. Our treatment is different, and starts with Newman's contour method.

We add the remark that Newman's complex method has recently led to refinement and extension of Ingham's results; see Sections 13, 14.

\section{LAPLACE TRANSFORMS OF BOUNDED FUNCTIONS}

We will use Newman's contour integration method [1980 to derive the following result; cf. Korevaar [1982], Zagier [1997]. The theorem itself is contained in Karamata [1934, theorem B] and Ingham [1935, theorem III].

Theorem 7.1. Let the function $\alpha(\cdot)$ vanish on $(-\infty, 0)$ and be bounded on $[0, \infty)$, so that the Laplace transform

$$
F(z)=\mathcal{L} \alpha(z)=\int_{0}^{\infty} \alpha(t) e^{-z t} d t, \quad z=x+i y
$$




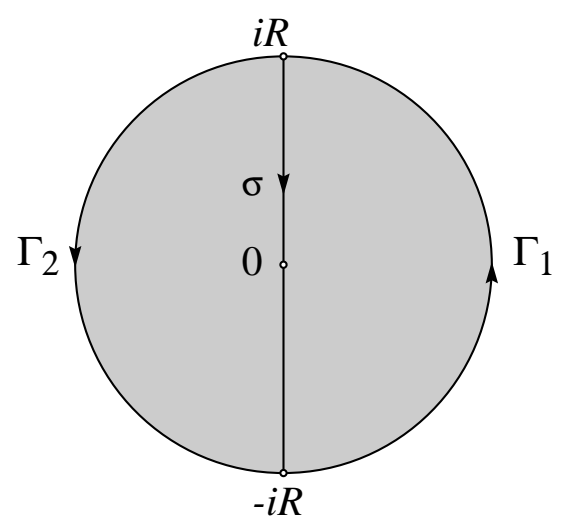

FiguRE 2. The paths of integration

is well-defined and analytic throughout the open half-plane $x=\operatorname{Re} z>0$. Suppose that $F(\cdot)$ can be continued analytically to a neighborhood of every point on the imaginary axis. Then the improper integral

$$
\int_{0}^{\infty} \alpha(t) d t \quad \text { exists and equals } F(0) .
$$

More generally, by translation in the vertical direction,

$$
\int_{0}^{\infty} \alpha(t) e^{-i y t} d t=F(i y) \quad \text { for every real } y .
$$

Proof of Theorem 7.1. Changing $\alpha$ on $[0,1]$ to $\alpha-F(0)$, the new $F=\mathcal{L} \alpha$ has $F(0)=0$. Dividing $\alpha$ by a constant, one may also assume that $\sup |\alpha(t)| \leq 1$. For $0<B<\infty$ we define

$$
F_{B}(z)=\int_{0}^{B} \alpha(t) e^{-z t} d t
$$

It must now be shown that

$$
F_{B}(0)=\int_{0}^{B} \alpha(t) d t \rightarrow F(0)=0 \quad \text { as } B \rightarrow \infty .
$$

(i) One begins with some simple estimates. For $x=\operatorname{Re} z>0$,

$$
\left|F_{B}(z)-F(z)\right|=\left|\int_{B}^{\infty} \alpha(t) e^{-z t} d t\right| \leq \int_{B}^{\infty} e^{-x t} d t=\frac{1}{x} e^{-B x} .
$$

Similarly for $x=\operatorname{Re} z<0$,

$$
\left|F_{B}(z)\right|=\left|\int_{0}^{B} \alpha(t) e^{-z t} d t\right| \leq \int_{0}^{B} e^{-x t} d t<\frac{1}{|x|} e^{-B x} .
$$

(ii) For given $R>0$, let $\Gamma$ be the positively oriented circle $|z|=R$. We let $\Gamma_{1}$ be the part of $\Gamma$ in the half-plane $x=\operatorname{Re} z>0, \Gamma_{2}$ the part in the half-plane $x<0$. Finally, let $\sigma$ be the oriented segment of the imaginary axis from $+i R$ to $-i R$ (Figure 2). Observe that for $z \in \Gamma$, one has

$$
\frac{1}{z}+\frac{z}{R^{2}}=\frac{2 x}{R^{2}}
$$


Since $F(0)=0$, the quotient $F(z) / z$ is analytic for $x \geq 0$. Observe also that $F_{B}$ is analytic everywhere. Formulas $(7.5)-(7.7)$ motivate the following ingenious application of Cauchy's theorem and Cauchy's formula due to Newman:

$$
\begin{aligned}
& 0=\frac{1}{2 \pi i} \int_{\Gamma_{1}+\sigma} \frac{F(z)}{z} d z=\frac{1}{2 \pi i} \int_{\Gamma_{1}+\sigma} F(z) e^{B z}\left(\frac{1}{z}+\frac{z}{R^{2}}\right) d z, \\
& F_{B}(0)=\frac{1}{2 \pi i} \int_{\Gamma} \frac{F_{B}(z)}{z} d z=\frac{1}{2 \pi i} \int_{\Gamma} F_{B}(z) e^{B z}\left(\frac{1}{z}+\frac{z}{R^{2}}\right) d z .
\end{aligned}
$$

Subtracting (7.8) from (7.9) and rearranging the result, one obtains the formula

$$
\begin{aligned}
F_{B}(0) & =\frac{1}{2 \pi i} \int_{\Gamma_{1}}\left\{F_{B}(z)-F(z)\right\} e^{B z}\left(\frac{1}{z}+\frac{z}{R^{2}}\right) d z \\
& +\frac{1}{2 \pi i} \int_{\Gamma_{2}} F_{B}(z) e^{B z}\left(\frac{1}{z}+\frac{z}{R^{2}}\right) d z-\frac{1}{2 \pi i} \int_{\sigma} F(z) e^{B z}\left(\frac{1}{z}+\frac{z}{R^{2}}\right) d z \\
& =T_{1}+T_{2}+T_{3},
\end{aligned}
$$

say.

(iii) By (7.5) and (7.7) for $z \in \Gamma_{1}$, the integrand $F^{*}(z)$ in $T_{1}$ can be estimated as follows:

$$
\left|F^{*}(z)\right|=\left|\left\{F_{B}(z)-F(z)\right\} e^{B z}\left(\frac{1}{z}+\frac{z}{R^{2}}\right)\right| \leq \frac{1}{x} e^{-B x} e^{B x} \frac{2 x}{R^{2}}=\frac{2}{R^{2}} .
$$

Thus

$$
\left|T_{1}\right| \leq \frac{1}{2 \pi} \int_{\Gamma_{1}}\left|F^{*}(z)\right||d z| \leq \frac{1}{2 \pi} \frac{2}{R^{2}} \pi R=\frac{1}{R} .
$$

In the same way (7.6) and (7.7) for $z \in \Gamma_{2}$ imply the estimate

$$
\left|T_{2}\right|=\left|\frac{1}{2 \pi i} \int_{\Gamma_{2}} F_{B}(z) e^{B z}\left(\frac{1}{z}+\frac{z}{R^{2}}\right) d z\right|<\frac{1}{R} .
$$

In order to deal with

$$
T_{3}=\frac{1}{2 \pi} \int_{-R}^{R} F(i y)\left(\frac{1}{i y}+\frac{i y}{R^{2}}\right) e^{i B y} d y
$$

one may apply integration by parts: $e^{i B y} d y=d e^{i B y} / i B$, etc., or one may use the Riemann-Lebesgue lemma. Either method will show that for fixed $R$,

$$
T_{3}=T_{3}(R, B) \rightarrow 0 \quad \text { as } B \rightarrow \infty .
$$

(iv) Conclusion. For given $\varepsilon>0$ one may choose $R=1 / \varepsilon$. One next determines $B_{0}$ so large that $\left|T_{3}\right|$ is bounded by $\varepsilon$ for all $B \geq B_{0}$. Then by (7.11) - (7.14),

$$
\left|F_{B}(0)\right|<3 \varepsilon \text { for } B \geq B_{0} \text {. }
$$

In other words, $F_{B}(0) \rightarrow 0=F(0)$ for $B \rightarrow \infty$ as had to be shown.

Remarks 7.2. The proof shows that one does not need analyticity of $F$ on the imaginary axis. It is enough if the quotient

$$
Q(x+i y)=\frac{F(x+i y)-F(0)}{x+i y}
$$

can be extended continuously to the closed half-plane $x \geq 0$, or if the quotient tends to a limit function $Q(i y)$ as $x \searrow 0$ in $L^{1}(-R<y<R)$ for every $R>0$. (It is 
actually enough if $Q(x+i y)$ converges distributionally to a so-called pseudofunction $Q(i y)$ on every interval $-R<y<R$; see Theorem 14.6.)

If one has $L^{1}$ convergence only on some interval $(-R, R)$, the proof above shows that

$$
\limsup _{B \rightarrow \infty}\left|F(0)-\int_{0}^{B} \alpha(t) d t\right| \leq \frac{2 M}{R}, \quad \text { where } M=\limsup _{t \rightarrow \infty}|\alpha(t)| .
$$

Here and in Theorem 7.1, the boundedness of $\alpha(t)$ for $t \geq 0$ may be relaxed to local integrability on $\mathbb{R}^{+}$and boundedness for $t \geq t_{0}$. For real $\alpha$, the boundedness condition can be relaxed further to one-sided boundedness, provided the Laplace transform $F(z)=\mathcal{L} \alpha(z)$ is known to exist for $\operatorname{Re} z>0$ (Section 9).

\section{Application to Dirichlet series and the PNT}

Theorem 7.1 implies the following result for classical Dirichlet series.

Theorem 8.1. Let $f(z)$ be given for $\operatorname{Re} z>1$ by an absolutely convergent Dirichlet series $\sum_{1}^{\infty} a_{n} / n^{z}$ with $a_{n} \geq-C$ and $s(v)=\sum_{n \leq v} a_{n}=\mathcal{O}(v)$. Suppose that $h(z)=f(z)-A \zeta(z)$ has an analytic extension to the closed half-plane $\operatorname{Re} z \geq 1$. Then

$$
\frac{1}{u} s(u) \rightarrow A \text { as } u \rightarrow \infty \text { and } \sum_{1}^{\infty} \frac{a_{n}-A}{n}=h(1) .
$$

The condition $s(v)=\mathcal{O}(v)$ may be dropped, but this requires a more complicated proof; cf. Theorem 9.2 below.

Proof of Theorem 8.1. Define

$$
\alpha(t)=\frac{s\left(e^{t}\right)-A\left[e^{t}\right]}{e^{t}}=e^{-t} \sum_{1 \leq n \leq e^{t}}\left(a_{n}-A\right) .
$$

Then $\alpha(t)=0$ for $t<0$ and $\alpha$ is bounded for $t \geq 0$. For Re $z>0$ the Laplace transform is equal to

$$
\begin{aligned}
F(z) & =\mathcal{L} \alpha(z)=\int_{0}^{\infty}\left\{s\left(e^{t}\right)-A\left[e^{t}\right]\right\} e^{-(z+1) t} d t \\
& =\int_{1}^{\infty}\{s(v)-A[v]\} v^{-z-2} d v=\frac{1}{z+1} \int_{1-}^{\infty} v^{-z-1} d\{s(v)-A[v]\} \\
& =\frac{1}{z+1} \sum_{1}^{\infty} \frac{a_{n}-A}{n^{z+1}}=\frac{f(z+1)-A \zeta(z+1)}{z+1}=\frac{h(z+1)}{z+1} .
\end{aligned}
$$

By the hypotheses the transform has an analytic extension to the closed half-plane $\operatorname{Re} z \geq 0$. Thus by Theorem 7.1

$$
\int_{0}^{\infty} \alpha(t) d t=\int_{0}^{\infty} \frac{s\left(e^{t}\right)-A\left[e^{t}\right]}{e^{t}} d t=\int_{1}^{\infty} \frac{s(v)-A[v]}{v^{2}} d v=F(0)=h(1) .
$$

(i) We will verify that the condition $a_{n} \geq-C$ and the convergence of the integrals in (8.4) imply $s(u) \sim A[u] \sim A u$.

Adding a suitable constant $C^{\prime}$ to the $a_{n}$ and $A$, we make $a_{n}>0, \forall n$ and $A>0$. This adjustment makes $s(u)$ nondecreasing. It increases $s(u)$ by $C^{\prime}[u]$ but does not change the integrals in (8.4). Suppose now that $\lim \sup s(u) / u>A$. Then 
there is a number $\delta>0$ such that for some sequence of $u$ 's tending to $\infty$, one has $s(u)>(A+2 \delta) u$. As a result,

$$
s(v) \geq s(u)>(A+2 \delta) u>(A+\delta) v \text { for } \quad u<v<\rho u,
$$

where $\rho=(A+2 \delta) /(A+\delta)$. But then for these $u \rightarrow \infty$,

$$
\int_{u}^{\rho u} \frac{s(v)-A[v]}{v^{2}} d v>\int_{u}^{\rho u} \frac{\delta}{v} d v=\delta \log \rho
$$

and this would contradict the convergence of the third integral in (8.4). Thus $\lim \sup s(u) / u \leq A$.

One similarly shows that one cannot have $\liminf s(u) / u<A$.

(ii) To deal with the sum in (8.1) we use a Stieltjes integral and integration by parts, together with (8.4) and the relation $s(u) \sim A u$ :

$$
\begin{aligned}
\sum_{n \leq N} \frac{a_{n}-A}{n} & =\int_{1-}^{N} \frac{1}{v} d\{s(v)-A[v]\} \\
& =\frac{s(N)-A N}{N}+\int_{1}^{N} \frac{s(v)-A[v]}{v^{2}} d v \rightarrow h(1) \quad \text { as } N \rightarrow \infty .
\end{aligned}
$$

Corollary 8.2. As usual, let $\psi(u)=\sum_{n \leq u} \Lambda(n)$ and let $\gamma$ be Euler's constant. Then

$$
\psi(u) \sim u \quad \text { as } u \rightarrow \infty \text { and } \quad \sum_{1}^{\infty} \frac{\Lambda(n)-1}{n}=-2 \gamma .
$$

It is known that the relation $\psi(u) \sim u$ is equivalent to the prime number theorem, $\pi(u) \sim u / \log u(1.2)$. Indeed, $\psi(t)$ jumps by $\log p$ at $t=p^{\beta}$. Hence by integration by parts,

$$
\sum_{p^{\beta} \leq u} \frac{1}{\beta}=\int_{2-}^{u} \frac{1}{\log t} d \psi(t)=\frac{\psi(u)}{\log u}+\int_{2}^{u} \frac{\psi(t)}{t \log ^{2} t} d t \sim \frac{u}{\log u}
$$

and

$$
\sum_{p^{\beta} \leq u} \frac{1}{\beta}-\pi(u)=\frac{1}{2} \pi\left(u^{\frac{1}{2}}\right)+\frac{1}{3} \pi\left(u^{\frac{1}{3}}\right)+\cdots \ll(\log u) \pi\left(u^{\frac{1}{2}}\right)=o\left(\frac{u}{\log u}\right) .
$$

Proof of the corollary. We apply Theorem 8.1 with $a_{n}=\Lambda(n)$ so that $f(z)=$ $-\zeta^{\prime}(z) / \zeta(z)$ and $s(u)=\psi(u)$ (Section 2). The boundedness of $s(u) / u$ follows from Chebyshev's classical estimate $\psi(u)=\mathcal{O}(u)$; cf. Landau [1909]. Since $f(z)$ and $\zeta(z)$ both have a first order pole at $z=1$ with residue 1 , the difference

$$
h(z)=f(z)-\zeta(z)=-\frac{\zeta^{\prime}(z)}{\zeta(z)}-\zeta(z)
$$

has an analytic extension to the closed half-plane Re $z \geq 1$; cf. Section 2 . Thus we may apply Theorem 8.1 with $A=1$. Formula (8.1) now implies the first relation (8.5) and the convergence of the series. The sum $h(1)$ of the series may be obtained from the expansion of the zeta function around the point $z=1$ :

$$
\zeta(z)=\frac{1}{z-1}+\gamma+c_{1}(z-1)+\ldots, \quad \zeta^{\prime}(z)=-1 /(z-1)^{2}+c_{1}+\ldots ;
$$


cf. Landau [1909]. Thus

$$
h(1)=-\lim _{z \rightarrow 1}\left\{\frac{\zeta^{\prime}(z)}{\zeta(z)}+\zeta(z)\right\}=-2 \gamma .
$$

\section{LAPLACE TRANSFORMS OF FUNCTIONS BOUNDED FROM BELOW}

We begin with a simple addition to Proposition 4.3.

Lemma 9.1. Let $\sigma \geq 0, F=\mathcal{L} \sigma, G(z)=F(z)-A / z$ and $\lambda>0$ be as in Proposition 4.3 , so that in particular $G(x+i y) \rightarrow G(i y)$ in $L^{1}(-\lambda<y<\lambda)$ as $x \searrow 0$. Then for every $h \geq 2 \pi / \lambda$,

$$
\sigma_{h}(u)=\frac{1}{h} \int_{u}^{u+h} \sigma(t) d t \leq C A+o(1) \quad \text { as } u \rightarrow \infty,
$$

with an absolute constant $C<3$.

If $\lambda$ can be taken arbitrarily large, then actually $\sigma_{h}(u) \rightarrow A$ as $u \rightarrow \infty$ for every $h>0$; cf. Wiener and Pitt [1939] or Pitt [1958, section 6.1] and Diamond [1972].

Proof of the lemma. By Proposition 4.3 where $K(v)=(1-\cos v) /\left(\pi v^{2}\right)$,

$$
\int_{-\infty}^{\lambda u} \sigma(u-v / \lambda) K(v) d v=A+o(1) \quad \text { as } u \rightarrow \infty .
$$

Now $K \geq 0$, and for $|v| \leq \pi$ one has $K(v) \geq K(\pi)>0$; hence by $(9.2)$

$$
2 \pi K(\pi) \frac{\lambda}{2 \pi} \int_{-\pi / \lambda}^{\pi / \lambda} \sigma(u+w) d w=K(\pi) \int_{-\pi}^{\pi} \sigma(u-v / \lambda) d v \leq A+o(1) .
$$

This inequality implies (9.1) with $h=2 \pi / \lambda$ and $C=1 /\{2 \pi K(\pi)\}<3$. Since the hypotheses of the lemma are a fortiori satisfied for the values $\lambda^{\prime}$ of $\lambda$ smaller than the given $\lambda$, conclusion (9.1) holds also for $h>2 \pi / \lambda$.

We can now prove an extension of Theorem 7.1 involving one-sided boundedness. A different proof may be obtained from Ingham [1935, theorem III].

Theorem 9.2. Let $\alpha(t)$ vanish for $t<0$, be bounded from below for $t \geq 0$ and such that the Laplace transform $G(z)=\mathcal{L} \alpha(z), z=x+$ iy exists for $x>0$. Suppose that $G(\cdot)$ can be continued analytically to the closed half-plane $x=\operatorname{Re} z \geq 0$. Then the improper integral $\int_{0}^{\infty} \alpha(t) d t$ exists and equals $G(0)$.

The proof and Remarks 7.2 will show that one does not need analyticity of $G$ on the line $x=0$. It is enough to know that for some constant $G(0)$ and every $\lambda>0$, the quotient $\{G(x+i y)-G(0)\} /(x+i y)$ converges in $L^{1}(-\lambda<y<\lambda)$ to a limit function $H(i y)$ as $x \searrow 0$.

Proof of Theorem 9.2. Shifting $\alpha$ one may assume that $\alpha(t)=0$ for $t<1$; such a shift does not affect the value of $G(0)$ or $\int_{0}^{\infty} \alpha$. Now choose $A>0$ such that $\alpha \geq-A$ and set $\sigma=\alpha+A$ on $\mathbb{R}^{+}, \sigma=0$ on $\mathbb{R}^{-}$. Then $\sigma, F=\mathcal{L} \sigma$ and $G(z)=$ $\mathcal{L} \alpha(z)=F(z)-A / z$ satisfy the conditions of Lemma 9.1 for every $\lambda>0$.

For $0<h \leq 1$ we consider the average

$$
\alpha_{h}(u)=\frac{1}{h} \int_{u}^{u+h} \alpha(t) d t .
$$


Then $\alpha_{h}(u)=\sigma_{h}(u)-A$ with $\sigma_{h}$ as in (9.1) when $u>0$ and $\alpha_{h}(u)=0$ for $u<0$. Also

$$
\begin{aligned}
G_{h}(z) & =\mathcal{L} \alpha_{h}(z)=\frac{1}{h} \int_{0}^{\infty} e^{-z u} d u \int_{u}^{u+h} \alpha(t) d t \\
& =\frac{1}{h} \int_{0}^{\infty} \alpha(t) d t \int_{t-h}^{t} e^{-z u} d u=\frac{e^{h z}-1}{h z} G(z) .
\end{aligned}
$$

Thus $G_{h}$ can be continued analytically to the closed half-plane $\operatorname{Re} z \geq 0$ just like $G$ and $G_{h}(0)=G(0)$. By Lemma 9.1, $\sigma_{h}$ is bounded for every fixed $h$; hence the same is true for $\alpha_{h}$. We can therefore apply Theorem 7.1 to $\alpha_{h}$ and its Laplace transform to conclude that

$$
\lim _{B \rightarrow \infty} \int_{0}^{B} \alpha_{h}(u) d u=G(0) .
$$

Taking $B \geq 1$ we next compare $\int_{0}^{B} \alpha_{h}$ with $\int_{0}^{B} \alpha$. By a short calculation

$$
\begin{aligned}
R(h, B) & =\int_{0}^{B} \alpha_{h}(u) d u-\int_{0}^{B} \alpha(t) d t=\int_{0}^{B} \sigma_{h}(u) d u-\int_{0}^{B} \sigma(t) d t \\
& =-\int_{0}^{h} \sigma(t) \frac{h-t}{h} d t+\int_{B}^{B+h} \sigma(t) \frac{B+h-t}{h} d t .
\end{aligned}
$$

Now $\sigma(t)=A$ on $(0, h)$; hence by Lemma 9.1

$$
-\frac{1}{2} A h \leq R(h, B) \leq \int_{B}^{B+h} \sigma(t) d t \leq 3 A h+o(1) \quad \text { as } B \rightarrow \infty .
$$

Thus by (9.4) and (9.5),

$$
\limsup _{B \rightarrow \infty}\left|G(0)-\int_{0}^{B} \alpha(t) d t\right| \leq|R(h, B)| \leq 3 A h .
$$

Since $h$ can be taken arbitrarily small this completes the proof of the theorem.

\section{TAuberian CONDitions other than BOUndEDNESS}

In this section we use ideas from the proof of Proposition 4.3 to obtain a general convergence theorem involving Tauberian conditions.

Theorem 10.1. Let $\sigma$ be real, equal to 0 on $\mathbb{R}^{-}$and such that the Laplace transform $F(z)=\mathcal{L} \sigma(z)$ exists for $\operatorname{Re} z=x>0$. Suppose that for $x \backslash 0, F(x+i y)$ converges to a boundary function $F(i y)$ uniformly or in $L^{1}$ on some interval $-\lambda \leq y \leq \lambda$ with $\lambda>0$. Then each of the following conditions is sufficient in order that $\sigma(u)$ tend to 0 as $u \rightarrow \infty$ :

(i) $\sigma$ is slowly decreasing on $\mathbb{R}$ : for any given $\varepsilon>0$, there are constants $\delta>0$ and $B$ such that

$$
\sigma(t) \geq \sigma(u)-\varepsilon \quad \text { whenever } u+2 \delta \geq t \geq u \geq B,
$$

and $\lambda$ may be taken arbitrarily large;

$\left(i^{\prime}\right) \sigma$ is 'very slowly decreasing' on $\mathbb{R}$ :

$$
\lim \inf \{\sigma(t)-\sigma(u)\} \geq 0 \text { for } u+1 \geq t \geq u \rightarrow \infty
$$

(while $\lambda$ is fixed);

(ii) $\sigma(t)$ is piecewise constant, its intervals of constancy have length $\geq 2 \delta-o(1)$ as $t \rightarrow \infty$ and $\lambda \delta>b$, where $b$ is the constant determined by equation (10.9) 
below. (Additional work will give the optimal constant $b=\frac{1}{2} \pi$; see Section 11 and Example 10.3.)

In the case of complex $\sigma$ one may apply the Tauberian conditions to $\sigma_{1}=\operatorname{Re} \sigma$ and $\sigma_{2}=\operatorname{Im} \sigma$ separately. The transforms $F_{j}=\mathcal{L} \sigma_{j}$ both inherit the good behavior of $F$.

Convergence results under conditions related to $(\mathrm{i})$ and $\left(\mathrm{i}^{\prime}\right)$ may be found in Karamata [1934] and Ingham [1935. In Ingham [1936] there is a result on Dirichlet series corresponding to condition (ii); cf. condition (ii) in Theorem 12.2 below. See also Pitt [1958, section 6.1].

For the proof of the theorem we first establish boundedness.

Proposition 10.2. Let $\sigma$ and $F=\mathcal{L} \sigma$ be as in the first four lines of the theorem. Then each of the following conditions implies that $\sigma$ is bounded:

(i) $\sigma$ is slowly decreasing or at least (10.1) holds for some $\varepsilon, \delta, B$ (no condition on $\lambda$ required);

(ii) $\sigma$ and $\lambda$ are as in part (ii) of the theorem.

Proof of the proposition. (i) Suppose for simplicity that inequality (10.1) can be satisfied for arbitrarily small $\varepsilon$, although this is not necessary for the proof that $\sigma$ is bounded. We verify first that the product $\sigma_{x}(t)=e^{-x t} \sigma(t)$ is bounded (and in fact, tends to 0 ) as $t \rightarrow \infty$ for every $x>0$. Indeed, suppose $\sigma_{x}(u) \geq 2 \varepsilon$ for a sequence of $u \rightarrow \infty$. Then for $u+2 \delta>t \geq u \geq B$, since also $\sigma(u) \geq 2 \varepsilon$,

$$
\sigma_{x}(t) \geq e^{-x t}\{\sigma(u)-\varepsilon\}>e^{-2 x \delta} e^{-x u} \frac{1}{2} \sigma(u)=e^{-2 x \delta} \frac{1}{2} \sigma_{x}(u) \geq e^{-2 x \delta} \varepsilon .
$$

But then $\int_{u}^{u+2 \delta} \sigma_{x}(t) d t$ would fail to tend to 0 as $u \rightarrow \infty$, contradicting the convergence of $\mathcal{L} \sigma(x)$. Similarly, one cannot have $\sigma_{x}(u) \leq-2 \varepsilon$ for a sequence of $u \rightarrow \infty$.

Changing $\sigma(\cdot)$ on a finite interval if necessary, we may assume that $\sigma$ is locally bounded. Then

$$
\beta_{x}=\sup _{t>0}\left|\sigma_{x}(t)\right|<\infty, \quad \forall x>0
$$

Let $K_{\lambda}(v)=\lambda K(\lambda v)$ again be the Fejér kernel of (4.7). By the hypothesis that the Laplace transform $F(x+i y)$ of $\sigma$ has good boundary behavior for $-\lambda \leq y \leq \lambda$, there is a constant $M$ such that

$$
\left|\int_{\mathbb{R}} K_{\lambda}(v) \sigma_{x}(u+v) d v\right|=\left|\frac{1}{2 \pi} \int_{-\lambda}^{\lambda} \hat{K}_{\lambda}(y) F(x+i y) e^{i u y} d y\right| \leq M, \quad \forall x>0
$$

cf. formula (4.11). We may assume that $\beta_{x}=\sup _{t} \sigma_{x}(t)$. If $\sigma$ is unbounded (as we may suppose for the moment) and $x$ is small, then $\beta_{x}$ is large and hence the values of $t$ for which $\sigma_{x}(t)$ is close to its supremum must be large. For given $\varepsilon^{\prime}>0$ we now choose $u-\delta \geq B$ such that $\sigma_{x}(u-\delta) \geq \beta_{x}-\varepsilon^{\prime}$ and we take $u+\delta>t \geq u-\delta$. Then by (10.1), cf. (10.3),

$$
\sigma_{x}(t) \geq e^{-x t}\{\sigma(u-\delta)-\varepsilon\} \geq e^{-2 x \delta} \sigma_{x}(u-\delta)-\varepsilon \geq e^{-2 x \delta} \beta_{x}-\varepsilon-\varepsilon^{\prime} .
$$


Thus by (10.5)

$$
\begin{aligned}
M & \geq \int_{\mathbb{R}} K_{\lambda}(v) \sigma_{x}(u+v) d v \\
& \geq \int_{-\delta}^{\delta} K_{\lambda}(v) \sigma_{x}(u+v) d v-\beta_{x} \int_{|v|>\delta} K_{\lambda}(v) d v \\
& \geq\left\{e^{-2 x \delta} \beta_{x}-\varepsilon-\varepsilon^{\prime}\right\} \int_{-\delta}^{\delta} K_{\lambda}(v) d v-\beta_{x}\left(1-\int_{-\delta}^{\delta} K_{\lambda}(v) d v\right) \\
& >\left(\left(1+e^{-2 x \delta}\right) \int_{-\delta}^{\delta} K_{\lambda}(v) d v-1\right) \beta_{x}-\varepsilon-\varepsilon^{\prime}
\end{aligned}
$$

For fixed $\delta$ we may take $x=\eta / 2 \delta$, where $\eta$ is small so that $e^{-\eta}$ is close to 1 . We may then rewrite (10.6) as

$$
M>\left(\left(1+e^{-\eta}\right) \int_{-\lambda \delta}^{\lambda \delta} K(w) d w-\int_{\mathbb{R}} K(w) d w\right) \beta_{x}-\varepsilon-\varepsilon^{\prime} .
$$

Now by (10.1) we may take $\delta$ as large as we like provided we enlarge $\varepsilon$ correspondingly. For large $\delta$ and associated $x$ the coefficient of $\beta_{x}$ in (10.7) will be positive and independent of $x$.

Conclusion. For small $x>0$, the absolute value of $\sigma_{x}(t)=e^{-x t} \sigma(t)$ has upper bound $\beta_{x}=\beta$ independent of $x$; hence $\sigma(t)$ is bounded.

(ii) For piecewise constant $\sigma$ as described, the proof of (10.4) is easy and need not be given. Supposing $\sigma$ unbounded, we continue roughly as before. Decreasing the original $\delta$ just a little (if necessary), we can change $\sigma$ on a finite interval and at isolated points in such a way that all the intervals of constancy have the form $u-\delta \leq t<u^{\prime}$ with $u^{\prime} \geq u+\delta$. Choosing such an interval $\left[u-\delta, u^{\prime}\right)$ for which $\sigma_{x}(u-\delta) \geq \beta_{x}-\varepsilon^{\prime}$, we again get (10.6) and (10.7) (now with $\varepsilon=0$ ). For small $x$ or $\eta$, the coefficient of $\beta_{x}$ in (10.7) can be made positive if and only if

$$
2 \int_{-\lambda \delta}^{\lambda \delta} K(w) d w>\int_{\mathbb{R}} K(w) d w
$$

This condition requires (for the new or the original $\delta$ ) that $\lambda \delta$ be larger than the constant $b$ for which

$$
2 \int_{-b}^{b} K(w) d w=\int_{\mathbb{R}} K(w) d w
$$

Since for our kernel

$$
2 \int_{-\frac{1}{2} \pi}^{\frac{1}{2} \pi} K(w) d w=\int_{-\frac{1}{2} \pi}^{\frac{1}{2} \pi} \frac{4 \sin ^{2} \frac{1}{2} w}{\pi w^{2}} d w<1
$$

the constant $b$ determined by (10.9) will be larger than $\frac{1}{2} \pi$. However, one can use another kernel to show that in the theorem, it is sufficient to have $\lambda \delta>\frac{1}{2} \pi$; see Section 11.

Proof of Theorem 10.1. We know from Proposition 10.2 that $\beta=\sup |\sigma(u)|$ is finite and have to show that $\beta^{*}=\lim \sup |\sigma(u)|$ as $u \rightarrow \infty$ is equal to 0 . For given $\varepsilon^{\prime}>0$ we can change $\sigma$ on a finite interval $[0, B]$ in such a way that $\beta<\beta^{*}+\varepsilon^{\prime}$ for the new $\sigma$ while condition (i), (i') or (ii) is preserved. The new $\sigma$ and $F=\mathcal{L} \sigma$ will 
still satisfy the other conditions of the theorem. Since $\sigma$ is bounded and $F(x+i y)$ has good boundary behavior, one can let $x$ go to 0 in (10.5) to obtain the relation

$$
\left|\int_{\mathbb{R}} K_{\lambda}(v) \sigma(u+v) d v\right|=\left|\frac{1}{2 \pi} \int_{-\lambda}^{\lambda} \hat{K}_{\lambda}(y) F(i y) e^{i u y} d y\right| .
$$

Application of the Riemann-Lebesgue lemma then shows that

$$
M_{u}=\left|\int_{\mathbb{R}} K_{\lambda}(v) \sigma(u+v) d v\right|=o(1) \quad \text { as } u \rightarrow \infty .
$$

Proceeding as before we may now use (10.6) and (10.7) with $x=0$, replacing $M$ by $M_{u}, \beta_{x}$ by $\beta$ and $e^{-2 x \delta}=e^{-\eta}$ by 1 . Under each of the conditions (i), (i') and (ii) we can ensure (without undue increase in $\varepsilon$ 's) that $\lambda \delta$ is large enough so that (10.8) is satisfied. Taking $u$ large, one concludes that $\beta$ is small and, finally, that $\beta^{*}=0$.

Example 10.3. We will show that part (ii) in the proposition and theorem fails if $\lambda \delta<\frac{1}{2} \pi$. For an example we may change the scale to make $2 \delta=1$, after which we choose $\lambda<\pi$. Now consider the series

$$
\frac{1-e^{-z}}{\left(1+e^{-z}\right)^{2}}=\sum a_{n} e^{-n z}=1-3 e^{-z}+5 e^{-2 z}-7 e^{-3 z}+\ldots
$$

Then $\sigma(t)=\sum_{n \leq t} a_{n}$ is piecewise constant and the intervals of constancy have length $2 \delta=1$. For $z=x+i y$ with $x>0$ the sum of the series equals

$$
\int_{0-}^{\infty} e^{-z t} d \sigma(t)=z \int_{0}^{\infty} \sigma(t) e^{-z t} d t
$$

Thus

$$
F(z)=\mathcal{L} \sigma(z)=\frac{1-e^{-z}}{z} \frac{1}{\left(1+e^{-z}\right)^{2}} ;
$$

for $x \searrow 0$ and $-\lambda \leq y \leq \lambda$, the functions $F_{x}(i y)=F(x+i y)$ are uniformly convergent to a boundary function $F(i y)$. However, $\sigma$ is unbounded!

\section{An extremal problem}

We will show that one may take $b=\frac{1}{2} \pi$ in part (ii) of Theorem 10.1. For this we deal with the following

Problem 11.1. Determine the infimum $b^{*}$ of the constants $b>0$ for which there is an $L^{1}$ kernel $K=K_{1}$ on $\mathbb{R}$ with the following properties: $K$ is positive on a neighborhood of $[-b, b]$,

$$
2 \int_{-b}^{b} K(t) d t=\int_{\mathbb{R}}|K(t)| d t
$$

and the Fourier transform $\hat{K}$ has its support in $[-1,1]$. For convenience we also impose the normalization $\hat{K}(0)=1$.

Proposition 11.2. For $b=\frac{1}{2} \pi$ the conditions in Problem 11.1 are satisfied by the kernel $K$, given by

$$
K(t)=\frac{2 \cos t}{\pi^{2}-4 t^{2}}, \quad \hat{K}(y)= \begin{cases}\cos \frac{1}{2} \pi y & \text { for }|y| \leq 1 \\ 0 & \text { for }|y|>1\end{cases}
$$

No smaller constant will work; hence $b^{*}=\frac{1}{2} \pi$. 
That the function $\hat{K}$ in (11.2) is the Fourier transform of $K$ may be verified by Fourier inversion. With different scaling the kernel $K$ occurs in an article by Ingham [1936] on trigonometric inequalities and Dirichlet series. The author does not know if the extremal kernel is unique; cf. Section 20.

Proof of the proposition. (i) Let $K$ be as in (11.2). Then $K>0$ on $\left(-\frac{3}{2} \pi, \frac{3}{2} \pi\right)$ and $\hat{K}$ has support $[-1,1]$. We next verify (11.1) with $b=\frac{1}{2} \pi$. For the following computation, cf. Pitt 1958, section 4.3]. Consider the difference between the first and the second member of (11.1),

$$
\begin{aligned}
\Delta_{K} & =2 \int_{-\frac{1}{2} \pi}^{\frac{1}{2} \pi} K(t) d t-\int_{\mathbb{R}}|K(t)| d t \\
& =2 \int_{0}^{\frac{1}{2} \pi} K(t) d t-2 \int_{\frac{1}{2} \pi}^{\infty}|K(t)| d t=2 I_{0}-2 \sum_{n=0}^{\infty} J_{n}
\end{aligned}
$$

say, where the integration in $J_{n}$ is from $(4 n+1) \pi / 2$ to $(4 n+5) \pi / 2$. Then

$$
\begin{aligned}
I_{0} & =\int_{0}^{\frac{1}{2} \pi} K(t) d t=\frac{1}{\pi} \int_{0}^{1} \frac{\cos \frac{1}{2} \pi v}{1-v^{2}} d v \\
& =\frac{1}{2 \pi} \int_{0}^{1} \cos \frac{1}{2} \pi v\left(\frac{1}{1+v}+\frac{1}{1-v}\right) d v=\frac{1}{2 \pi} \int_{-1}^{1} \frac{\cos \frac{1}{2} \pi w}{w+1} d w \\
J_{n} & =\int_{(4 n+1) \pi / 2}^{(4 n+5) \pi / 2}|K(t)| d t=\frac{1}{\pi} \int_{4 n+1}^{4 n+5} \frac{\left|\cos \frac{1}{2} \pi v\right|}{v^{2}-1} d v \\
& =\frac{1}{2 \pi}\left(\int_{4 n+1}^{4 n+3}+\int_{4 n+3}^{4 n+5}\right)\left|\cos \frac{1}{2} \pi v\right|\left(\frac{1}{v-1}-\frac{1}{v+1}\right) d v \\
& =\frac{1}{2 \pi} \int_{-1}^{1} \cos \frac{1}{2} \pi w\left(\frac{1}{w+4 n+1}-\frac{1}{w+4 n+5}\right) d w
\end{aligned}
$$

Thus via a telescoping series under the integral sign,

$$
\Delta_{K}=\frac{1}{\pi} \int_{-1}^{1} \cos \frac{1}{2} \pi w\left\{\frac{1}{w+1}-\sum_{n=0}^{\infty}\left(\frac{1}{w+4 n+1}-\frac{1}{w+4 n+5}\right)\right\} d w=0
$$

This proves (11.1) for the kernel $K$ of (11.2) and $b=\frac{1}{2} \pi$. With this we have established the first part of Proposition 11.2.

(ii) We will verify that no constant $b<\frac{1}{2} \pi$ can work in Problem 11.1 after we make an application of the first part of Proposition 11.2.

Proposition 11.3. In part (ii) of Theorem 10.1 and Proposition 10.2 it is sufficient to take $\lambda \delta>\frac{1}{2} \pi$.

Proof. It will be enough to consider the case of Proposition 10.2. Observe that in its proof, the Fejér kernel $K_{\lambda}(v)$ may be replaced by any other kernel $K_{\lambda}(v)=\lambda K(\lambda v)$ such that $K$ has the properties listed in Problem 11.1. Indeed, $\hat{K}_{\lambda}$ will then have its support in $[-\lambda, \lambda]$ so that $(10.5)$ is valid for $K_{\lambda}$. We next fix $\lambda \delta$ just a little larger than $b$ so that $K_{\lambda}>0$ on $(-\delta, \delta)$. In (10.6) one now replaces

$$
\int_{|v|>\delta} K_{\lambda} \text { by } \int_{|v|>\delta}\left|K_{\lambda}\right|=\int_{\mathbb{R}}\left|K_{\lambda}\right|-\int_{-\delta}^{\delta} K_{\lambda}
$$


Thus (10.7) becomes

$$
M>\left(\left(1+e^{-\eta}\right) \int_{-\lambda \delta}^{\lambda \delta} K-\int_{\mathbb{R}}|K|\right) \beta_{x}-\varepsilon-\varepsilon^{\prime}
$$

For sufficiently small $\eta=2 \delta x$, the coefficient of $\beta_{x}$ will then be $>0$ by (11.1). In this way one obtains an upper bound for $\beta_{x}$ independent of (our small) $x$, and this bound will also work for $|\sigma|$. Hence, whenever $b$ is admissible in Problem 11.1, the piecewise constancy condition in Proposition 10.2 works as soon as $\lambda \delta>b$. (One can always decrease $\lambda$ or $\delta$ so that $\lambda \delta$ is only a little larger than $b$.)

We know that $b=\frac{1}{2} \pi$ works for the kernel $K$ of (11.2), so that the final part of Proposition 10.2 is valid for $\lambda \delta>\frac{1}{2} \pi$.

Proof of the second part of Proposition 11.2. Suppose that there would be a kernel $K$ which satisfies the conditions in Problem 11.1 with $b<\frac{1}{2} \pi$. In Example 10.3 (where $2 \delta=1$ ) we could then take $2 b<\lambda<\pi$ (so that $\lambda \delta>b$ ). The conclusion from the proof above would be that the function $\sigma$ in that example must be bounded - but it is not!

\section{Fatou and Riesz. General Dirichlet series}

Fatou's original theorem [1906, p. 389] may be stated as follows:

Theorem 12.1. Let $f(z)=\sum_{0}^{\infty} a_{n} z^{n}$ with $a_{n} \rightarrow 0$ so that $f$ is analytic on the unit disc. Suppose also that $f$ is analytic at the point $z_{0}=e^{i t_{0}} \in C(0,1)$, or more precisely, that $f$ has an analytic continuation (also called $f$ ) to a neighborhood of $z_{0}$. Then the series $\sum_{0}^{\infty} a_{n} z_{0}^{n}$ converges to $f\left(z_{0}\right)$.

Proofs. Marcel Riesz has given several proofs [1909, 1911, 1916] for Fatou's theorem; cf. Zeller and Beekmann [1970, p. 93]. The simplest proof goes as follows. One may take $z_{0}=1$ and choose $\lambda>0, R>1$ such that $f$ is analytic on the closed circular sector $V: 0 \leq|z| \leq R,-\lambda \leq \arg z \leq \lambda$. Setting $s_{n}(z)=\sum_{0}^{n} a_{k} z^{k}$, one considers the analytic functions

$$
g_{n}(z)=\frac{f(z)-s_{n}(z)}{z^{n+1}}\left(z-e^{i \lambda}\right)\left(z-e^{-i \lambda}\right), \quad z \in V .
$$

If one can prove that $g_{n}(z) \rightarrow 0$ uniformly on $\Gamma=\partial V$ as $n \rightarrow \infty$, the maximum principle will imply that $g_{n}(1) \rightarrow 0$ and hence $s_{n}(1) \rightarrow f(1)$. To prove the uniform convergence $g_{n}(z) \rightarrow 0$ on $\Gamma$, one considers different parts of $\Gamma$ separately. On the segments $z=r e^{ \pm i \lambda}$ with $0<r<1$, the smallness of the $a_{k}$ for $k>n$ together with one of the factors $z-e^{ \pm i \lambda}$ gives the desired result. On the segments $z=r e^{ \pm i \lambda}$ with $1<r<R$ and on the circular arc in $\Gamma$, one combines suitable bounds on the $a_{k}$ for $k \leq n$ with the effect of the denominator $z^{n+1}$ and the factors $z-e^{ \pm i \lambda}$. More details may be found in Landau and Gaier [1986, section 18].

Fatou himself had used Riemann's localization principle for trigonometric series $\mathcal{S}$ of the form $\sum_{-\infty}^{\infty} a_{n} e^{i n t}$ with $a_{n} \rightarrow 0$ as $|n| \rightarrow \infty$. Let $T$ be the generalized or distributional sum of the series:

$$
T=a_{0}+D^{2} \Phi, \quad \Phi(t)=-\sum_{n \neq 0} \frac{a_{n}}{n^{2}} e^{i n t} .
$$

[ $T$ is a so-called pseudofunction; cf. Section 14.] Suppose now that $T=g$ on $(\alpha, \beta)$, where $g$ is a periodic integrable function which is pointwise equal to the sum of its Fourier series $\sum_{-\infty}^{\infty} b_{n} e^{i n t}$. If one represents $g$ as $b_{0}+D^{2} G$ with continuous 
periodic $G$, the equality $T=g$ on $(\alpha, \beta)$ means that $a_{0}=b_{0}$ while $\Phi-G$ is linear on $(\alpha, \beta)$. By Riemann's theorem, the trigonometric series $\mathcal{S}$ converges to $g$ pointwise on $(\alpha, \beta)$; cf. Riemann [1892, pp. 227-271], Zygmund [1959, chapter 9, (5.7)]. To the function $f$ of Theorem 12.1 one can construct a periodic $C^{1}$ function $g$ such that $f\left(e^{i t}\right)=g(t)$ for $t$ in a neighborhood of $t_{0}$.

Another proof, based on W. H. Young's work 1918, may be found in Titchmarsh 1939, section 7.31]. It is of Fourier series type and (like Fatou's proof) can be used to obtain a more general result.

Fatou's theorem has been extended in many ways. Riesz considered general Dirichlet series and formulated conditions weaker than analyticity which imply convergence under appropriate coefficient conditions. Theorem 12.2 below contains notable classical extensions, including the striking part (ii) due to Ingham; cf. Remarks 12.3. For other extensions by Riesz and by Gaier, see Riesz [1924, Landau and Gaier [1986, section 12 and comments], Gaier [1953]. Newer results are discussed in Sections 13, 14.

Theorem 10.1 implies the following complex Tauberian theorem for Dirichlet series.

Theorem 12.2. Let $0=\mu_{0}<\mu_{1}<\mu_{2}<\ldots$ with $\mu_{n} \rightarrow \infty$ and let the series

$$
f(z)=\sum_{n=0}^{\infty} a_{n} e^{-\mu_{n} z}, \quad z=x+i y
$$

be absolutely convergent for $x>0$. Suppose that for some constant which we may call $f(0)$ and some number $\lambda>0$, the quotient

$$
F(z)=\frac{f(z)-f(0)}{z}, \quad z=x+i y, \quad x>0
$$

converges uniformly or in $L^{1}$ on $-\lambda \leq y \leq \lambda$ to a boundary function $F($ iy) as $x \searrow 0$. Then each of the following conditions is sufficient in order that $\sum_{0}^{\infty} a_{n}$ converges to $f(0)$ :

(i) the partial sums $s_{n}$ are 'very slowly decreasing':

$$
\liminf \sum_{u<\mu_{n} \leq t} a_{n} \geq 0 \quad \text { for } \quad u+1 \geq t \geq u \rightarrow \infty
$$

(no special condition required on $\lambda$ );

(ii) $\mu_{n+1}-\mu_{n} \geq 2 \delta>0$ for $n \geq n_{0}$ and $\lambda>\pi / 2 \delta$ (no special condition required on the coefficients $a_{n}$ ).

Proof. By changing $a_{0}$ one may assume $f(0)=0$. Define $\sigma(t)=\sum_{\mu_{n} \leq t} a_{n}$ and take $x>0$. By the absolute convergence of the series in (12.3) one may write

$$
f(z)=\int_{0-}^{\infty} e^{-z t} d \sigma(t)=z \int_{0}^{\infty} \sigma(t) e^{-z t} d t=z F(z),
$$

say (cf. Section 3). By the hypotheses the functions $\sigma$ and $F=\mathcal{L} \sigma$ will satisfy the conditions of Theorem 10.1, including one of the conditions (i') and (ii) for $\sigma$. Thus $\sigma(u) \rightarrow 0$ and this completes the proof.

Remarks 12.3. We first comment on part (i). There are related results in Ingham 1935, theorem 3(l)]. The present result which involves a one-sided Tauberian condition is an extension of the classical theorems of Fatou and Riesz. As observed already by Riesz, for convergence at $z=0$, the sum function $f(z)(12.3)$ need not 
be analytic there; weak regularity as in the theorem is sufficient. Fatou's theorem for power series corresponds to the case $\mu_{n}=n$. In his extension to Dirichlet series (12.3), Riesz [1916] gave a condition on the coefficients which implies 'very slow oscillation' of the partial sums $s_{n}$ as $n \rightarrow \infty$ :

$$
\lim \sum_{u<\mu_{n} \leq t} a_{n}=0 \text { for } u+1 \geq t \geq u \rightarrow \infty
$$

He also noted the sufficiency of the pair of conditions given by $a_{n}=o(1)$ plus $a_{n}=o\left(\mu_{n}-\mu_{n-1}\right)$. For the case of 'classical' Dirichlet series $\sum_{1}^{\infty} a_{n} / n^{z}$, Riesz explicitly mentioned the sufficient condition $n a_{n}=o(1)$. Theorem 12.2 implies the sufficiency of the condition $a_{n} \geq o(1)$ in Fatou's theorem and the condition $n a_{n} \geq o(1)$ in Riesz's theorem for classical Dirichlet series; cf. Korevaar [1954b] and Section 17 below. The one-sided condition for Fatou's theorem was also discussed in Postnikov [1979, section 17].

Part (ii) is in Ingham [1936]. In a later article [1950], Ingham observed that the inequality $\lambda>\pi / 2 \delta$ may be relaxed to $\lambda \geq \pi / 2 \delta$.

\section{Newer extensions of Fatou-Riesz}

Some newer complex Tauberian theorems have been motivated by operator theory; cf. Section 15. In that context Katznelson and Tzafriri [1986] obtained results for power series which contain Propositions 13.3 and 14.4. Proposition 13.3 was extended by Allan, O'Farrell and Ransford [1987 to Theorem 13.4 and by Arendt and Batty [1988 to Theorem 13.5. The formulations below are somewhat more general; cf. also Section 14 .

In this section $f$ stands for an analytic function on the unit disc:

$$
f(z)=\sum_{0}^{\infty} a_{n} z^{n} \quad \text { for }|z|<1 .
$$

In connection with Fatou's theorem we focus on conditions under which $a_{n} \rightarrow 0$ as $n \rightarrow \infty$. A simple condition is that $f$ be in the Hardy class $H^{1}$. This means that $f\left(r e^{i t}\right)$ converges to a boundary function $f\left(e^{i t}\right)$ in $L^{1}$ :

$$
\int_{-\pi}^{\pi}\left|f\left(r e^{i t}\right)-f\left(e^{i t}\right)\right| d t \rightarrow 0 \quad \text { as } r \nearrow 1
$$

see for example Duren [1970]. In this case Cauchy's formula and the RiemannLebesgue lemma imply that

$$
a_{n}=\frac{1}{2 \pi i} \int_{C(0, r)} f(z) z^{-n-1} d z=\frac{1}{2 \pi} \int_{-\pi}^{\pi} f\left(e^{i t}\right) e^{-i n t} d t \rightarrow 0 \quad \text { as } n \rightarrow \infty .
$$

It is convenient to introduce a local $H^{1}$ condition.

Definition 13.1. We say that $f(13.1)$ has $H^{1}$ boundary behavior at the point $z_{0}=e^{i t_{0}}$ if there is a number $\lambda>0$ such that $f\left(r e^{i t}\right)$ converges in $L^{1}\left(t_{0}-\lambda, t_{0}+\lambda\right)$ to a boundary function $f\left(e^{i t}\right)$ as $r \nearrow 1$.

Proposition 13.2. Let $f(z)=\sum_{0}^{\infty} a_{n} z^{n}$ be analytic and of class $H^{1}$ on the unit disc. Suppose that the quotient

$$
q(z)=\frac{f(z)-A}{1-z}=\sum_{0}^{\infty}\left(s_{n}-A\right) z^{n}, \quad \text { with } s_{n}=\sum_{0}^{n} a_{k},
$$


has $H^{1}$ boundary behavior at the point $z=1$. Then the series $\sum_{0}^{\infty} a_{n}$ converges to A.

Proof. Since $f$ is in $H^{1}$, the quotient $q$ has $H^{1}$ boundary behavior on the arcs $\left\{z=e^{i t}, \varepsilon \leq|t| \leq \pi\right\}$ with $\varepsilon>0$, where $1-z$ stays away from 0 . By the hypothesis $q$ also has $H^{1}$ boundary behavior on some arc with $-\lambda<t<\lambda$; hence $q$ is of class $H^{1}$ on the unit disc. By (13.2) applied to $q$ instead of $f$, the coefficients $s_{n}-A$ in the power series for $q$ tend to 0 as $n \rightarrow \infty$, so that $s_{n} \rightarrow A$.

Proposition 13.3. Let $f$ as in (13.1) have $H^{1}$ boundary behavior everywhere on the circle $C(0,1)$ except at the point $z=1$. Suppose that the sequence of partial sums $s_{n}=\sum_{0}^{n} a_{k}$ is bounded. Then $a_{n} \rightarrow 0$ as $n \rightarrow \infty$.

Proof. One may assume that $\left|s_{n}\right| \leq 1, \forall n$. Setting $s_{n}(z)=\sum_{0}^{n} a_{k} z^{k}$ and taking $0<\rho<1, \lambda>0$, one has

$$
a_{n}=\frac{1}{2 \pi i} \int_{C(0, \rho)} \frac{f(z)-s_{n-1}(z)}{z^{n+1}} \frac{\left(z-e^{i \lambda}\right)\left(z-e^{-i \lambda}\right)}{(z-1)^{2}} d z .
$$

Indeed, on the circle $C(0, \rho)$, the integrand $I(z)$ has the uniformly convergent expansion

$$
I(z)=\left(a_{n}+a_{n+1} z+\ldots\right)\left(1-e^{-i \lambda} z\right)\left(1-e^{i \lambda} z\right)(1+2 z+\ldots) / z .
$$

By Cauchy's theorem, the circle of integration may be changed to a new path $\Gamma^{\varepsilon}$ consisting of a short arc of the original circle from a point $\rho e^{-i \lambda}$ to $\rho e^{i \lambda}$, radial segments extending from the end points of this arc to a short distance $\varepsilon$ from $C(0,1)$, and a long arc of the circle $C(0,1-\varepsilon)$. Let $\rho=1-\lambda$ with small $\lambda \in\left(0, \frac{1}{2}\right)$. We will verify that one may actually let $\varepsilon$ go to 0 and replace $\Gamma^{\varepsilon}$ by the limit path $\Gamma=\sum_{1}^{4} \Gamma_{j}$ (Figure 3), where

$$
\left\{\begin{array}{l}
\Gamma_{1} \text { is the arc } z=\rho e^{i t} \text { with }-\lambda \leq t \leq \lambda, \\
\Gamma_{2} \text { is the segment } z=r e^{i \lambda} \text { with } \rho<r<1, \\
\Gamma_{3} \text { is the arc } z=e^{i t} \text { with } \lambda \leq t \leq 2 \pi-\lambda, \\
\Gamma_{4} \text { is the segment } z=r e^{-i \lambda} \text { with } 1>r>\rho .
\end{array}\right.
$$

For $|z|=r<1$ partial summation and the hypothesis $\left|s_{k}\right| \leq 1$ show that

$$
\left|\sum_{k=n}^{\infty} a_{k} z^{k}\right|=\left|\sum_{k=n}^{\infty} s_{k} z^{k}(1-z)-s_{n-1} z^{n}\right| \leq 2 \frac{r^{n}}{1-r}|1-z| .
$$

On the part $\Gamma \backslash \Gamma_{3}$ of $\Gamma$ inside the unit circle, simple considerations now give an upper bound for $|I(z)|$ independent of $\rho, \lambda, n$ :

$$
|I(z)| \leq \frac{2}{r} \frac{\left|r e^{i t}-e^{i \lambda}\right|\left|r e^{i t}-e^{-i \lambda}\right|}{(1-r)\left|1-r e^{i t}\right|}<\frac{4}{r}<10 .
$$

Because of this bound and the hypothesis that $f$ is in $H^{1}$ away from the point $z=1$, the auxiliary path of integration $\Gamma^{\varepsilon}$ may indeed be changed to $\Gamma$. It also follows that

$$
\left|\int_{\Gamma \backslash \Gamma_{3}} I(z) d z\right|<10 L\left(\Gamma \backslash \Gamma_{3}\right)<40 \lambda
$$




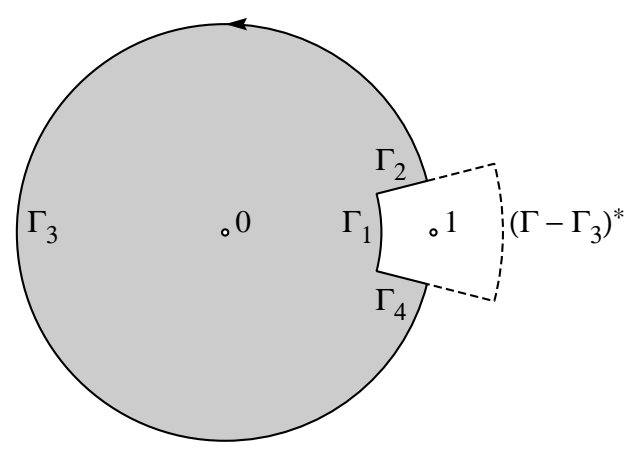

FiguRE 3. The paths of integration

In the integral over $\Gamma_{3}$ we treat $f$ and $s_{n-1}$ separately. For the case of $f$ one obtains the integral

$$
\int_{\lambda}^{2 \pi-\lambda} f\left(e^{i t}\right)\left(e^{i t}-e^{i \lambda}\right)\left(e^{i t}-e^{-i \lambda}\right)\left(e^{i t}-1\right)^{-2} e^{-i n t} i d t
$$

by the Riemann-Lebesgue lemma it tends to 0 as $n \rightarrow \infty$. To deal with the integral containing $s_{n-1}$, we observe that for $|z|>1$ the integrand can be written as

$$
\begin{aligned}
& J(z)=\frac{s_{n-1}(z)}{z^{n+1}} \frac{\left(z-e^{i \lambda}\right)\left(z-e^{-i \lambda}\right)}{(z-1)^{2}} \\
& =\left(a_{n-1}+a_{n-2} / z+\cdots+a_{0} / z^{n-1}\right)\left(1-e^{i \lambda} / z\right)\left(1-e^{-i \lambda} / z\right)(1+2 / z+\ldots) / z^{2} .
\end{aligned}
$$

Thus since $J$ is analytic outside the unit circle, the integral of $J$ over any circle $C(0, R)$ with $R>1$ is equal to 0 . Taking $R=1 / \rho$ we may change the circle of integration $C(0, R)$ here to the path $\Gamma^{*}$ which is obtained from $\Gamma$ by reflection in the unit circle. $\Gamma^{*}$ consists of $\Gamma_{3}$ plus the reflection $\left(\Gamma \backslash \Gamma_{3}\right)^{*}$ of $\Gamma \backslash \Gamma_{3}$; cf. Figure 3 . Thus the desired integral of $J$ over $\Gamma_{3}$ is equal to the negative of the integral of $J$ over $\left(\Gamma \backslash \Gamma_{3}\right)^{*}$. Now for $|z|=r>1$ one may use partial summation similar to (13.6) to get

$$
\left|\sum_{0}^{n-1} a_{k} z^{k}\right| \leq 2 \frac{r^{n}}{r-1}|z-1| .
$$

For $z$ lying on $\left(\Gamma \backslash \Gamma_{3}\right)^{*}$, so that $1 / z \in\left(\Gamma \backslash \Gamma_{3}\right)$, comparison with (13.7) shows that $|J(z)|<5$. By our definition of $\rho$ as $1-\lambda$, the length of $\left(\Gamma \backslash \Gamma_{3}\right)^{*}$ is at most twice the length of $\Gamma \backslash \Gamma_{3}$. The end result is that the integral of $J$ over $\left(\Gamma \backslash \Gamma_{3}\right)^{*}$, and hence the integral of $J$ over $\Gamma_{3}$, is majorized by $40 \lambda$.

For any given $\lambda \in\left(0, \frac{1}{2}\right]$ one may take $n_{0}$ so large that the integral (13.9) involving $f$ on $\Gamma_{3}$ is bounded by $20 \lambda$ for all $n \geq n_{0}$. Putting everything together one concludes that $\left|\int_{\Gamma_{3}} I(z) d z\right|<60 \lambda$ and

$$
\left|a_{n}\right|=\left|\frac{1}{2 \pi i} \int_{\Gamma} I(z) d z\right|<\frac{100}{2 \pi} \lambda \text { for all } n \geq n_{0} .
$$

Since $\lambda>0$ may be taken arbitrarily small, $a_{n} \rightarrow 0$ as $n \rightarrow \infty$.

Theorem 13.4. Let $f(z)=\sum_{0}^{\infty} a_{n} z^{n}$ be analytic for $|z|<1$ and let $E$ be the set of points $\zeta \in C(0,1)$ where $f$ is singular, in the sense that $f$ does not have $H^{1}$ 
boundary behavior at the points $\zeta$. Suppose that $E$ has linear measure 0 and that

$$
\sup _{\zeta \in E} \sup _{n \geq 0}\left|\sum_{0}^{n} a_{k} \zeta^{k}\right|=M<\infty .
$$

Then $a_{n} \rightarrow 0$ as $n \rightarrow \infty$. As a result, the series $\sum_{0}^{\infty} a_{n} z_{0}^{n}$ converges to $f\left(z_{0}\right)$ at every point $z_{0} \in C(0,1)$ where $f$ is weakly regular, in the sense that the quotient

$$
q(z)=\frac{f(z)-f\left(z_{0}\right)}{z-z_{0}}, \quad|z|<1
$$

has $H^{1}$ boundary behavior at the point $z_{0}$.

Proof (outline). The crucial observation is that the (closed) 'singularity set' $E \subset$ $C(0,1)$ can be enclosed in the union of a finite number of disjoint open arcs of arbitrarily small total length. One can then extend the complex method used for Proposition 13.3 (where $E=\{1\}$ ) to show that $a_{n} \rightarrow 0$. Knowing this, one may appeal to Theorem 12.2 for the convergence of the series $\sum_{0}^{\infty} a_{n} z_{0}^{n}$. For details, cf. the direct proof for the convergence of such series by Allan, O'Farrell and Ransford 1987. In Section 14 we will give a simpler proof with the aid of pseudofunctions, but the complex method is important for applications.

Arendt and Batty [1988] have proved an analog to Theorem 13.4 for Laplace transforms, which extends Theorem 7.1. In the formulation below we use the following terminology. Let $F$ be analytic in the half-plane $\{x=\operatorname{Re} z>0\}$. Then $F$ has $H^{1}$ boundary behavior at $z_{0}=i y_{0}$ if there is a number $\lambda>0$ such that $F(x+i y)$ converges to a boundary function $F(i y)$ in $L^{1}\left(y_{0}-\lambda<y<y_{0}+\lambda\right)$ as $x \searrow 0$.

Theorem 13.5. Let $\alpha(\cdot)$ be defined and bounded on $[0, \infty)$ so that the Laplace transform $F(z)=\mathcal{L} \alpha(z), z=x+i y$ is analytic for $x=\operatorname{Re} z>0$. Let $i E$ denote the set of all 'singular points' in of $F$ on $i \mathbb{R}$, in the sense that $F$ does not have $H^{1}$ boundary behavior at the points i $\eta$. Suppose that $i E$ has linear measure zero and that

$$
\sup _{\eta \in E} \sup _{B>0}\left|\int_{0}^{B} \alpha(t) e^{-i \eta t} d t\right| \leq M<\infty .
$$

Then

$$
\int_{0}^{\infty} \alpha(t) e^{-z_{0} t} d t=F\left(z_{0}\right)
$$

at all points $z_{0}=i y_{0}$ where $F$ is weakly regular, in the sense that the quotient

$$
Q(z)=\frac{F(z)-F\left(z_{0}\right)}{z-z_{0}}, \quad \operatorname{Re} z>0
$$

has $H^{1}$ boundary behavior at the point $z_{0}$.

Remarks 13.6. The original work of Fatou and Riesz was refined by Ingham [1935. Newman's contour integration method, as described in Section 7, has played a role in recent developments. There are now extensions, related results and applications to operator theory by many authors. Besides the papers referred to already one may mention Ransford [1988], Batty [1990], [1994a], Arendt and Prüss [1992], Arendt and Batty [1995], Batty, van Neerven and Räbiger [1998], and Chill [1998]. For 
comprehensive treatment of the area, see the book by Arendt, Batty, Hieber and Neubrander [2001].

\section{Pseudofunction Boundary Behavior}

The results in Section 13 may be refined with the aid of the distributional approach initiated by Katznelson and Tzafriri [1986]. As before, let

$$
f(z)=\sum_{0}^{\infty} a_{n} z^{n} \quad \text { for }|z|<1 .
$$

We continue our discussion of conditions under which $a_{n} \rightarrow 0$. Instead of convergence in $L^{1}$ we consider distributional convergence of $f\left(r e^{i t}\right)$ to a boundary distribution $T=f\left(e^{i t}\right)$. That is,

$$
\int_{-\pi}^{\pi} f\left(r e^{i t}\right) \phi(t) d t \rightarrow<T, \phi>=\int_{-\pi}^{\pi} f\left(e^{i t}\right) \phi(t) d t, \quad \text { as } r \nearrow 1
$$

for all $C^{\infty}$ functions $\phi$ of period $2 \pi$, the 'testing functions' for periodic distributions.

In the class $C_{2 \pi}^{\infty}$ of testing functions, convergence $\phi_{j} \rightarrow \phi$ is defined by the requirement that $\phi_{j} \rightarrow \phi$ uniformly, $\phi_{j}^{\prime} \rightarrow \phi^{\prime}$ uniformly, $\phi_{j}^{\prime \prime} \rightarrow \phi^{\prime \prime}$ uniformly, etc. Thus $C_{2 \pi}^{\infty}$ becomes the testing space $\mathcal{D}_{2 \pi}$. A distribution $T$ of period $2 \pi$ is a continuous linear functional on $\mathcal{D}_{2 \pi}:\left\langle T, \phi_{j}>\rightarrow<T, \phi>\right.$ whenever $\phi_{j} \rightarrow \phi$ in $\mathcal{D}_{2 \pi}$. With convergence $T_{k} \rightarrow T$ given by the relation $\left\langle T_{k}, \phi\right\rangle \rightarrow\langle T, \phi\rangle$ for all $\phi$, the periodic distributions form the dual space $\mathcal{D}_{2 \pi}^{\prime}$ of $\mathcal{D}_{2 \pi}$.

The Fourier series of a periodic distribution $T$ is defined as

$$
\sum_{-\infty}^{\infty} c_{n}[T] e^{i n t} \quad \text { with } c_{n}[T]=\frac{1}{2 \pi}<T, e^{-i n t}>
$$

The Fourier coefficients $c_{n}[\phi]$ of a testing function $\phi$ are $\mathcal{O}\left(|n|^{-p}\right)$ as $|n| \rightarrow \infty$ for every $p$. Thus a trigonometric series $\sum_{-\infty}^{\infty} b_{n} e^{i n t}$ is convergent in $\mathcal{D}_{2 \pi}^{\prime}$ if (and only if) $b_{n}=\mathcal{O}\left(|n|^{q}\right)$ for some constant $q$. Since the Fourier series of a testing function $\phi$ converges to $\phi$ in $\mathcal{D}_{2 \pi}$, the Fourier series of $T$ converges to $T$ in $\mathcal{D}_{2 \pi}^{\prime}$.

Distributions $T$ are multiplied by testing functions $\omega$ according to the rule $<T \omega, \phi>=\langle T, \omega \phi>$. For the Fourier coefficients this means that

$$
c_{n}[T \omega]=\sum_{k} c_{k}[\omega] c_{n-k}[T] .
$$

Definition 14.1. A periodic distribution $T$ whose Fourier coefficients $b_{n}=c_{n}[T]$ form a bounded sequence is called a pseudomeasure. If $b_{n} \rightarrow 0$ as $n \rightarrow \pm \infty$, one speaks of a pseudofunction.

The product of a pseudomeasure or pseudofunction $T$ and a testing function $\omega$ is again a pseudomeasure or pseudofunction, respectively. This follows from (14.3):

$$
\left|c_{n}[T \omega]\right| \leq \sum_{|k| \leq B}\left|c_{k}[\omega]\right| \sup _{|j| \geq|n|-B}\left|c_{j}[T]\right|+\sup _{j}\left|c_{j}[T]\right| \sum_{|k|>B}\left|c_{k}[\omega]\right| ;
$$

if $c_{n}[T] \rightarrow 0$ as $n \rightarrow \pm \infty$, then also $c_{n}[T \omega] \rightarrow 0$. These results are also true for certain functions $\omega$ that are less smooth than testing functions. Indeed, one may use formula (14.3) to define the product $T \omega$. In the case of pseudomeasures and pseudofunctions it is then enough to require that $\omega$ have an absolutely convergent 
Fourier series. Useful functions of the latter kind are the periodic trapezoidal functions $\tau_{\lambda}$ with $\lambda \leq \frac{1}{2} \pi$ which for $|t| \leq \pi$ are given by

$$
\tau_{\lambda}(t)= \begin{cases}1 & \text { for }|t| \leq \lambda, \\ 0 & \text { for } 2 \lambda \leq|t| \leq \pi, \\ 2-|t| / \lambda & \text { for } \lambda \leq|t| \leq 2 \lambda\end{cases}
$$

By a short calculation

$$
c_{n}\left[\tau_{\lambda}\right]=\frac{\cos \lambda n-\cos 2 \lambda n}{\pi \lambda n^{2}} .
$$

One sometimes needs other trapezoidal functions. We will speak of a trapezoidal testing function $\tau_{\lambda}$ if $\tau_{\lambda}$ is in $C_{2 \pi}^{\infty}$ and satisfies the first two lines in (14.4).

If the analytic function $f(z)=\sum_{0}^{\infty} a_{n} z^{n}$ on the unit disc has a boundary distribution $T=f\left(e^{i t}\right)$, it follows from (14.2) with $\phi(t)=e^{-i n t}$ that $f\left(e^{i t}\right)$ has Fourier coefficients equal to $a_{n}$ for $n \geq 0$ and equal to 0 for $n<0$.

We now turn to local boundary behavior.

Definition 14.2. We will say that the function $f$ in (14.1) has pseudofunction boundary behavior at the point $z_{0}=e^{i t_{0}}$ if $f\left(r e^{i t}\right)$ has a boundary distribution $f\left(e^{i t}\right)$ on $C(0,1)$ which on some interval $\left(t_{0}-\lambda, t_{0}+\lambda\right)$ coincides with a pseudofunction $g(t)$.

If $f$ has pseudofunction boundary behavior at every point of the circle $C(0,1)$, $f\left(e^{i t}\right)$ is a pseudofunction. This may be proved with the aid of a suitable 'partition of unity': one may represent the constant function 1 as a sum of trapezoidal functions with small support.

Proposition 14.3. Let $f(z)=\sum_{0}^{\infty} a_{n} z^{n}$ be analytic on the unit disc and have a pseudofunction boundary distribution $F(t)=f\left(e^{i t}\right)$ on the circle $C(0,1)$. Suppose that the quotient

$$
q(z)=\frac{f(z)-A}{1-z}=\sum_{0}^{\infty}\left(s_{n}-A\right) z^{n}, \quad \text { with } s_{n}=\sum_{0}^{n} a_{k},
$$

has pseudofunction boundary behavior at the point $z=1$. Then the series $\sum_{0}^{\infty} a_{n}$ converges to $A$.

Proof. The hypotheses imply that $a_{n}=o(1)$; hence $s_{n}-A=o(|n|)$ as $|n| \rightarrow \infty$. It follows that $q\left(r e^{i t}\right)=\sum_{0}^{\infty}\left(s_{n}-A\right) r^{n} e^{i n t}$ tends to the boundary distribution $Q(t)=q\left(e^{i t}\right)=\sum_{0}^{\infty}\left(s_{n}-A\right) e^{i n t}$ as $r \nearrow 1$. By the hypotheses $Q$ is equal to a pseudofunction $G$ on some interval $(-\mu, \mu)$. As a result one has

$$
Q \tau_{\lambda}=G \tau_{\lambda}, \text { a pseudofunction, }
$$

whenever $\tau_{\lambda}$ is a trapezoidal testing function with support in $(-\mu, \mu)$. Indeed, for any testing function $\phi$,

$$
<Q \tau_{\lambda}, \phi>=<Q, \tau_{\lambda} \phi>=<G, \tau_{\lambda} \phi>=<G \tau_{\lambda}, \phi>.
$$

We will show that away from the point $t=0, Q(t)$ is equal to a pseudofunction $H(t)$, with the symbolic form $\left\{f\left(e^{i t}\right)-A\right\} /\left(1-e^{i t}\right)$. To that end we consider the product of

$$
T_{r}(t)=f\left(r e^{i t}\right)-A \quad \text { and } \quad \omega_{r}(t)=\frac{1}{1-r e^{i t}}\left\{1-\tau_{\lambda}(t)\right\}
$$


where we let $r$ run over the interval $(0,1) . T_{r}$ is a pseudofunction; its Fourier coefficients $c_{n}\left[T_{r}\right]$ are dominated by $\left|a_{n}\right|$ if $n>0\left(\left|a_{0}-A\right|\right.$ if $\left.n=0\right)$ and they vanish for $n<0$. Hence $c_{n}\left[T_{r}\right]=o(1)$ as $|n| \rightarrow \infty$, uniformly in $r$. The factor $\omega_{r}$ is a testing function with support $(-\pi,-\lambda] \cup[\lambda, \pi](\bmod 2 \pi)$. Its second derivative $\omega_{r}^{\prime \prime}$ is bounded by a constant independent of $r$. Integration by parts thus shows that the Fourier coefficients $c_{n}\left[\omega_{r}\right]$ are majorized by $C /\left(n^{2}+1\right)$, with $C$ independent of $r$. One now derives from formula (14.3) that

$$
\begin{gathered}
\left|c_{n}\left[T_{r} \omega_{r}\right]\right| \leq \sum_{k}\left|c_{k}\left[T_{r}\right]\right|\left|c_{n-k}\left[\omega_{r}\right]\right| \leq A_{n}, \quad \text { where } \\
A_{n}=C\left\{\frac{\left|a_{0}-A\right|}{n^{2}+1}+\sum_{k=1}^{\infty} \frac{\left|a_{k}\right|}{(n-k)^{2}+1}\right\} \rightarrow 0 \quad \text { as }|n| \rightarrow \infty .
\end{gathered}
$$

Since

$$
T_{r}(t) \omega_{r}(t)=q\left(r e^{i t}\right)\left\{1-\tau_{\lambda}(t)\right\} \rightarrow Q(t)\left\{1-\tau_{\lambda}(t)\right\} \quad \text { as } r \nearrow 1
$$

the Fourier coefficients $c_{n}$ of the limit distribution are also majorized by $A_{n}=o(1)$ as $|n| \rightarrow \infty$. Hence

$$
Q\left(1-\tau_{\lambda}\right)=\text { a pseudofunction } H \text {. }
$$

Combining (14.7) and (14.8), one finds that for small $\lambda>0$,

$$
Q=Q \tau_{\lambda}+Q\left(1-\tau_{\lambda}\right)=G \tau_{\lambda}+H .
$$

The sum on the right is a pseudofunction, hence the Fourier coefficients $s_{n}-A$ of $Q(t)=q\left(e^{i t}\right)$ tend to 0 as $n \rightarrow \infty$. This observation completes the proof.

Proposition 14.4. Let $f(z)=\sum_{0}^{\infty} a_{n} z^{n}$ as in (14.1) have pseudofunction boundary behavior everywhere on the circle $C(0,1)$ except perhaps at the point $z=1$. Suppose that the sequence of partial sums $s_{n}=\sum_{0}^{n} a_{k}$ is bounded. Then $a_{n} \rightarrow 0$ as $n \rightarrow \infty$.

Proof. Since $\left|s_{n}\right| \leq M<\infty, \forall n$, the quotient

$$
q(z)=\frac{f(z)}{1-z}=\sum_{0}^{\infty} s_{n} z^{n}
$$

has boundary pseudomeasure $Q(t)=\sum_{0}^{\infty} s_{n} e^{i n t}$ on $C(0,1)$. The sequence $\left\{a_{n}\right\}=$ $\left\{s_{n}-s_{n-1}\right\}$ is also bounded; hence $f(z)$ has boundary pseudomeasure $F(t)=$ $\sum_{0}^{\infty} a_{n} e^{i n t}$. By the hypotheses $F$ is equal to a pseudofunction $G$ away from the point $t=0$. Thus for any trapezoidal function $\tau_{\lambda}$ as in (14.4),

$$
F\left(1-\tau_{\lambda}\right)=G\left(1-\tau_{\lambda}\right)=H_{\lambda}, \text { a pseudofunction. }
$$

On the other hand $f(z)=(1-z) q(z)$, so that $F(t)=\left(1-e^{i t}\right) Q(t)$ and

$$
F(t) \tau_{\lambda}(t)=Q(t)\left(1-e^{i t}\right) \sum_{k} c_{k}\left[\tau_{\lambda}\right] e^{i k t}=Q(t) \sum_{k}\left\{c_{k}\left[\tau_{\lambda}\right]-c_{k-1}\left[\tau_{\lambda}\right]\right\} e^{i k t}
$$

We proceed to estimate the Fourier coefficients of the pseudomeasure $F \tau_{\lambda}$. By (14.10), (14.3) and the fact that $\left|c_{j}[Q]\right|=\left|s_{j}\right| \leq M$,

$$
\left|c_{n}\left[F \tau_{\lambda}\right]\right| \leq \sum_{k}\left|c_{n-k}[Q]\right|\left|c_{k}\left[\tau_{\lambda}\right]-c_{k-1}\left[\tau_{\lambda}\right]\right| \leq M \sum_{k}\left|c_{k}\left[\tau_{\lambda}\right]-c_{k-1}\left[\tau_{\lambda}\right]\right| .
$$


Formula (14.5) shows that the final sum is majorized by

$$
\begin{aligned}
& \sum_{k} \frac{\lambda}{\pi}\left|\frac{\cos k \lambda-\cos 2 k \lambda}{k^{2} \lambda^{2}}-\frac{\cos (k-1) \lambda-\cos 2(k-1) \lambda}{(k-1)^{2} \lambda^{2}}\right| \\
& =\frac{\lambda}{\pi} \sum_{k}\left|\int_{(k-1) \lambda}^{k \lambda} \frac{d}{d x} \frac{\cos x-\cos 2 x}{x^{2}} d x\right| \\
& \leq \frac{\lambda}{\pi} \int_{\mathbb{R}}\left|\frac{d}{d x} \frac{\cos x-\cos 2 x}{x^{2}}\right| d x=C \lambda .
\end{aligned}
$$

Combining (14.9) - (14.12) one obtains the inequality

$$
\left|a_{n}\right|=\left|c_{n}[F]\right|=\left|c_{n}\left[F\left(1-\tau_{\lambda}\right)\right]+c_{n}\left[F \tau_{\lambda}\right]\right| \leq\left|c_{n}\left[H_{\lambda}\right]\right|+M C \lambda .
$$

Since $H_{\lambda}$ is a pseudofunction, it follows that $\left|a_{n}\right| \leq 2 M C \lambda$ for $n \geq n_{0}(\lambda)$. Now $\lambda>0$ may be taken arbitrarily small; hence $a_{n} \rightarrow 0$ as $n \rightarrow \infty$.

We can now prove the result in Theorem 13.4 under weaker conditions.

Theorem 14.5. Let $f(z)=\sum_{0}^{\infty} a_{n} z^{n}$ be analytic for $|z|<1$ and have pseudofunction boundary behavior at all points $\zeta \in C(0,1)$ outside a subset $E \subset C(0,1)$. Suppose that $E$ has linear measure 0 and that

$$
\sup _{\zeta \in E} \sup _{n \geq 0}\left|\sum_{0}^{n} a_{k} \zeta^{k}\right|=M<\infty
$$

Then $a_{n} \rightarrow 0$ as $n \rightarrow \infty$ (so that $E$ is empty). As a result, the series $\sum_{0}^{\infty} a_{n} z_{0}^{n}$ converges to $f\left(z_{0}\right)$ at every point $z_{0} \in C(0,1)$ where $f$ is weakly regular, in the sense that the quotient

$$
q(z)=\frac{f(z)-f\left(z_{0}\right)}{z-z_{0}}, \quad|z|<1
$$

has pseudofunction boundary behavior at the point $z_{0}$.

Proof. In view of Proposition 14.3 it is enough to show that $a_{n} \rightarrow 0$. If $E$ is empty, $f(z)$ has boundary pseudofunction $f\left(e^{i t}\right)$ on $C(0,1)$ so that $a_{n} \rightarrow 0$. We may thus assume that $E$ is not empty or there is nothing to prove. Then it follows from (14.14) that the sequence $\left\{a_{n}\right\}$ is bounded, so that $f(z)$ has boundary pseudomeasure $F(t)=f\left(e^{i t}\right)$.

The complement of the compact set $E$ on the unit circumference has measure $2 \pi$. It can be represented as the union of a countable family of maximal open arcs. One may choose a finite number of these arcs of total length very close to $2 \pi$. On each of these $\operatorname{arcs} F$ is equal to a pseudofunction. The finitely many complementary closed arcs jointly cover $E$. It is convenient to replace degenerate arcs among them by closed arcs of very small positive length. Let $l$ be the minimal length of the arcs in our covering. To prepare for an argument similar to that used for Proposition 14.4, we extend each of the closed arcs by a closed arc of length $l$ at both ends. Combining arcs with nonempty intersection we obtain a covering of $E$ by a finite set of disjoint closed $\operatorname{arcs} J_{1}, \ldots, J_{p}$. It may be assumed that their total length is bounded by a preassigned number $\varepsilon>0$.

Let us focus on one of the $\operatorname{arcs} I=J_{k}$ and identify the point $e^{i t}$ of the circle with $t(\bmod 2 \pi)$. Then $I$ becomes an interval $a-l \leq t \leq b+l$ such that $[a, b]$ intersects $E$ while $[a-l, a)$ and $(b, b+l]$ do not. We now introduce a partition of unity $\mathcal{P}$ on 'the circle' $(-\pi, \pi]$, consisting of $N$ translates $\tau_{\lambda}^{*}$ of the elementary trapezoidal 
function $\tau_{\lambda}$ in (14.4). Observe that the support of $\tau_{\lambda}^{*}$ has length $4 \lambda$, that the sum of two consecutive functions $\tau_{\lambda}^{*}$ has support of length $7 \lambda$, and that $2 \pi$ must be $N$ times $3 \lambda$. We will take $\lambda<l / 7$, so that every open interval of length $l$ contains the support of one of the functions in $\mathcal{P}$. Taking $N=2 \pi /(3 \lambda)$ minimal one finds that $\lambda \geq l / 8$. A picture indicates that the number of functions $\tau_{\lambda}^{*}$ whose support intersects $[a, b]$ is majorized by $(b-a) / \lambda$. Thus the total number of $\tau_{\lambda}^{*} \in \mathcal{P}$ whose support meets $E$ is majorized by $\sum_{k} L\left(J_{k}\right) / \lambda \leq \varepsilon / \lambda$.

For a function $\tau_{\lambda}^{*}$ whose support meets $E$, a computation similar to the one in $(14.10)-(14.12)$ will show that the Fourier coefficients $c_{n}\left[F \tau_{\lambda}^{*}\right]$ are majorized by $M C^{\prime} \lambda$, where $M$ is as in (14.14) and $C^{\prime}$ is an absolute constant. We sketch the details. In the earlier proof, the trapezoidal function $\tau_{\lambda}$ was centered at the singular point $t=0$. In the present case, the 'center' of $\tau_{\lambda}^{*}$ may have distance $\mu$ to $E$, where $\mu \leq 2 \lambda$. This corresponds to the situation in the earlier proof in which $\tau_{\lambda}$ would be shifted over a distance $\mu$. The effect would be that the Fourier coefficients $c_{n}\left[\tau_{\lambda}\right]$ are multiplied by $e^{i n \mu}$ or $e^{-i n \mu}$, say the first. In this situation

$$
\begin{aligned}
& \sum_{k}\left|c_{k}-c_{k-1}\right| \leq \frac{\lambda}{\pi} \int_{\mathbb{R}}\left|\frac{d}{d x}\left(\frac{\cos x-\cos 2 x}{x^{2}} e^{i(\mu / \lambda) x}\right)\right| d x \\
& \leq \frac{\lambda}{\pi} \int_{\mathbb{R}}\left\{\frac{\mu}{\lambda}\left|\frac{\cos x-\cos 2 x}{x^{2}}\right|+\left|\frac{d}{d x} \frac{\cos x-\cos 2 x}{x^{2}}\right|\right\} d x \leq C^{\prime} \lambda .
\end{aligned}
$$

Here $C^{\prime}$ corresponds to the worst case $|\mu / \lambda|=2$. It follows that $\left|c_{n}\left[F \tau_{\lambda}^{*}\right]\right| \leq M C^{\prime} \lambda$ whenever the support of $\tau_{\lambda}^{*}$ meets $E$.

We now consider all functions $\tau_{\lambda}^{*} \in \mathcal{P}$ whose support meets $E$. Multiplying their number by the above bound on $\left|c_{n}\right|$, we find that the sum of the Fourier coefficients $c_{n}\left[F \tau_{\lambda}^{*}\right]$ with $\tau_{\lambda}^{*} \in \mathcal{P}$ has absolute value $\leq(\varepsilon / \lambda) M C^{\prime} \lambda=M C^{\prime} \varepsilon$. For the finitely many $\tau_{\lambda}^{*} \in \mathcal{P}$ whose support does not meet $E$, the product $F \tau_{\lambda}^{*}$ is a pseudofunction; the sum of the corresponding $c_{n}$ tends to 0 as $|n|$ goes to $\infty$. The final conclusion is that $a_{n} \rightarrow 0$; cf. (14.13).

The second part of the theorem now follows from Proposition 14.3.

We do not formulate the corresponding general result for Laplace transforms, but describe how one can strengthen Theorem 7.1 by the introduction of pseudofunction boundary behavior.

Theorem 14.6. Let $\alpha(\cdot)$ vanish on $(-\infty, 0)$ and be bounded on $[0, \infty)$, so that the Laplace transform $F(z)=\mathcal{L} \alpha(z), z=x+i y$ is analytic for $x=\operatorname{Re} z>0$. Suppose that $F(x+i y)$ has pseudofunction boundary behavior as $x \searrow 0$ and that $F$ is weakly regular at the origin, in the sense that for some constant which may be called $F(0)$, the quotient

$$
Q(x+i y)=\frac{F(x+i y)-F(0)}{x+i y}, \quad x>0
$$

has pseudofunction boundary behavior at the origin. Then

$$
\int_{0}^{\infty} \alpha(t) d t=F(0)
$$

Locally integrable functions $G_{x}(i y)=G(x+i y)$ on $-\infty<y<\infty$ converge distributionally to $G(i y)$ as $x \searrow 0$ if

$$
\int_{\mathbb{R}} G(x+i y) \phi(y) d y \rightarrow<G(i y), \phi(y)>
$$


for all testing functions $\phi$, usually the $C^{\infty}$ functions with compact support. The limit distribution $G(i y)$ is locally equal to a pseudofunction if the Fourier coefficients $c_{n}$ of the products $G(i y) \phi(y)$ on an appropriate interval tend to zero as $|n| \rightarrow \infty$. Replacing $\phi(y)$ by products $\phi(y) e^{i \delta y}$ with $\delta \in[0, a)$, one may verify the equivalent statement that $<G(i y), \phi(y) e^{i b y}>\rightarrow 0$ as $|b| \rightarrow \infty$. For bounded $\alpha(\cdot)$ with support on $\mathbb{R}^{+}$, pseudofunction boundary behavior of $F(x+i y)=\mathcal{L} \alpha(x+i y)$ turns out to be equivalent to the limit relation

$$
\int_{u}^{u+h} \alpha(t) d t \rightarrow 0 \quad \text { as } u \rightarrow \infty, \forall h>0 .
$$

Proof of Theorem 14.6 (outline). As in Section 7 we may take $F(0)=0$ and $\sup |\alpha(t)| \leq 1$. This time it is convenient to use a rectangular contour in the right half-plane instead of the semicircle $\Gamma_{1}$. The estimate $|F(x+i y)| \leq 1 / x$ and Cauchy's theorem now imply that the integral

$$
I(B, x)=\int_{-R}^{R} F(x+i y)\left(\frac{1}{x+i y}+\frac{x+i y}{R^{2}}\right) e^{B(x+i y)} d y
$$

tends to a limit $I(B)$ as $x \searrow 0$. One wants to show that $I(B)$ is small when $B$ is large.

Let $\chi^{J}$ denote the characteristic function of the interval $J$ and let $\tau_{\mu}$ be a trapezoidal function which is equal to 1 on $[-\mu, \mu]$ and equal to 0 outside a neighborhood of $[-\mu, \mu]$. Setting

$$
G(x+i y)=F(x+i y)\left(\frac{1}{x+i y}+\frac{x+i y}{R^{2}}\right) e^{B x} \chi^{[-R, R]}(y),
$$

one can write the integral (14.15) in distributional notation as

$$
I(B, x)=<G(x+i y), e^{i B y} \tau_{R}(y)>.
$$

One may now employ the method used for Proposition 14.3 to show that $G(x+i y)$ tends to a pseudofunction $G(i y)$ as $x \searrow 0$. To prove such convergence, one would separately consider

$$
G(x+i y) \tau_{\lambda}(y) \text { and } G(x+i y)\left\{1-\tau_{\lambda}(y)\right\} \tau_{R}(y)
$$

on a suitable open interval containing $[-R, R]$. The hypotheses imply that for small $\lambda>0$, the first expression converges to a pseudofunction $G(i y) \tau_{\lambda}(y)$. For the second expression one may consider the product of

$$
F(x+i y) e^{B x} \tau_{R}(y) \text { and }\left(\frac{1}{x+i y}+\frac{x+i y}{R^{2}}\right) \chi^{[-R, R]}(y)\left\{1-\tau_{\lambda}(y)\right\} .
$$

The Fourier coefficients $c_{n}$ of the first factor are $o(1)$ as $|n| \rightarrow \infty$, uniformly for $0<x<1$, while the coefficients $c_{n}$ of the second factor are $\mathcal{O}\left\{1 /\left(n^{2}+1\right)\right\}$, uniformly in $x$. Hence the second expression in (14.18) also converges to a pseudofunction.

By the above there is then a pseudofunction $G(i y)$ such that the limit of $I(B, x)$ as $x \searrow 0$ has the form $I(B)=<G(i y), e^{i B y} \tau_{R}(y)>$. That limit tends to zero as $B \rightarrow \infty$.

Remarks 14.7. One may also introduce pseudofunction boundary behavior in the statements of the Wiener-Ikehara theorem and Proposition 4.3. Furthermore, the method of Proposition 14.3 can be used to derive a 'pseudofunction form' of Riemann's localization principle. 


\section{Applications to operator theory}

The results in Sections 13, 14 have analogs for analytic functions with values in a Banach space, including a space of bounded linear operators. The proofs are similar to those for the scalar case. For details we refer to the papers and the book quoted in Section 13.

For the discussion of applications we recall some definitions. Let $L$ be a bounded linear operator $L$ on a (complex) Banach space $X$. The resolvent set of $L$ is the (open) set of complex numbers $\lambda$ for which $L-\lambda I$ has a bounded inverse. The spectrum of $L, \operatorname{sp}(L)$, is defined as the complement of the resolvent set. There are names for special subsets of $\operatorname{sp}(L)$. The numbers $\lambda$ for which $L-\lambda I$ fails to be one to one form the point spectrum. The continuous spectrum consists of those $\lambda$ for which $L-\lambda I$ is one to one onto a dense subspace of $X$. If the range of $L-\lambda I$ fails to be dense in $X$, one says that $\lambda$ belongs to the residual spectrum of $L$. It coincides with the point spectrum of the adjoint $L^{*}$ of $L$ and may overlap the point spectrum of $L$.

Here we consider so-called power bounded operators $L$ on $X$, that is,

$$
\sup _{n \geq 0}\left\|L^{n}\right\|=M<\infty .
$$

In this case $I-L / \lambda$ has a bounded inverse whenever $|\lambda|>1$, given by the usual geometric series. Hence the spectrum belongs to the closed unit disc. For the following we need only the 'peripheral spectrum', the part of $\operatorname{sp}(L)$ on the circumference $C(0,1)$.

Work of Esterle [1983, section 9] and Katznelson and Tzafriri [1986] implies

Proposition 15.1. Let $L$ be a power bounded linear operator on $X$ whose spectrum meets $C(0,1)$ only in the point $z=1$. Then

$$
\left\|L^{n}-L^{n+1}\right\| \rightarrow 0 \text { as } n \rightarrow \infty .
$$

Proof. The resolvent set of $L$ contains the exterior of the unit disc and the circle $C(0,1)$ except for the point $z=1$. Thus the operator $I-z L$ is invertible for $|z| \leq 1$ except when $z=1$. For $|z|<1$ its inverse is given by the operator-valued function

$$
g(z)=\sum_{0}^{\infty} s_{n} z^{n} \stackrel{\text { def }}{=} \sum_{0}^{\infty} L^{n} z^{n} .
$$

This function is analytic for $|z|<1$ as well as in a neighborhood of each point $\zeta \in C(0,1) \backslash 1$. The same is true for

$$
f(z)=(1-z) g(z)=\sum_{0}^{\infty} a_{n} z^{n}=I+\sum_{1}^{\infty}\left(L^{n}-L^{n-1}\right) z^{n} .
$$

Now the sequence $\left\{s_{n}\right\}$ is bounded; hence by an analog of Proposition 13.3 for operator-valued functions, $\left\|a_{n}\right\|=\left\|L^{n}-L^{n-1}\right\| \rightarrow 0$. This proves the result.

As a corollary one obtains a classical result of Gel'fand [1941]:

Corollary 15.2. If $L$ is an isometry or, more generally, if

$$
\sup _{n \in \mathbb{Z}}\left\|L^{n}\right\|=M<\infty,
$$

and if $\operatorname{sp}(L)=\{1\}$, then $L$ is the identity. 
Indeed, for given $\varepsilon>0$ and large $n>0,\left\|L^{n}-L^{n+1}\right\|<\varepsilon$; hence

$$
\|I-L\|=\left\|L^{-n}\left(L^{n}-L^{n+1}\right)\right\| \leq M \varepsilon .
$$

The method of Proposition 15.1 also gives

Proposition 15.3. Let $L$ be a power bounded operator on $X$ (15.1) such that $\operatorname{sp}(L) \cap C(0,1)$ is finite and does not contain a point of the residual spectrum. Then $L^{n} x \rightarrow 0$ for every $x \in X$.

Proof. Denote the points of $\operatorname{sp}(L) \cap C(0,1)$ by $\lambda_{j}, j=1, \ldots, q$ and set

$$
p(L)=\left(L-\lambda_{1} I\right) \ldots\left(L-\lambda_{q} I\right) .
$$

This time $I-z L=-z(L-I / z)$ will be invertible at every point $z=\zeta \in C(0,1)$ different from the points $\zeta_{j}=1 / \lambda_{j}$. Thus the function $\sum_{0}^{\infty} L^{n} z^{n}$ has an analytic continuation to a neighborhood of such points $\zeta$. The same will be true for

$$
f(z)=\sum_{0}^{\infty} a_{n} z^{n} \stackrel{\text { def }}{=} \sum_{0}^{\infty} L^{n} p(L) z^{n}
$$

We will verify that for the singular points $\zeta_{j}$, the partial sums

$$
s_{n}\left(\zeta_{j}\right)=\sum_{0}^{n} L^{k} p(L) \zeta_{j}^{k}
$$

form a bounded sequence. Indeed, by the hypothesis

$$
s_{n}\left(\zeta_{j}\right)=p(L) \frac{\left(\zeta_{j} L\right)^{n+1}-I}{\zeta_{j} L-I}=\lambda_{j} \prod_{m \neq j}\left(L-\lambda_{m} I\right) \cdot\left\{\left(\zeta_{j} L\right)^{n+1}-I\right\}
$$

is in norm bounded by $(\|L\|+1)^{q-1}(M+1)$.

Thus by an operator analog of Theorem 13.4 or Theorem 14.5 for the simple case of a finite singularity set $E$, one has $L^{n} p(L) x \rightarrow 0$ for every $x \in X$. Now the points $\lambda_{j}$ are not in the residual spectrum of $L$; hence the ranges of the operators $L-\lambda_{j} I$ are dense in $X$. It now follows by approximation that the elements $p(L) x$ are dense in $X$, so that also $L^{n} y \rightarrow 0$ for every $y \in X$.

The following extension of Proposition 15.3 to the case of countably infinite peripheral spectrum is more difficult.

Theorem 15.4. Let $L$ be a power bounded linear operator on $X$. Suppose that $\operatorname{sp}(L)$ meets $C(0,1)$ only in a countable set and that $C(0,1)$ contains no point of the residual spectrum of $L$ (no eigenvalue of $L$ if $X$ is reflexive). Then

$$
L^{n} x \rightarrow 0 \text { as } n \rightarrow \infty, \forall x \in X .
$$

Remarks 15.5. There is a more limited predecessor for operators on a Hilbert space due to Sz.-Nagy and Foiass 1970. Theorem 15.4 has a companion for continuous semigroups of operators. Let $\{L(t), t \geq 0\}$ be such a semigroup with generator $A$. If $\sup _{t \geq 0}\|L(t)\|<\infty, L(t) x$ is continuous for every $x \in X, \operatorname{sp}(A) \cap i \mathbb{R}$ is countable and the residual spectrum of $A$ does not meet $i \mathbb{R}$, then $L(t) x \rightarrow 0$ as $t \rightarrow \infty$ for every $x \in X$. Arendt and Batty [1988] obtained the above results by a method, based on a precise estimate in the proof of Theorem 13.4 by Allan, O'Farrell and Ransford [1987. Another proof is contained in the work of Lyubich and Vu 1988. A third proof was given by Esterle, Strouse and Zouakia [1990]. For more results on semigroups of operators, see Batty [1994b] and the book by Arendt, Batty, Hieber and Neubrander [2001]. 


\section{Complex REMAINDER THEORY}

There is an extensive body of Tauberian remainder theory in which the relevant transforms are subject to conditions in the complex domain; cf. parts of the books by Ganelius [1971] and Postnikov 1979], and especially the comprehensive treatment by Subhankulov [1976 Russian]. In this article we restrict ourselves to some striking examples of the strong results provided by complex remainder theory.

We begin with a remainder theorem associated with Fatou's theorem. The result was originally stated for Laplace integrals and derived by real approximation (Korevaar 1954b]). The proof in Section 17 below is more direct.

In Section 18 we use a method of Postnikov and Subhankulov to derive a complex remainder theorem for power series which corresponds to the Hardy-Littlewood theorem mentioned in Section 1. More general remainder theorems for Dirichlet series and Laplace integrals can be found in the book by Subhankulov.

Finally, in Section 19, we discuss a complex remainder estimate for the Stieltjes transform, due to Malliavin 1962 and Pleijel 1963. This result has been used to estimate the counting function for the eigenvalues in certain elliptic problems; cf. Agmon and Kannai [1967.

For the case of power series we will use

Proposition 16.1. Let $p \in \mathbb{N}$ be fixed and let $0<r<1$. Then for $\lambda$ running over $(0,1]$ and for $m$ running over $\mathbb{Z}$,

$$
\begin{gathered}
I_{m}(\lambda)=I_{m}(\lambda, p, r)=\int_{-\lambda}^{\lambda} \frac{\left(e^{i t}-e^{i \lambda}\right)^{p}\left(e^{i t}-e^{-i \lambda}\right)^{p}}{1-r e^{i t}} e^{i m t} d t \\
(16.1)= \begin{cases}\mathcal{O}\left(\frac{\lambda^{2 p}}{\lambda^{p}|m|^{p}+1}\right) & \text { for } m \geq-(2 p-1), \\
2 \pi r^{-2 p-m}\left(1-2 r \cos \lambda+r^{2}\right)^{p}+\mathcal{O}\left(\frac{\lambda^{2 p}}{\lambda^{p}|m|^{p}+1}\right) & \text { for } m \leq-2 p .\end{cases}
\end{gathered}
$$

Here the constants in the $\mathcal{O}$-terms may be taken independent of $r$ when $1-r \leq \lambda / 4$.

In Section 17 we need only the case of fixed $\lambda$ and error term $\mathcal{O}\left(\left\{1 /\left(|m|^{p}+1\right)\right\}\right)$. For this case the result is due to Postnikov, who used real analysis [1953, for $p=2$ ], 1979, for general $p$ ]. The more precise result which allows $\lambda \rightarrow 0$ is required in Section 18. It is contained in work of Subhankulov [1960, who used a complex method.

Proof of Proposition 16.1. Setting $e^{i t}=z$ one has

$$
I_{m}(\lambda)=\frac{1}{i} \int_{\Gamma} \frac{\left(z-e^{i \lambda}\right)^{p}\left(z-e^{-i \lambda}\right)^{p}}{1-r z} z^{m-1} d z
$$

where $\Gamma$ is the arc of the (positively oriented) unit circle from $e^{-i \lambda}$ to $e^{i \lambda}$. To estimate the integral we will change the path of integration.

For $m \geq 1$ we replace $\Gamma$ by the arc $\Gamma_{1}$ of the circle $|z-1|=\left|e^{i \lambda}-1\right|$ from $e^{-i \lambda}$ to $e^{i \lambda}$ on which $|z| \leq 1$. Since $|1-r z| \geq c \lambda$ on $\Gamma_{1}$, straightforward estimation shows that $I_{m}(\lambda)=\mathcal{O}\left(\lambda^{2 p}\right)$. Starting with $z^{m-1} d z=d z^{m} / m$ one can also integrate by parts $p$ times on $\Gamma_{1}$. This results in the estimate $I_{m}(\lambda)=\mathcal{O}\left(\lambda^{p} / m^{p}\right)$. It is convenient to combine the two estimates into the $\mathcal{O}$-term of the proposition.

Next suppose that $m \leq-2 p$. Assuming now that $1-r \leq \lambda / 4$ we replace the path of integration $\Gamma$ by the arc $\Gamma_{2}$ of the circle $|z-1|=\left|e^{i \lambda}-1\right|$ from $e^{-i \lambda}$ to $e^{i \lambda}$ 
on which $|z| \geq 1$. In doing so we pick up a residue at the pole of the integrand:

$$
\begin{aligned}
\int_{\Gamma}-\int_{\Gamma_{2}} & =-2 \pi i \times \text { residue of the integrand at } z=1 / r \\
& =2 \pi i\left(1-r e^{i \lambda}\right)^{p}\left(1-r e^{-i \lambda}\right)^{p} r^{-2 p-m} .
\end{aligned}
$$

Divided by $i$, this result gives the principal term in the second line of (16.1). To estimate the remainder from $\int_{\Gamma_{2}}$ one may proceed as in the case of $\Gamma_{1}$.

For $-2 p<m<1$ direct estimation on $\Gamma_{1}$ gives $I_{m}(\lambda)=\mathcal{O}\left(\lambda^{2 p}\right)$, which implies the desired estimate for these restricted $m$.

Corollary 16.2. Let $f(z)=\sum_{0}^{\infty} a_{k} z^{k}$ for $r=|z|<1$. Then for $\lambda \in(0,1]$, $1-r \leq \lambda / 4$ and $n \geq 2 p$,

$$
\begin{aligned}
& \int_{-\lambda}^{\lambda} f\left(r e^{i t}\right) \frac{\left(e^{i t}-e^{i \lambda}\right)^{p}\left(e^{i t}-e^{-i \lambda}\right)^{p}}{1-r e^{i t}} e^{-i n t} d t=\sum_{k=0}^{\infty} a_{k} r^{k} I_{k-n}(\lambda, p, r) \\
& =2 \pi r^{n-2 p}\left(1-2 r \cos \lambda+r^{2}\right)^{p} \sum_{k=0}^{n-2 p} a_{k}+\mathcal{O}\left(\sum_{k=0}^{\infty} \frac{\left|a_{k}\right| r^{k} \lambda^{2 p}}{\lambda^{p}|k-n|^{p}+1}\right) .
\end{aligned}
$$

For the proof one applies the proposition to each term in the series $f\left(r e^{i t}\right)=$ $\sum_{0}^{\infty} a_{k} r^{k} e^{i k t}$, taking $m=k-n$.

\section{Remainder in Fatou's theorem}

The comparison functions below involve slowly varying functions in the sense of Karamata [1930b], [1933]: positive (measurable) functions $L$ on $[0, \infty)$ such that $L(\rho t) / L(t) \rightarrow 1$ as $t \rightarrow \infty$ for every $\rho>0$. Such functions have a simple integral representation:

$$
L(t)=c(t) \exp \left\{\int_{0}^{t} \varepsilon(v) \frac{d v}{v}\right\}
$$

where $c(\cdot)>0$ (is measurable and) tends to a limit $c>0$ at $\infty$, while $\varepsilon(\cdot)$ is integrable over every finite interval $(0, a)$ and tends to 0 at $\infty$. Cf. Korevaar, van Aardenne-Ehrenfest and de Bruijn [1949], Seneta [1976], Bingham, Goldie and Teugels [1987.

Here we need $L$ only on the nonnegative integers and we may therefore assume that $L$ and hence $c(\cdot)$ is continuous. Then for any $\delta>0$, there are positive constants $b$ and $B$ such that

$$
b\left(\frac{u+1}{t+1}\right)^{-\delta} \leq \frac{L(u)}{L(t)} \leq B\left(\frac{u+1}{t+1}\right)^{\delta} \quad \text { whenever } 0 \leq t \leq u<\infty .
$$

Theorem 17.1. Let $\sum_{0}^{\infty} a_{n} z^{n}$ converge for $|z|<1$ and let the sum function $f(z)$ be analytic at the point $z=1$. Suppose that the $a_{n}$ are real and that

$$
a_{n} \geq-\phi(n)=-(n+1)^{\alpha} L(n), \quad \forall n,
$$

where $\alpha$ is real and $L(\cdot)$ is slowly varying. Then there is a constant $C$ (depending on $f$ and $\phi)$ such that for $s_{n}=\sum_{0}^{n} a_{k}$,

$$
\left|s_{n}-f(1)\right| \leq C \phi(n), \quad \forall n .
$$


The result is contained in Korevaar 1954b theorem 4.1]. The present proof extends a method of Heilbronn and Landau 1933b for the case $\phi(n)=c$ and of Postnikov [1979] for the case $\phi(n)=c(n+1)^{\alpha}$ with $\alpha>-1$. It will use Proposition 16.1 and the auxiliary results below. The proof also uses the fact that

$$
\phi(n)=\mathcal{O}\left\{(n+1)^{\alpha+\delta}\right\}, \quad(n+1)^{\alpha-\delta}=\mathcal{O}\{\phi(n)\}
$$

for every $\delta>0$; cf. (17.2).

Lemma 17.2. For $p \in \mathbb{N}$ and $\lambda>0$, let

$$
H_{p}(x)=\frac{1}{2 \pi}\left(\frac{\sin x / p}{x / p}\right)^{p}, \quad H_{p, \lambda}(x)=\lambda H_{p}(\lambda x) .
$$

Then the Fourier transform $\hat{H}_{p, \lambda}$ has support in $[-\lambda, \lambda]$ and is of class $B^{p-1}$ (indefinite integral of order $p-1$ of a bounded function). If $p \geq 2$, the derivatives of $\hat{H}_{p, \lambda}$ of order $\leq p-2$ will vanish at $\pm \lambda$.

Cf. the Fourier pair (4.7) which corresponds to the case $p=2$. For the proof one may change scale and consider the $p$-th power $M_{p}(x)$ of the simple kernel $M(x)=(\sin x) /(\pi x)$. The latter has Fourier transform $\hat{M}(t)$ equal to 1 for $|t|<1$ and equal to 0 for $|t|>1$. Thus $\hat{M}_{p}$ is the $p$-th convolution power of $\hat{M}$. Hence it has support $[-p, p]$ and is of class $B^{p-1}$. In particular its derivatives of order $\leq p-2$ vanish at $\pm p$. The lemma readily follows.

Observe that by Fourier inversion,

$$
\int_{-\lambda}^{\lambda} \hat{H}_{p, \lambda}(t) e^{i x t} d t=2 \pi H_{p, \lambda}(x)=\lambda\left(\frac{\sin \lambda x / p}{\lambda x / p}\right)^{p} .
$$

Proposition 17.3. Under the hypotheses of Theorem 17.1 there is a constant $C_{1}$ (depending on $f$ and $\phi$ ) such that

$$
\left|a_{n}\right| \leq C_{1} \phi(n), \quad \forall n .
$$

Proof. For $f\left(r e^{i t}\right)=\sum_{0}^{\infty} a_{k} r^{k} e^{i k t}, 0 \leq r<1$ and $n \geq 0$, formula (17.7) gives

$$
\begin{aligned}
& \int_{-\lambda}^{\lambda} \hat{H}_{p, \lambda}(t) f\left(r e^{i t}\right) e^{-i n t} d t=\sum_{k=0}^{\infty} a_{k} r^{k} \lambda\left(\frac{\sin \lambda(k-n) / p}{\lambda(k-n) / p}\right)^{p} \\
& =\lambda a_{n} r^{n}+\lambda^{1-p} p^{p} \sum_{k \geq 0, k \neq n} a_{k} r^{k} \frac{\sin ^{p} \lambda(k-n) / p}{(k-n)^{p}} .
\end{aligned}
$$

We continue with even $p=2 q$ and observe that the integral is real; hence (17.3) and (17.9) imply the inequality

$$
\lambda a_{n} r^{n} \leq \int_{-\lambda}^{\lambda} \hat{H}_{2 q, \lambda}(t) f\left(r e^{i t}\right) e^{-i n t} d t+\lambda^{1-2 q}(2 q)^{2 q} \sum_{k \geq 0, k \neq n} \frac{\phi(k)}{(k-n)^{2 q}} r^{k}
$$

Now fix $\lambda>0$ so small that $f$ is analytic on the $\operatorname{arc}\{|z|=1,|\arg z| \leq \lambda\}$ and take $2 q>|\alpha|+1$. In view of (17.5) (with $k$ instead of $n$ ) we may then pass to the limit as $r \nearrow 1$ and henceforth consider (17.10) with $r=1$.

To prove (17.8) one may take $n \geq 1$, write $e^{-i n t} d t=-d e^{-i n t} /(i n)$ and integrate by parts $2 q-1$ times. This will show that the integral with $r=1$ is $\mathcal{O}\left(n^{-2 q+1}\right)=$ $\mathcal{O}\left\{n^{\alpha} L(n)\right\}=\mathcal{O}\{\phi(n)\}$. (The integrated terms drop out.) To verify that the sum with $r=1$ is also $\mathcal{O}\{\phi(n)\}$ one first estimates the numbers $\phi(k)$ in terms of $\phi(n)$ with the aid of (17.2). The parts of the resulting sum where $k>2 n$ or $k<\frac{1}{2} n$ may 
be compared with integrals; the part where $\frac{1}{2} n \leq k \leq 2 n$ is easy. The conclusion is that indeed $a_{n}=\mathcal{O}\{(\phi(n)\}$.

Proof of Theorem 17.1. By changing the constant term in the power series for $f$ we may assume that $f(1)=0$. Observe that by Proposition 17.3 and (17.5) one has $a_{k}=\mathcal{O}\{\phi(k)\}=\mathcal{O}\left\{(k+1)^{\alpha+\delta}\right\}$ for every $\delta>0$. We now apply Corollary 16.2. Here we fix $\lambda \in(0,1]$ so small that $f(z)$, hence also $f(z) /(1-z)$, is analytic on the $\operatorname{arc}\{|z|=1,|\arg z| \leq \lambda\}$ and we take $p>|\alpha|+1$. Under our hypotheses we may then let $r \nearrow 1$.

From here on we use formula (16.3) with $r=1$ : for $n \geq 2 p$,

$$
\begin{aligned}
& \int_{-\lambda}^{\lambda} \frac{f\left(e^{i t}\right)}{1-e^{i t}}\left(e^{i t}-e^{i \lambda}\right)^{p}\left(e^{i t}-e^{-i \lambda}\right)^{p} e^{-i n t} d t \\
& =2 \pi(2-2 \cos \lambda)^{p} s_{n-2 p}+\mathcal{O}\left(\sum_{k=0}^{\infty} \frac{\phi(k)}{|k-n|^{p}+1}\right) .
\end{aligned}
$$

By the analyticity of $f(z) /(1-z)$ we may integrate by parts $p$ times in the integral to show that it is $\mathcal{O}\left(n^{-p}\right)=\mathcal{O}\{\phi(n)\}$. By the argument used in the proof of Proposition 17.3, the sum is likewise $\mathcal{O}\{\phi(n)\}$. It follows that $s_{n-2 p}=\mathcal{O}\{\phi(n)\}$ and this completes the proof of (17.4).

Remarks 17.4. The proof does not fully use analyticity of $f$ at the point $z=1$, but one needs appropriate smoothness in a neighborhood of 1 in the closed disc $\{|z| \leq 1\}$, depending on $\alpha$. Cf. also Kră̌nova [1984.

Further inequalities (Korevaar 1954b) may be derived by applying Theorem 17.1 to, for example,

$$
\sum_{0}^{\infty}\left\{s_{n}-f(1)\right\} z^{n}=-\frac{f(z)-f(1)}{z-1} .
$$

Since $\left|s_{n}-f(1)\right| \leq C \phi(n)$ we can use Theorem 17.1 with $a_{n}$ replaced by $s_{n}-f(1)$. The result is

$$
\left|\frac{s_{0}+\cdots+s_{n}}{n+1}-f(1)+\frac{f^{\prime}(1)}{n+1}\right| \leq C^{\prime} \frac{\phi(n)}{n+1} .
$$

\section{REMAINDERS IN HARDY-LitTLEWOOD THEOREMS FOR POWER SERIES}

Let the power series

$$
f(x)=\sum_{0}^{\infty} a_{n} x^{n}
$$

converge for $|x|<1$ and let $a_{n} \geq-C$. Assuming that

$$
(1-x) f(x) \rightarrow A \text { as } x \nearrow 1,
$$

Hardy and Littlewood [1914 proved that $s_{n}=\sum_{0}^{n} a_{k} \sim A n$ as $n \rightarrow \infty$. (A simple proof was given by Karamata [1930a].) If

$$
f(x)-\frac{A}{1-x}=\mathcal{O}\left(\frac{1}{(1-x)^{\alpha}}\right) \quad \text { for } 0 \leq x<1
$$

where $\alpha<1$, the remainder theory of Freud 1951-4] and Korevaar 1953], 1954a], 1954b. gave $s_{n}-A n=\mathcal{O}(n / \log n)$. Under the real condition (18.2), the 'disappointingly large' remainder $\mathcal{O}(n / \log n)$ is optimal. In fact, real conditions give 
smaller remainders $s_{n}-A n$ only if the remainder in (18.2) is very small. For example, a remainder $\mathcal{O}(\exp \{-c /(1-x)\})$ in (18.2) gives (optimal) remainder $s_{n}-A n=\mathcal{O}\left(n^{\frac{1}{2}}\right)$; cf. Korevaar [1954a].

In this section we treat the following complex remainder theorem which is contained in work of Subhankulov [1960] for Dirichlet series; see also his book [1976], chapter 2].

Theorem 18.1. Let the power series

$$
f(z)=\sum_{0}^{\infty} a_{n} z^{n}, \quad z=r e^{i t}
$$

with $a_{n} \geq-C$, converge for $|z|<1$. Let $0 \leq \alpha, \beta<1, c>0$ and suppose that

$$
f(z)-\frac{A}{1-z}=\mathcal{O}\left(\frac{1}{|1-z|^{\alpha}}\right) \quad \text { for } 0 \leq r<1,|t| \leq c(1-r)^{\beta}
$$

(which describes a region tangent to the unit circle at the point $z=1$ ). Then the partial sums $s_{n}$ satisfy

$$
s_{n}-A n=\mathcal{O}\left(n^{\alpha}\right)+\mathcal{O}\left(n^{\beta}\right) \text { as } n \rightarrow \infty .
$$

Here $n^{\alpha}$ has to be replaced by $\log n$ if $\alpha=0$.

Proof (first part). Subtracting $A$ from the numbers $a_{n}$ it may henceforth be assumed that $A=0$. One then has to prove that $s_{n}=\mathcal{O}\left(n^{\alpha}\right)+\mathcal{O}\left(n^{\beta}\right)$ or $\mathcal{O}(\log n)$ for $n \geq n_{0}$. To this end we will apply Corollary 16.2 with $p=2$ and $\lambda=c(1-r)^{\beta}$; it may of course be assumed that $\lambda \leq 1$ and $1-r \leq \lambda / 4$. The result is that for $n \geq 4$,

$$
\begin{aligned}
& \int_{-\lambda}^{\lambda} f\left(r e^{i t}\right) \frac{\left(e^{i t}-e^{i \lambda}\right)^{2}\left(e^{i t}-e^{-i \lambda}\right)^{2}}{1-r e^{i t}} e^{-i n t} d t \\
& =2 \pi r^{n-4}\left(1-2 r \cos \lambda+r^{2}\right)^{2} s_{n-4}+\lambda^{4} \mathcal{O}\left(\sum_{k=0}^{\infty} \frac{\left|a_{k}\right| r^{k}}{\lambda^{2}(k-n)^{2}+1}\right) .
\end{aligned}
$$

Here we take $1-r=1 / n$ so that the coefficient of $s_{n-4}$ is $\geq$ a positive constant times $\lambda^{4}$. By (18.4) with $A=0$, the integral in (18.6) is

$$
\mathcal{O}\left(\lambda^{4} \int_{-\lambda}^{\lambda} \frac{d t}{\left|1-r e^{i t}\right|^{\alpha+1}}\right)=\lambda^{4} \mathcal{O}\left(\int_{0}^{1} \frac{d t}{(1-r+t)^{\alpha+1}}\right)=\lambda^{4} \mathcal{O}\left\{(1-r)^{-\alpha}\right\}
$$

for $\alpha>0$ and $=\lambda^{4} \mathcal{O}(\log \{1 /(1-r)\})$ if $\alpha=0$. For our $r$ the result is $\lambda^{4} \mathcal{O}\left(n^{\alpha}\right)$ if $\alpha>0$ and $\lambda^{4} \mathcal{O}(\log n)$ if $\alpha=0$. We now complete the proof for

The case $\left|a_{k}\right| \leq C$. To estimate the sum in (18.6) we split it. The sum over the terms with $|k-n| \leq 1 / \lambda$ is obviously $\mathcal{O}(1 / \lambda)$, which for our $\lambda$ and $r$ becomes $\mathcal{O}\left(n^{\beta}\right)$. Likewise, the sum with $|k-n|>1 / \lambda$ is

$$
\mathcal{O}\left(\frac{1}{\lambda^{2}} \sum_{|k-n|>1 / \lambda} \frac{1}{(k-n)^{2}}\right)=\mathcal{O}(1 / \lambda)=\mathcal{O}\left(n^{\beta}\right) .
$$

This is also true if $\beta=0$. Inserting the estimates for integral and sum into (18.6) and dividing by $\lambda^{4}$, one obtains the desired result for the partial sums $s_{n-4}$ when $\left|a_{k}\right| \leq C$. 
For the general case of Theorem 18.1, we show first that under the one-sided condition $a_{k} \geq-C$, where we take $C>0$, certain running averages of the numbers $\left|a_{k}\right|$ are bounded.

Lemma 18.2. Let $f$ satisfy the conditions in Theorem 18.1 and suppose that $0<$ $\lambda=c(1-r)^{\beta} \leq 1,1-r=1 / n \leq \lambda / 4$ as in the proof above. Then

$$
\sum_{|k-m| \leq 1 / \lambda}\left|a_{k}\right| \leq \sum_{|k-m| \leq 1 / \lambda}\left(a_{k}+2 C\right)=\mathcal{O}(1 / \lambda)=\mathcal{O}\left(n^{\beta}\right),
$$

uniformly for $m$ satisfying $|m-n| \leq \sqrt{n / \lambda}$.

Proof. By formula (17.9) with $p=2$ and $m$ instead of $n$, cf. (4.7),

$$
\begin{aligned}
& \sum_{k=0}^{\infty}\left(a_{k}+2 C\right) r^{k}\left(\frac{\sin \lambda(k-m) / 2}{\lambda(k-m) / 2}\right)^{2} \\
& =\frac{1}{\lambda} \int_{-\lambda}^{\lambda}\left(1-\frac{|t|}{\lambda}\right) f\left(r e^{i t}\right) e^{-i m t} d t+2 C \sum_{k=0}^{\infty} r^{k}\left(\frac{\sin \lambda(k-m) / 2}{\lambda(k-m) / 2}\right)^{2} .
\end{aligned}
$$

Since in the integral one has $f\left(r e^{i t}\right)=\mathcal{O}\left(1 /|t|^{\alpha}\right)$ with $\alpha<1$, the first term on the right will be $\mathcal{O}\left(1 / \lambda^{\alpha}\right)$. By the computation in the preceding proof the final sum is $\mathcal{O}(1 / \lambda)$. Now for $|k-m| \leq 1 / \lambda,|m-n| \leq \sqrt{n / \lambda}$ and $r=1-1 / n$,

$$
r^{k} \geq r^{n+\sqrt{n / \lambda}+1 / \lambda} \geq c_{1}>0, \quad\left(\frac{\sin \lambda(k-m) / 2}{\lambda(k-m) / 2}\right)^{2} \geq c_{2}>0 .
$$

Thus (18.8) implies (18.7).

Proof of Theorem 18.1 (second part, the case $a_{k} \geq-C$ ). To complete the proof we need a good estimate for the final sum in (18.6) under the one-sided condition. This time we split the sum in a different manner. First let $|k-n|>\sqrt{n / \lambda}$ :

$$
\begin{aligned}
& \sum_{|k-n|>\sqrt{n / \lambda}} \frac{\left|a_{k}\right| r^{k}}{\lambda^{2}(k-n)^{2}+1} \\
\leq & \frac{1}{\lambda n} \sum_{k=0}^{\infty}\left(a_{k}+2 C\right) r^{k}=\frac{1}{\lambda n}\left(f(r)+\frac{2 C}{1-r}\right)=\mathcal{O}(1 / \lambda) .
\end{aligned}
$$

By Lemma 18.2 the sum with $|k-n|<1 / \lambda$ is also $\mathcal{O}(1 / \lambda)$. It remains to consider the sum with $1 / \lambda \leq|k-n| \leq \sqrt{n / \lambda}$ which we majorize by a sum over parts in which $|k-m| \leq 1 / \lambda$ for suitable $m$ 's. Decreasing $c$ as necessary one may assume that $1 / \lambda=n^{\beta} / c$ is an integer. Now for $m_{s}=n+2 s / \lambda$ with $s=1,2, \ldots$ and $\left|k-m_{s}\right| \leq 1 / \lambda$, one has

$$
\frac{1}{\lambda^{2}(k-n)^{2}+1}<\frac{1}{(2 s-1)^{2}}
$$

Thus by Lemma 18.2

$$
\begin{aligned}
& \sum_{1 / \lambda \leq k-n \leq \sqrt{n / \lambda}} \frac{\left|a_{k}\right| r^{k}}{\lambda^{2}(k-n)^{2}+1} \leq \sum_{1 \leq s \leq \frac{1}{2} \sqrt{\lambda n}} \sum_{\left|k-m_{s}\right| \leq 1 / \lambda} \frac{\left|a_{k}\right|}{\lambda^{2}(k-n)^{2}+1} \\
& \leq \sum_{1 \leq s \leq \frac{1}{2} \sqrt{\lambda n}} \frac{1}{(2 s-1)^{2}} \sum_{\left|k-m_{s}\right| \leq 1 / \lambda}\left|a_{k}\right|=\mathcal{O}(1 / \lambda) .
\end{aligned}
$$


Also considering $m_{s}^{\prime}=n-2 s / \lambda$ one obtains the same estimate for the sum with $-\sqrt{n / \lambda} \leq k-n \leq-1 / \lambda$. Combining the estimates one finds that the final sum in (18.6) is $\mathcal{O}(1 / \lambda)=\mathcal{O}\left(n^{\beta}\right)$. This completes the proof of the theorem.

Remark 18.3. Postnikov [1953] had treated the important case $\alpha=\beta=0$ of Theorem 18.1 which does not require letting $\lambda$ go to 0 . He showed also 1979 that in this case, the remainder estimate $s_{n}-A n=\mathcal{O}(\log n)$ in (18.5) is essentially optimal. However, if $g(z)=f(z)-A /(1-z)$ in Theorem 18.1 is analytic at $z=1$, it follows from Theorem 17.1 applied to $g$ that $s_{n}-A n=\mathcal{O}(1)$.

Remarks 18.4. Subhankulov [1964 also obtained remainder estimates for Littlewood's theorem based on complex information. As in Postnikov's exposition [1979. section 19] we restrict ourselves here to Littlewood's original Tauberian condition

$$
\left|n a_{n}\right| \leq C \text {. }
$$

If $f(x)=\sum_{0}^{\infty} a_{n} x^{n}$ tends to $A$ as $x \rightarrow 1$, then $s_{n}=\sum_{0}^{n} a_{k} \rightarrow A$ (Littlewood [1911]). In the case $f(x)-A=\mathcal{O}\left\{(1-x)^{\alpha}\right\}$ for $0 \leq x<1$ with $\alpha>0$, Freud-Korevaar remainder theory (loc. cit.) gave the optimal estimate $s_{n}-A=\mathcal{O}(1 / \log n)$.

Setting $z=r e^{i t}$, we now impose the complex condition

$$
f(z)-A=\mathcal{O}\left(|1-z|^{\alpha}\right) \text { for } 0 \leq r<1,|t| \leq c(1-r)^{\beta},
$$

where $0<\alpha \leq 1,0<\beta<1$. Under condition (18.11) Subhankulov then found that

$$
s_{n}-A=\mathcal{O}\left(n^{\beta-1}\right)+\mathcal{O}\left(n^{-\alpha \beta}\right) .
$$

Observe that there must be a better result for small $\beta$. Indeed, take $\alpha=1$. Then the estimate $s_{n}-A=\mathcal{O}\left(n^{-\frac{1}{2}}\right)$ for $\beta=\frac{1}{2}$ must also hold for $\beta=0$; see (18.12). The question of an optimal result seems to be open. In his book, Subhankulov [1976, section 2.6] found, among other things, that condition (18.12) together with the corresponding condition on $(1-z) f^{\prime}(z)$ implies

$$
s_{n}-A=\mathcal{O}\left(n^{\beta-1}\right)+\mathcal{O}\left(n^{-\alpha}\right) .
$$

In all of this, (18.11) may be replaced by the one-sided condition $n a_{n} \geq-C$ of Hardy and Littlewood [1914], provided $\sum a_{n} z^{n}$ is known to converge for $|z|<1$.

\section{Remainder For the Stieltjes transform}

Let $s(0)=0$, and let $s(t)$ be nondecreasing for $t \geq 0$ and such that the Stieltjes transform

Suppose that

$$
g(x)=\int_{0}^{\infty} \frac{d s(t)}{t+x} \quad \text { exists for } x>0
$$

$$
g(x)=A x^{\alpha-1}+o\left(x^{\alpha-1}\right) \quad \text { as } x \rightarrow \infty,
$$

where $0<\alpha<1$ and $A \geq 0$. Then by Tauberian theory of Hardy and Littlewood 1929,

$$
s(t)=A^{\prime} t^{\alpha}+o\left(t^{\alpha}\right) \quad \text { as } t \rightarrow \infty, \quad \text { where } A^{\prime}=A(\sin \alpha \pi) /(\alpha \pi) .
$$

A more direct proof was given by Karamata [1931]. As in the case of power series, real remainder formulas for the transform give only weak remainder formulas for $s(t)$. In the real case one needs an extremely small remainder $g(x)-A x^{\alpha-1}$ in order 
to get a small remainder $s(t)-A^{\prime} t^{\alpha}$ (Avakumović [1950]; cf. Subhankulov [1976] chapter 3]).

Using complex asymptotics for the Stieltjes transform, Malliavin [1962] and Pleijel [1963] obtained the following useful remainder theorem.

Theorem 19.1. Let $s(\cdot), \alpha, A$ and $A^{\prime}$ be as above, $0<\beta<\alpha<1$ and $0 \leq \gamma<1$. Suppose that

$$
g(z)=\int_{0}^{\infty} \frac{d s(t)}{t+z}=A z^{\alpha-1}+\mathcal{O}\left(|z|^{\beta-1}\right)
$$

on the two curves $z=x \pm i|x|^{\gamma},-\infty<x \leq-1$. Here $z^{\alpha-1}$ denotes the principal value. Then

$$
s(t)-A^{\prime} t^{\alpha}=\mathcal{O}\left(t^{\beta}\right)+\mathcal{O}\left(t^{\alpha-1+\gamma}\right) \quad \text { as } t \rightarrow \infty .
$$

The proof below, after Pleijel, starts with an important approximate inversion formula for the Stieltjes transform.

Lemma 19.2. Let $w=u+i v$ with $u<0, v>0$ and let $L(w)$ be any rectifiable arc from $\bar{w}$ to $w$ which does not meet the negative real axis $\{y=0, x \leq 0\}$. Define

$$
I(w)=\frac{1}{2 \pi i} \int_{L(w)} g(z) d z
$$

and set $g=g_{1}+i g_{2}$ with real $g_{j}$. Then

$$
\left|s(|u|)-I(w)+\frac{1}{\pi} v g_{1}(w)\right| \leq \frac{1}{2} v\left|g_{2}(w)\right| .
$$

Proof. Let $\phi=\phi(t, w)$ be the angle between the positive real direction at the point $z=-t$ and the direction from $z=-t$ to $z=w$. Then by (19.1)

$$
\begin{aligned}
& v g_{1}(w)=\int_{0}^{\infty} \frac{(t+u) v}{(t+u)^{2}+v^{2}} d s(t)=\int_{0}^{\infty} \cos \phi \sin \phi d s(t), \\
& v g_{2}(w)=\int_{0}^{\infty} \frac{-v^{2}}{(t+u)^{2}+v^{2}} d s(t)=-\int_{0}^{\infty} \sin ^{2} \phi d s(t) .
\end{aligned}
$$

Introducing the definition of $g$ into (19.3) and inverting the order of integration, one obtains

$$
I(w)=\frac{1}{2 \pi i} \int_{0}^{\infty} d s(t) \int_{L(w)} \frac{d z}{t+z}=\frac{1}{\pi} \int_{0}^{\infty} \phi(t, w) d s(t) .
$$

Hence by (19.5)

$$
\begin{aligned}
& s(|u|)-I(w)+\frac{1}{\pi} v g_{1}(w) \\
& =\frac{1}{\pi} \int_{0}^{|u|}\left(\pi-\phi+\frac{1}{2} \sin 2 \phi\right) d s(t)+\frac{1}{\pi} \int_{|u|}^{\infty}\left(-\phi+\frac{1}{2} \sin 2 \phi\right) d s(t) .
\end{aligned}
$$

A drawing helps to verify that $0 \leq t \leq|u|$ implies $\frac{1}{2} \pi \leq \phi \leq \pi$, while $t \geq|u|$ implies $0 \leq \phi \leq \frac{1}{2} \pi$. In the first case one has $\left|\pi-\phi+\frac{1}{2} \sin 2 \phi\right| \leq \frac{1}{2} \pi \sin ^{2} \phi$, while in the second case $\left|-\phi+\frac{1}{2} \sin 2 \phi\right| \leq \frac{1}{2} \pi \sin ^{2} \phi$. Thus (19.4) follows from (19.7) and (19.5). 
Proof of Theorem 19.1. Let $L$ be a positively oriented curve which consists of the arcs $y= \pm|x|^{\gamma}$ with $x \leq-1$ and a connecting rectifiable arc in the half-plane $\{x \geq-1\}$ which does not meet the half-line $\{y=0, x \leq 0\}$. Taking $w=u+i v$ on $L$ with $u<-1$ and $v>0$ we let $L(w)$ be the part of $L$ from $\bar{w}$ to $w$. We split $I(w)$ as follows:

$$
I(w)=\frac{1}{2 \pi i} \int_{L(w)}\left\{g(z)-A z^{\alpha-1}\right\} d z+\frac{A}{2 \pi i} \int_{L(w)} z^{\alpha-1} d z .
$$

By (19.1) the first term on the right is $\mathcal{O}\left(|w|^{\beta}\right)=\mathcal{O}\left(|u|^{\beta}\right)$ as $w \rightarrow \infty$ along $L$. The final term equals

$$
\frac{A}{2 \pi i} \frac{w^{\alpha}-(\bar{w})^{\alpha}}{\alpha}=\frac{A}{\alpha \pi}|w|^{\alpha} \sin \alpha \psi, \quad \psi=\arg w .
$$

Now for $w \rightarrow \infty$ along $L$,

$$
\begin{aligned}
|w|^{\alpha} & =|u|^{\alpha}+\mathcal{O}\left(|u|^{\alpha-1} v\right)=|u|^{\alpha}+\mathcal{O}\left(|u|^{\alpha-1+\gamma}\right), \\
\sin \alpha \psi & =\sin \{\alpha \pi-\alpha(\pi-\psi)\}=\sin \alpha \pi+\mathcal{O}(|v| /|w|)=\sin \alpha \pi+\mathcal{O}\left(|u|^{\gamma-1}\right) .
\end{aligned}
$$

Putting it all together we obtain from (19.8) that

$$
I(w)=\mathcal{O}\left(|u|^{\beta}\right)+\frac{A \sin \alpha \pi}{\alpha \pi}|u|^{\alpha}+\mathcal{O}\left(|u|^{\alpha-1+\gamma}\right) .
$$

Combination with (19.4) gives (19.2).

Remarks 19.3. Theorem 19.1 was extended to other Stieltjes-type transforms and considerably generalized by Subhankulov et al. See Subhankulov and An [1974, Subhankulov [1976, chapter 4]. A different generalization was obtained by Ganelius 1964, [1971, chapter 7].

Applications 19.4. Beginning with Valiron 1914 and Titchmarsh [1927, methods of Tauberian character for Stieltjes-type transforms have been used extensively to study the counting function for the zeros of entire functions. The simplest case is that of an entire function $f$ of order $\rho \in(0,1)$ whose zeros are negative real. If $f(0)=1$, then for the branch of the logarithm which is real on $\mathbb{R}^{+}$,

$$
\log f(z)=\int_{0}^{\infty} \log \left(1+\frac{z}{t}\right) d n(t)=z \int_{0}^{\infty} \frac{n(t)}{t(t+z)} d t,
$$

where $n(t)$ is the number of zeros of $f$ of absolute value $\leq t$. Thus growth estimates for $f$ on suitable curves give estimates for a Stieltjes-type transform which can be used to obtain results for $n(t)$. See the books by Boas 1954, Levin 1964, Bingham, Goldie and Teugels [1987] chapter 7] for asymptotic results, and the book by Subhankulov [1976] for remainder theorems.

Applications to eigenvalues go back to Carleman [1934]; cf. also Titchmarsh [1958, chapters 17, 22] and Agmon [1965, section 14]. Remainder theorems have been used for precise results on the number $N(t)$ of eigenvalues $\lambda_{j}$ with absolute value $\leq t$ in elliptic boundary value problems. Under appropriate conditions one may use resolvents or spectral densities to derive a formula of the type

$$
\sum_{j} \frac{1}{\lambda_{j}^{2 k}+z}=\int_{0}^{\infty} \frac{d N\left(t^{1 /(2 k)}\right)}{t+z}=A z^{q /(2 k)-1}+\mathcal{O}\left(z^{q /(2 k)-1-\delta}\right), \quad \delta>0,
$$


valid on curves as in Theorem 19.1. The theorem then gives an estimate for $N\left(t^{1 /(2 k)}\right)$ which leads to a conclusion of the form

$$
N(t)=A^{\prime} t^{q}+\mathcal{O}\left(t^{q-\eta}\right) \quad \text { with } \eta>0 .
$$

See for example Agmon and Kannai [1967. For a survey of developments and later results on eigenvalues in elliptic problems, see Hörmander [1983/5 chapters 17, 29].

\section{A FEW OPEN QUESTIONS}

Question 20.1. Newman's contour integration method (Sections 6, 7) provides a beautiful proof for the prime number theorem. Nevertheless the Wiener-Ikehara way to the PNT (Sections 4, 2) is somewhat more direct. Is it possible to derive the Wiener-Ikehara theorem by simple complex analysis? (The author proposed this question to Newman at a small analysis conference of Ganelius in Gothenburg, 1976.)

Question 20.2. Best $L^{1}$ approximation by Fourier transforms of functions with bounded support plays a central role in the Tauberian work of Graham and Vaaler (Section 5). Related approximation arose in Problem 11.1. It would be interesting to know if the extremal kernel described in Proposition 11.2 is unique; cf. Ingham [1936].

Question 20.3. Propositions 13.3 and 14.4 provide extensions of Fatou's theorem for the case where $f$ has just one singular point on the circle $C(0,1)$, here taken as the point $z=1$. Can the condition $\left|s_{n}\right| \leq C$ be weakened?

Question 20.4. In Theorems 13.4, 13.5 and 14.5 it is required that certain boundary singularity sets have linear measure zero. Can this condition be relaxed?

Question 20.5. Unlike the Hardy-Littlewood case in Theorem 18.1, there seems to be no satisfactory complex remainder result corresponding to Littlewood's theorem; cf. Remarks 18.4. One might study this discrepancy.

Added in proof. The extremal kernel in Proposition 11.2, which is the subject of Question 20.2, is indeed unique. This may be derived from a report on a related extremal problem by B. I. Johansson, An extremal property for the $L^{1}(\mathbb{R})$ functions with compactly supported Fourier transform, preprint, Dept. of Mathematics, Chalmers University of Technology, Gothenburg, 1993.

\section{REFERENCES}

Abel, N. H.

[1826] Recherches sur la série $1+\frac{m}{1} x+\frac{m(m-1)}{1 \cdot 2} x^{2}+\frac{m(m-1)(m-2)}{1 \cdot \cdot 2 \cdot 3} x^{3}+\cdots$. J. für Math. 1, 311-339. Oeuvres complètes, vol. I, pp 219-250. [Sec 1]

Agmon, S.

[1953] Complex variable Tauberians. Trans. Amer. Math. Soc. 74, 444-481. [Sec 4] MR 14:869a

[1965] Lectures on elliptic boundary value problems. Van Nostrand, New York. [Sec 19] MR 31:2504

Agmon, S. and Kannai, P.

[1967] On the asymptotic behavior of spectral functions and resolvent kernels of elliptic operators. Israel J. Math. 5, 1-30. [Sec 16, 19] MR 36:1814] 
Allan, G. R., O'Farrell, A. G. and Ransford, T. J.

[1987] A Tauberian theorem arising in operator theory. Bull. London Math. Soc. 19, 537-545. [Sec 1, 13, 15] MR 89c:47003

Apostol, T. M.

[2000] A centennial history of the prime number theorem. Number theory, pp 1-14. Trends in Math., Birkhäuser, Basel. [Sec 1] MR 2001d:11092

Aramaki, J.

[1996] An extension of the Ikehara Tauberian theorem and its application. Acta Math. Hungar. 71, 297-326. [Sec 4] MR 97e:35125]

Arendt, W. and Batty, C. J. K.

[1988] Tauberian theorems and stability of one-parameter semigroups. Trans. Amer. Math. Soc. 306, 837-852. [Sec 1, 13, 15] MR 89g:47053

[1995] A complex Tauberian theorem and mean ergodic semigroups. Semigroup Forum 50, 351366. [Sec 13] MR 96i:47016

Arendt, W., Batty, C. J. K., Hieber, M. and Neubrander, F.

[2001] Vector-valued Laplace transforms and Cauchy problems. Birkhäuser, Basel. [Sec 1, 13, 15] CMP 2002:09

Arendt, W. and Prüss, J.

[1992] Vector-valued Tauberian theorems and asymptotic behavior of linear Volterra equations. SIAM J. Math. Anal. 23, 412-448. [Sec 13] MR 92m:47150

Avakumović, V. G.

[1950] Bemerkung über einen Satz des Herrn T. Carleman. Math. Z. 53, 53-58. [Sec 19] MR 12:254a

Bateman, P. T. and Diamond, H. G.

[1996] A hundred years of prime numbers. Amer. Math. Monthly 103, 729-741. [Sec 1] MR 97m:01036

Batty, C. J. K.

[1990] Tauberian theorems for the Laplace-Stieltjes transform. Trans. Amer. Math. Soc. 322, 783-804. [Sec 13] MR 91c:44001]

[1994a] Some Tauberian theorems related to operator theory. Functional analysis and operator theory (Warsaw 1992), Banach Center Publ. 30, Warsaw, pp 21-34. [Sec 13] MR 95f:44001

[1994b] Asymptotic behaviour of semigroups of operators. Functional analysis and operator theory (Warsaw 1992), Banach Center Publ. 30, Warsaw, pp 35-52. [Sec 15] MR 95g:47058

Batty, C. J. K., van Neerven, J. and Räbiger, F.

[1998] Tauberian theorems and stability of solutions of the Cauchy problem. Trans. Amer. Math. Soc. 350, 2087-2103. [Sec 13] MR 98h:47055

Binder, C.

[1984] Alfred Tauber (1866-1942). Ein österreichischer Mathematiker. Jahrb. Überblicke Math. 1984, Math. Surv. 17, 151-166. [Sec 1]

Bingham, N. H., Goldie, C. M. and Teugels, J. L.

[1987] Regular variation. Encyclopedia of Mathematics and its Applications, vol. 27, Cambridge Univ. Press, Cambridge. (Paperback edition with additions, 1989.) [Sec 1, 17, 19] MR 90i:26003

Boas, R. P.

[1954] Entire functions. Academic Press, New York. [Sec 19]

Bochner, S.

[1933] Ein Satz von Landau und Ikehara. Math. Z. 37, 1-9. [Sec 4, 6]

Carleman, T.

[1934] Propriétés asymptotiques des fonctions fondamentales des membranes vibrantes. C. R. du $8^{e}$ Congrès des Math. Scand., Stockholm, pp 34-44. [Sec 19]

Chandrasekharan, K.

[1968] Introduction to analytic number theory. Grundl. math. Wiss. vol. 148, Springer, Berlin. [Sec 4] MR 40:2593

Chill, R.

[1998] Tauberian theorems for vector-valued Fourier and Laplace transforms. Studia Math. 128, 55-69. [Sec 13] MR 98k:44001] 
Č́ižek, J.

[1999] On the Tauberian constant in the Ikehara theorem. Czechoslovak Math. J. 49 (124), 673-682. [Sec 4] MR 2001d:11091

Delange, $\mathrm{H}$.

[1952] Encore une nouvelle démonstration du théorème taubérien de Littlewood. Bull. Sci. Math. (2) 76, 179-189. [Sec 1] MR 14:634g

[1954] Généralisation du théorème de Ikehara. Ann. Sci. École Norm. Sup. (3) 71, 213-242. [Sec 4] MR 16:921e

[1955] Théorèmes taubériens et applications arithmétiques. Mém. Soc. Roy. Liège (4) 16, 1-87. [Sec 4] MR 17:965c

[1997] Un théorème taubérien donnant des résultats équivalents au théorème des nombres premiers. Gaz. Math. No. 71, 39-58. [Sec 6] MR 98j:11068

Diamond, H. G.

[1972] On a Tauberian theorem of Wiener and Pitt. Proc. Amer. Math. Soc. 31, 152-158. [Sec 9] MR 45:4012

[1982] Elementary methods in the study of the distribution of prime numbers. Bull. Amer. Math. Soc. (N.S.) 7, 553-589. [Sec 1] MR 83m:10002

Doetsch, G.

[1937] Theorie und Anwendung der Laplace-Transformation. Grundl. math. Wiss. vol. 47, Springer, Berlin. [Sec 4] MR 5:119f

[1950] Handbuch der Laplace-Transformation, vol. I. Birkhäuser, Basel. [Sec 4] MR 13:230f

Duren, P. L.

[1970] Theory of $H^{p}$ spaces, Academic Press, New York. [Sec 13] MR 42:3552

Elliott, P. D. T. A.

[1979] Probabilistic number theory, vol. 1. Grundl. math. Wiss. vol. 239, Springer, Berlin. [Sec 4] MR 82h:10002a

Erdős, P.

[1949] On a new method in elementary number theory which leads to an elementary proof of the prime number theorem. Proc. Nat. Acad. Sci. U.S.A. 35, 374-384. [Sec 1] MR 10:595c

Esterle, J.

[1983] Quasimultipliers, representations of $H^{\infty}$, and the closed ideal problem for commutative Banach algebras. In: Radical Banach algebras and automatic continuity, Lecture Notes in Math. nr 975, Springer, Berlin, pp 66-162. [Sec 15] MR 85g:46067

Esterle, J., Strouse, E. and Zouakia, F.

[1990] Theorems of Katznelson-Tzafriri type for contractions. J. Funct. Anal. 94, 273-287. [Sec Fatou, P. 15] MR 92c: 47016

[1905] Sur quelques théorèmes de Riemann. Comptes rendus Acad. Sci. Paris 140, 569-570. [Sec 1]

[1906] Séries trigonométriques et séries de Taylor. Acta Math. 30, 335-400. [Sec 1, 12]

Freud, G.

[1951-4] Restglied eines Tauberschen Satzes. I, Acta Math. Acad. Sci. Hung. 2 (1951), 299-308; II, ibid. 3 (1953), 299-307; III, ibid. 5 (1954), 275-288. [Sec 1, 18] MR 14:361a MR 14:958a, MR 17:260c

Frobenius, G.

[1880] Über die Leibnitzsche Reihe. J. Reine Angew. Math. 89, 262-264. [Sec 1]

Gaier, D.

[1953] Complex Tauberian theorems for power series. Trans. Amer. Math. Soc. 75, 48-68. [Sec 12] MR 15:113c

Ganelius, T. H.

[1964] Tauberian theorems for the Stieltjes transform. Math. Scand. 14, 213-219. [Sec 19] MR 31:1496

[1971] Tauberian remainder theorems. Lect. Notes in Math. nr 232, Springer, Berlin. [Sec 1, 16, 19] MR 58:17647

Gel'fand, I. M.

[1941] Zur Theorie der Charaktere der abelschen topologischen Gruppen. Mat. Sbornik, N.S. 9 (51), 49-50. [Sec 15] MR 3:36d 
Graham, S. W. and Vaaler, J. D.

[1981] A class of extremal functions for the Fourier transform. Trans. Amer. Math. Soc. 265, 283-302. [Sec 1, 4, 5] MR 82i:42008

Hadamard, J.

[1896] Sur la distribution des zéros de la fonction $\zeta(s)$ et ses conséquences arithmétiques. Bull. Soc. Math. France (24), 199-220. [Sec 1]

Halász, G.

[1967] Remarks to a paper of D. Gaier on gap theorems. Acta Sci. Math. (Szeged) 28, 311-322. [Sec 1] MR 36:4199]

[1968] Über die Mittelwerte multiplikativer zahlentheoretischer Funktionen. Acta Math. Acad. Sci. Hungar. 19, 365-403. [Sec 4] MR 37:6254

Hardy, G. H.

[1949] Divergent Series. Clarendon Press, Oxford. [Sec 1] MR 11:25a

Hardy, G. H. and Littlewood, J. E.

[1914] Tauberian theorems concerning power series and Dirichlet's series whose coefficients are positive. Proc. London Math. Soc. (2) 13, 174-191. [Sec 1, 18]

[1918] Contributions to the theory of the Riemann zeta-function and the theory of the distribution of primes. Acta Math. 41, 119-196. [Sec 1]

[1929] Notes on the theory of series (XI): On Tauberian theorems. Proc. London Math. Soc. (2) 30, 23-37. [Sec 19]

Heilbronn, H. and Landau, E.

[1933a] Bemerkung zur vorstehenden Arbeit von Herrn Bochner. Math. Z. 37, 10-16. [Sec 4, 6]

[1933b] Ein Satz über Potenzreihen. Math. Z. 37, 17. [Sec 17]

[1933c] Anwendungen der N. Wienerschen Methode. Math. Z. 37, 18-21. [Sec 4, 6]

Heins, M.

[1968] Complex function theory. Academic Press, New York. [Sec 4] MR 39:413

Holt, J. J. and Vaaler, J. D.

[1996] The Beurling-Selberg extremal functions for a ball in Euclidean space. Duke Math. J. 83, 203-248. [Sec 5] MR 97f:30038

Hörmander, L.

[1983/5] The analysis of linear partial differential operators, vols 1-4. Grundl. math. Wiss. vols 256, 257, 274, 275, Springer, Berlin. [Sec 19] MR 85g:35002a MR 85g:35002b, corrected reprint MR 95h:35255 corrected reprint MR 98f:35002

Ikehara, S.

[1931] An extension of Landau's theorem in the analytic theory of numbers. J. Math. and Phys. M.I.T. 10, 1-12. [Sec 1, 2, 4]

Ingham, A. E.

[1932] The distribution of prime numbers. Cambridge Tracts in Math. no. 30, Cambridge Univ. Press. (Reprinted 1990.) [Sec 1, 2] MR 91f:11064

[1935] On Wiener's method in Tauberian theorems. Proc. London Math. Soc. (2) 38, 458-480. $[\operatorname{Sec} 1,4,6,7,9,10,12,13]$

[1936] Some trigonometrical inequalities with applications to the theory of series. Math. Z. 41, 367-379. [Sec 1, 10, 11, 12, 20]

[1941] A Tauberian theorem for partitions. Ann. of Math. (2) 42, 1075-1090. [Sec 4] MR 3:166a

[1950] A further note on trigonometrical inequalities. Proc. Cambridge Phil. Soc. 46, 535-537. [Sec 12] MR 12:255b

Jurkat, W. B.

[1956] Ein funktionentheoretischer Beweis für O-Taubersätze bei Potenzreihen. Arch. Math. 7, 122-125. [Sec 1] MR 18:31e]

[1957] Über die Umkehrung des Abelschen Stetigkeitssatzes mit funktionentheoretischen Methoden. Math. Z. 67, 211-222. [Sec 1] MR 19:544a

Karamata, J.

[1930a] Über die Hardy-Littlewoodschen Umkehrungen des Abelschen Stetigkeitssatzes. Math. Z. 32, 319-320. [Sec 18]

[1930b] Sur un mode de croissance régulière des fonctions. Mathematica (Cluj) 4, 38-53. [Sec 17 
[1931] Neuer Beweis und Verallgemeinerung der Tauberschen Sätze, welche die Laplacesche and Stieltjessche Transformation betreffen. J. reine u. angew. Math. 164, 27-39. [Sec $19]$

[1933] Sur un mode de croissance régulière. Théorèmes fondamentaux. Bull. Soc. Math. France 61, 55-62. [Sec 17]

[1934] Weiterführung der N. Wienerschen Methode. Math. Z. 38, 701-708. [Sec 7, 10]

[1936] Über einen Satz von Heilbronn und Landau. Publ. math. Univ. Belgrade 5, 28-38. [Sec $6]$

Katznelson, Y. and Tzafriri, L.

[1986] On power bounded operators. J. Funct. Anal. 68, 313-328. [Sec 1, 13, 14, 15] MR 88e: 47006

Korevaar, J.

[1951] An estimate of the error in Tauberian theorems for power series. Duke Math. J. 18, 723-734. [Sec 1] MR 13:227e]

[1953] Best $L^{1}$ approximation and the remainder in Littlewood's theorem. Indag. Math. 15, 281-293. [Sec 1, 18] MR 15:119c

[1954a] A very general form of Littlewood's theorem. Indag. Math. 16, 36-45. [Sec 1, 18] MR 15:698c

[1954b] Another numerical Tauberian theorem for power series. Indag. Math. 16, 46-56. [Sec 1, 12, 16, 17, 18] MR 15:698d

[1982] On Newman's quick way to the prime number theorem. Math. Intelligencer 4, 108-115. [Sec 1, 6, 7] MR 84b:10063

Korevaar, J., van Aardenne-Ehrenfest, T. and de Bruijn, N. G.

[1949] A note on slowly oscillating functions. Nieuw Arch. Wiskunde (2) 23, 77-86. [Sec 17] MR 10:358b

Kraĭnova, L. I.

[1984] A Tauberian theorem of Fatou type for power series in the form of explicit inequalities (Russian). Dokl. Akad. Nauk Tadzhik. SSR 27, 550-552. [Sec 17] MR 86e:30005]

Landau, E.

[1908] Zwei neue Herleitungen für die asymptotische Anzahl der Primzahlen unter einer gegegenen Grenze. Sitz. Ber. Preuss. Akad. Wiss. Berlin, 746-764. [Sec 1, 2]

[1909] Handbuch der Lehre von der Verteilung der Primzahlen vols I, II. Teubner, Leipzig. (Second edition with an appendix by P. T. Bateman, Chelsea Publ. Co., New York, 1953.) $[\operatorname{Sec} 1,2,8]$

[1912] Über einige neuere Grenzwertsätze. Rend. Palermo 34, 121-131. [Sec 6]

[1932a] Über den Wienerschen neuen Weg zum Primzahlsatz. Sitz.-Ber. Preuss. Akad. Wiss., Phys.-Math. Klasse, 1932, 514-521. [Sec 4, 6]

[1932b] Über Dirichletsche Reihen. Nachrichten Göttingen, 525-527. [Sec 4, 6]

Landau, E. and Gaier, D.

[1986] Darstellung und Begründung einiger neuerer Ergebnisse der Funktionentheorie, third enlarged edition. Springer, Berlin. (First and second edition 1916, 1929 by E. Landau.) [Sec 1, 2, 12] MR 88d:01046

Lang, S.

[1999] Complex analysis. Grad. Texts in Math., vol. 103, fourth edition. Springer, New York. [Sec 6] MR 99i:30001

Lavrik, A. F.

[1984] Methods of studying the law of distribution of primes (Russian). Trudy Mat. Inst. Steklov 163, 118-142. AMS Translation [1985] Proc. Steklov Inst. Math. 163, 141-167. [Sec 1] MR 86d:11070

Levin, B. Ja.

[1964] Distribution of zeros of entire functions. Transl. Math. Mono. 5, Amer. Math. Soc., Providence, RI. (Second edition 1980, original Russian edition 1956.) [Sec 19] MR 81k:30011

Levinson, N.

[1969] A motivated account of an elementary proof of the prime number theorem. Amer. Math. Monthly 76, 225-245. [Sec 1] MR 39:2712

Littlewood, J. E.

[1911] The converse of Abel's theorem on power series. Proc. London Math. Soc. (2) 9, 434-448. $[\operatorname{Sec} 1,18]$ 
[1971] The quickest proof of the prime number theorem. Acta Arith. 18, 83-86. [Sec 1] MR 45:3343

Lyubich, Yu. I. and Vu, Q. P.

[1988] Asymptotic stability of linear differential equations in Banach spaces. Studia Math. 88, 37-42. [Sec 15] MR 89e:47062

Malliavin, $\mathrm{P}$.

[1962] Un théorème taubérien avec reste pour la transformation de Stieltjes. C. R. Acad. Sci. Paris 255, 2351-2352. [Sec 1, 16, 19] MR 26:1662

Montgomery, H. L.

[1978] The analytic principle of the large sieve. Bull. Amer. Math. Soc. 84, 547-567. [Sec 5] MR 57:5931

[1994] Ten lectures on the interface between analytic number theory and harmonic analysis. CBMS Regional Conf. Series in Math. vol. 84, Amer. Math. Soc., Providence, RI. [Sec 5] MR 96i:11002

Narkiewicz, W.

[2000] The development of prime number theory. Springer Monographs in Math., Springer, Berlin. [Sec 1] MR 2001c:11098

Newman, D. J.

[1980] Simple analytic proof of the prime number theorem. Amer. Math. Monthly 87, 693-696. [Sec 1, 6, 7] MR 82h:10056

[1998] Analytic number theory. Grad. Texts in Math. vol. 177, Springer, New York. [Sec 6] MR 98m:11001

Paley, R. E. A. C. and Wiener, N.

[1934] Fourier transforms in the complex domain. Amer. Math. Soc. Colloq. Publ. vol. 19, Amer. Math. Soc., New York. [Sec 5]

Pitt, H. R.

[1958] Tauberian theorems. Oxford Univ. Press. [Sec 9, 10, 11] MR 21:5109

Pleijel, Å.

[1963] On a theorem of P. Malliavin. Israel J. Math. 1, 166-168. [Sec 1, 16, 19] MR 29:5023

Postnikov, A. G.

[1953] A Tauberian theorem for Dirichlet series (Russian). Doklady Akad. Nauk SSSR (N.S.) 92, 487-490. [Sec 1, 16, 18] MR 15:951b

[1979] Tauberian theory and its applications (Russian). Trudy Mat. Inst. Steklov 144, 147 pp. English transl.: Proc. Steklov Inst. Math. 1980, no. 2. [Sec 1, 12, 16, 17, 18] MR 82f: $40012 b$

Raikov, D.

[1938] Generalization of the Ikehara-Landau theorem (Russian). Mat. Sbornik. Recueil math. Akad. Nauk SSSR, N.S. 3, 559-568. [Sec 4]

Ransford, T. J.

[1988] Some quantitative Tauberian theorems for power series. Bull. London Math. Soc. 20, 37-44. [Sec 13] MR 89g:30005

Riemann, B.

[1892] Gesammelte mathematische Werke und wissenschaftlicher Nachlass (German). Teubner, Leipzig. (Reprinted by Dover Publ., New York, 1953.) [Sec 12]

Riesz, M.

[1909] Sur les séries de Dirichlet et les séries entières. C. R. Acad. Sci. Paris 149, 909-912. $[\operatorname{Sec} 1,12]$

[1911] Über einen Satz des Herrn Fatou. J. reine u. angew. Math. 140, 89-99. [Sec 1, 12]

[1916] Ein Konvergenzsatz für Dirichletsche Reihen. Acta Math. 40, 349-361. [Sec 1, 12]

[1924] Über die Summierbarkeit durch typische Mittel. Acta Litt. ac Scient. Univ. Hung. 2, 18-31. [Sec 12]

Selberg, A.

[1949] An elementary proof of the prime-number theorem. Ann. of Math. (2) 50, 305-313. [Sec 1] MR 10:595b

[1991] Collected papers, vol. 2. Springer, Berlin. [Sec 5] MR 95g:01032

Seneta, E.

[1976] Regularly varying functions. Lecture Notes in Math., vol. 508, Springer, Berlin. [Sec 17] MR 56:12189 
Subhankulov, M. A.

[1960] Tauberian theorems with remainder term. Mat. Sbornik 52 (94), 823-846; Amer. Math. Soc. Transl. (2) 26 (1963), 311-338. [Sec 1, 16, 18] MR 22:12334, MR 27:1745]

[1964] On a theorem of Littlewood (Russian). Izv. Akad. Nauk UzSSR, Ser. Fiz.-Mat. Nauk 1, 22-30. [Sec 1, 18] MR 29:5025]

[1973] Tauberian theorems of Landau-Ikehara type and their connection with the distribution of primes (Russian). Baškir. Gos. Učen. Zap. 74, 47-69. [Sec 4] MR 57:253]

[1976] Tauberian theorems with remainder (Russian). Izdat. Nauka, Moscow. [Sec 1, 4, 16, 18, 19] MR 58:29565]

Subhankulov, M. A. and An, F. I.

[1974] Complex Tauberian theorems for the one-sided and two-sided Stieltjes transform (Russian). Izv. Akad. Nauk SSSR Ser. Mat. 38, 138-169. (English translation: Math. USSRIzv. 8 (1975), 145-176.) [Sec 19] MR 51:6209]

Sz.-Nagy, B. and Foiaş, C.

[1970] Harmonic analysis of operators on Hilbert space. North-Holland Publ. Co., Amsterdam. [Sec 15] MR 43:947

Tauber, A.

[1897] Ein Satz aus der Theorie der unendlichen Reihen. Monatshefte Math. u. Phys. 8, 273277. [Sec 1]

Tenenbaum, G.

[1995] Introduction to analytic and probabilistic number theory. Cambridge Univ. Press. [Sec 4] MR 97e:11005b

Tenenbaum, G. and Mendès France, M.

[2000] The prime numbers and their distribution. Student Math. Library vol. 6, Amer. Math. Soc., Providence, R.I. (Translation of French edition of 1997.) [Sec 1] MR 2001j:11086

Titchmarsh, E. C.

[1927] On integral functions with real negative zeros. Proc. London Math. Soc. (2) 26, 185-200. [Sec 19]

[1939] The theory of functions. Oxford Univ. Press. (Second edition.) [Sec 12]

[1958] Eigenfunction expansions associated with second-order differential equations, Part II. Clarendon Press, Oxford. [Sec 19] MR 20:1065

Vaaler, J. D.

[1985] Some extremal functions in Fourier analysis. Bull. Amer. Math. Soc. (N.S.) 12, 183-216. [Sec 5] MR 86g:42005

Valiron, G.

[1914] Sur les fonctions entières d'ordre fini et d'ordre nul, et en particulier les fonctions à correspondance régulière. Ann. Toulouse (3) 5, 117-257. [Sec 19]

de la Vallée Poussin, C.

[1896] Recherches analytiques sur la théorie des nombres premiers, I. Ann. Soc. Sci. Bruxelles 20:2, 183-256. [Sec 1]

Vladimirov, V. S., Drozhzhinov, Yu. N. and Zav'yalov, B. I.

[1986] Multidimensional Tauberian theorems for generalized functions (Russian). Nauka, Moscow, 1986. (English translation: Tauberian theorems for generalized functions. Kluwer, Dordrecht, 1988.) [Sec 1] MR 87m:46092, MR 89j:46043

Widder, D. V.

[1941] The Laplace transform. Princeton Univ. Press, Princeton. [Sec 4] MR 3:232d

Wiener, $\mathrm{N}$.

[1928] A new method in Tauberian theorems. J. Math. and Phys. M.I.T. 7, 161-184. [Sec 1, 4]

[1932] Tauberian theorems. Ann. of Math. 33, 1-100. [Sec 1, 4]

[1933] The Fourier integral and certain of its applications. Cambridge Univ. Press, Cambridge. [Sec 1, 4]

Wiener, N. and Pitt, H. R.

[1939] A generalization of Ikehara's theorem. J. Math. and Phys. M.I.T. 17 (1939), 247-258. $[\operatorname{Sec} 4,9]$

Young, W. H.

[1918] On restricted Fourier series and the convergence of power series. Proc. London Math. Soc. 17, 353-366. [Sec 12] 
Zagier, D.

[1997] Newman's short proof of the prime number theorem. Amer. Math. Monthly 104, 705708. [1, 6, 7] MR 98j:11069

Zeller, K. and Beekmann, W.

[1970] Theorie der Limitierungsverfahren. Ergebn. Math. u. Grenzgebiete vol. 15, Springer, Berlin. (First edition 1958.) [Sec 12] MR 41:8863

Zygmund, A.

[1959] Trigonometric series. Cambridge Univ. Press. (Second edition.) [Sec 12] MR 21:6498

Department of Mathematics, University of Amsterdam, Plantage Muidergracht 24, 1018 TV AMsterdam, Netherlands

E-mail address: korevaar@science.uva.nl 